\title{
Estimation of the Real Area of Contact in Sliding Systems Using Thermal Measurements
}

\author{
William C. Schneck, III \\ Thesis submitted to the faculty of the \\ Virginia Polytechnic Institute and State University \\ in partial fulfillment of the requirements for the degree of \\ Master of Science \\ In \\ Mechanical Engineering
}

$\underline{\text { Committee Members }}$

Brian Vick, Chair

Thomas Diller

Scott Huxtable

23 September, 2009

Blacksburg, VA

Key Words: real area of contact, tribology, frictional heating, sliding surfaces, parameter estimation, cellular automata 


\title{
Estimation of the Real area of Contact in Sliding Systems Using Thermal Measurements
}

\author{
William C. Schneck, III
}

\begin{abstract}
This thesis seeks two objectives. One objective is to develop a means to estimate time invariant real contact areas and surface temperatures through thermal measurements in 1D/2D systems. This allows computationally easier models, resulting in faster simulations within acceptable convergence. The second objective is to provide experimental design guidance.

The methods used are a modified cellular automata technique for the direct model and a Levenberg-Marquardt parameter estimation technique to stabilize inverse solutions. The modified cellular automata technique enables each piece of physics to be solved independently over a short time step, thus frequently allowing analytical solutions to those pieces.

Overall, the method was successful. The major results indicate that appropriately selected measurement locations can determine the contact distribution accurately, and that the preferred measurement location of the sensor is not very sensitive to the contact distribution specifics. This is useful because it allows selection of measurement locations regardless of the specifics of the generally unknown contact distribution. Further results show the combined effects of the normalized length and the Stanton number have a significant impact on the estimation quality, and can change the acceptable sensor domain, if the loss is high. The effect of placing the sensor in the static body can, for low loss, provide a coarse image of the contact distribution. This is useful because the static body is easier to instrument than a moving body. Finally, the estimation method worked well for the most complex model utilized, even in a suboptimal measurement location.
\end{abstract}




\section{Acknowledgements}

First of all, I would like to extend my deepest thanks to my advisor, Dr. Brian Vick, for his efforts, patience, and support while working on this thesis. Without his assistance, this thesis would not be what it is today. Further, his mentorship has helped me not just with the technical aspects of being an engineer, but also with more esoteric topics regarding engineering pedagogy and philosophy.

Secondly, I would like to extend my thanks to the faculty of Virginia Tech, especially my committee members Dr. Huxtable and Dr. Diller, along with my future Ph.D. advisor Dr.

O'Brien, for having created a friendly and welcoming environment that was highly conducive to learning. I especially wish to thank all my professors from whom I have taken classes, and been able to interact with and learn from as a result.

I would also like to thank Dr. Linda Vick, for helping me with course planning and with finding Dr. Brian Vick when I was looking for him.

I also thank Dr. Kasarda, Dr. Stern, Dr. Huxtable, and Dr. Johnson for allowing me to serve as a teaching assistant for the ME 4005 and 4006 classes. The experiences gained while interacting with the students regarding these lab classes provided great lessons for me.

I thank the Virginia Space Grant Consortium and John Companion for providing the initial funding over the course of my first summer that got this project started.

I would like to say thanks to my friends Kat Tran, Tony Ferrar, Dan Villareal, and Steve Mitchell who have provided various bits of assistance on this thesis ranging from editing to formatting to technical discussion regarding the material. The assistance and fun that have gone along with working with them has helped keep the harder parts of this project from feeling insurmountable.

Outside of Virginia Tech, I would like to thank my various mentors. In particular, the guidance and confidence that I gained from them has enabled me to achieve what I have so far. Thanks to Dr. Brad Libbey, Jim Campbell, and Charles Amazeen from the S\&T Division at the Army's Night Vision Lab at my first Co-op job, and Steve Bennett, Tony Tomlinson, Stu Leigh, and Larry Jackson from the Countermine Division also at the Night Vision Lab at my second.

Also, I would like to extend my thanks to Rene Gonzalez, who was a great mentor of mine. He is an outstanding engineer, both in terms of the skills I have seen and in terms of the 
ethics with which he comports himself. He has helped me with many engineering problems I have faced.

My father, Bill, has also served as a role model for me as an engineer. He has always been supportive of my engineering endeavors, and has been willing to assist me in these technical matters at the drop of a hat. He has encouraged me to be the best engineer I can be and has done wonders for my confidence as a student of the natural sciences.

Lastly, I would like to thank my family, in particular my mother, Cathy, and sister, Katie. Without all of your support and tolerance of my academic pursuits, I would not be here today. I send you all my sincerest love and appreciation. 


\section{Table of Contents}

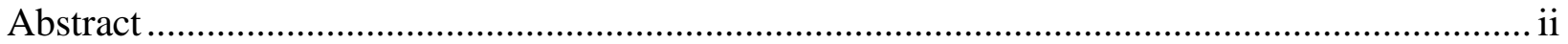

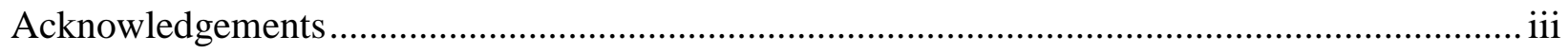

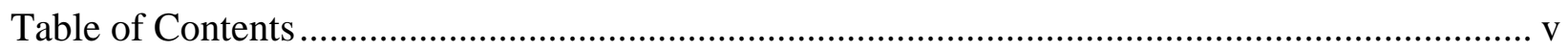

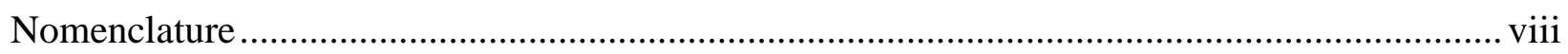

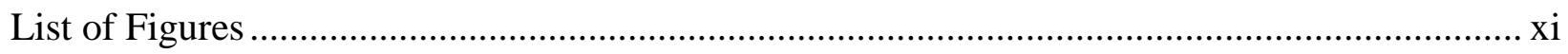

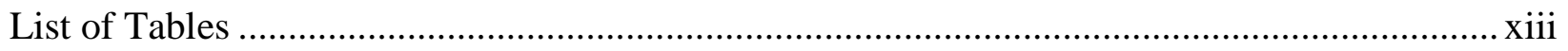

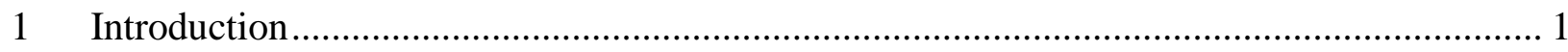

1.1 Motivation .................................................................................................. 1

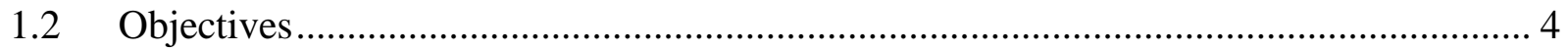

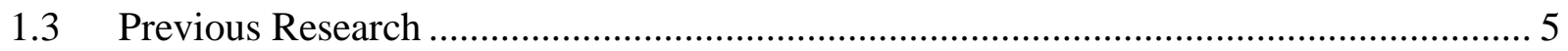

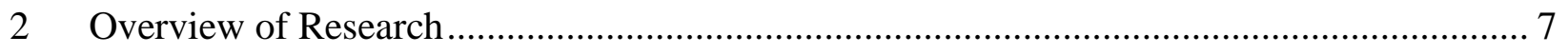

2.1 The Physical System ................................................................................. 7

2.2 Solution of the Direct Problem.............................................................................. 8

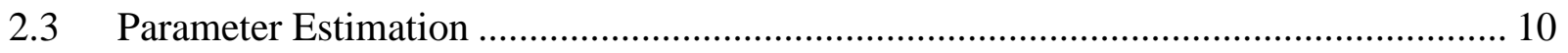

3 Physical Formulation of the Direct Model.............................................................. 12

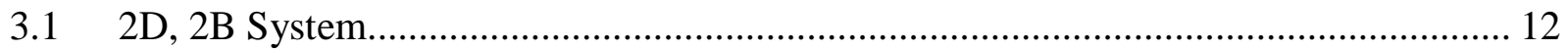

3.1.1 General Physical Schematic .................................................................. 12

3.1.2 General Contact Area Profile .......................................................................... 13

3.1.3 Formulation of the 2D, 2B Problem ......................................................... 14

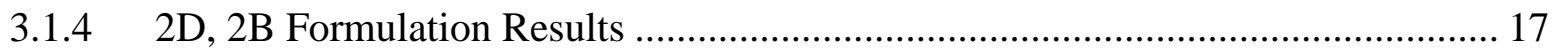

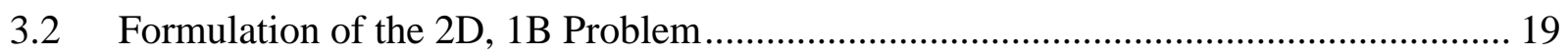

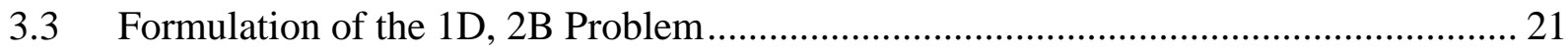

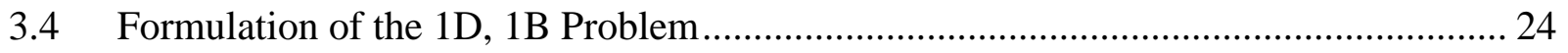

4 Solution of the Direct Problem via Modified Cellular Automata.................................... 26 


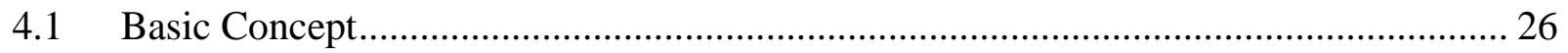

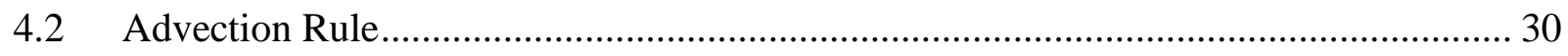

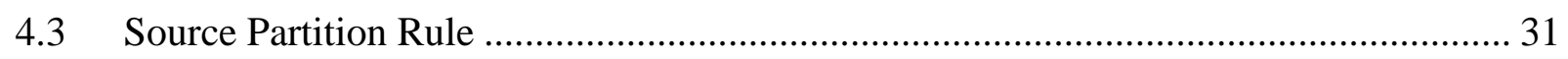

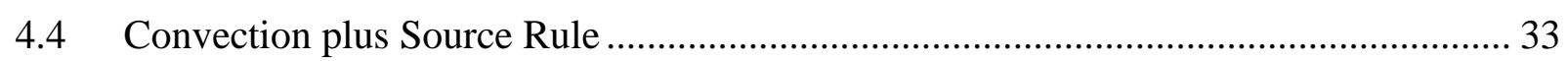

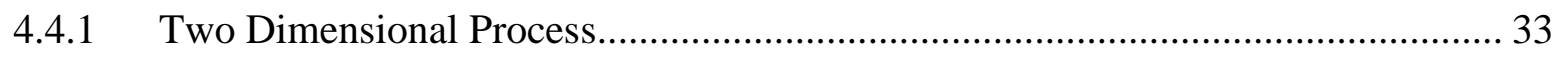

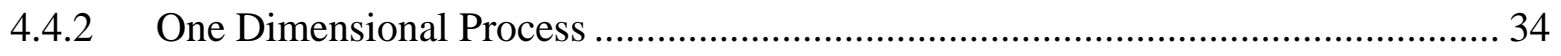

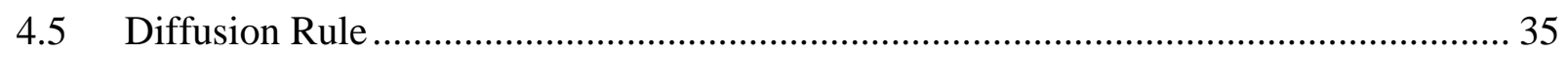

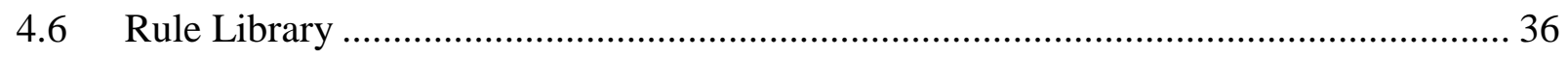

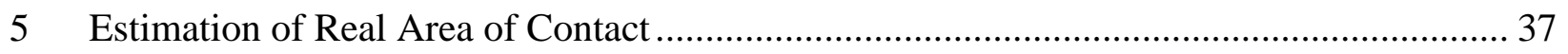

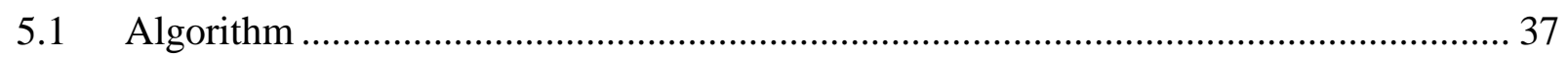

5.2 Application to Real Area of Contact......................................................................... 42

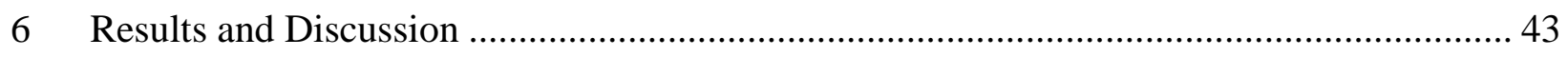

6.1 Non-Dimensionalization of the 2D, 2B Equations...................................................... 43

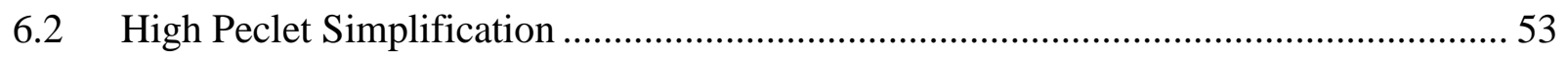

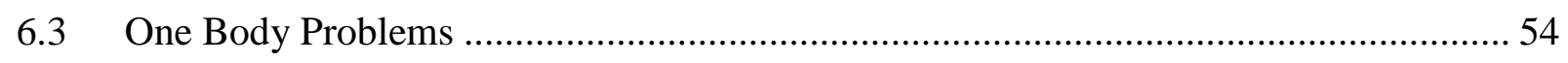

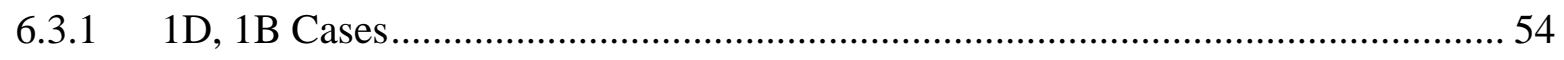

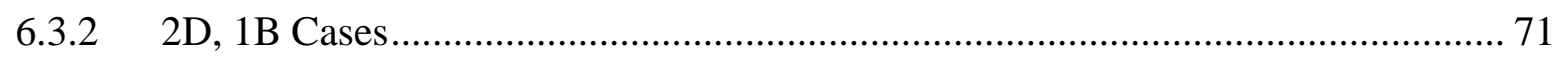

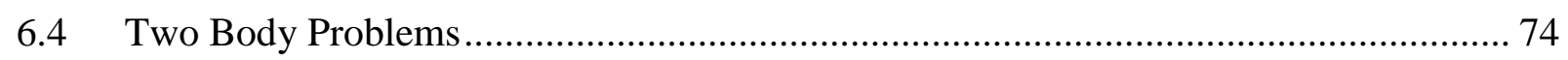

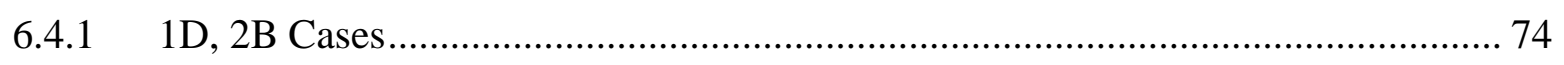

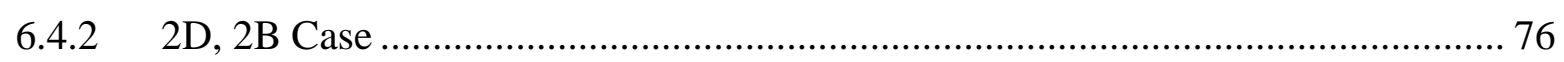

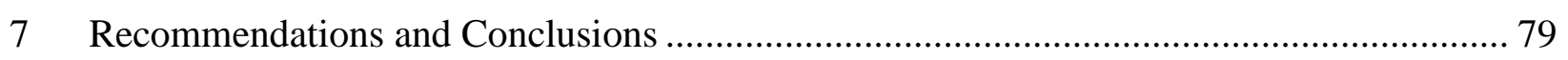

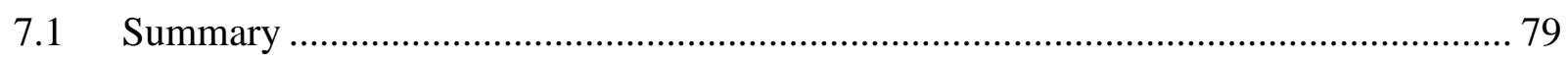

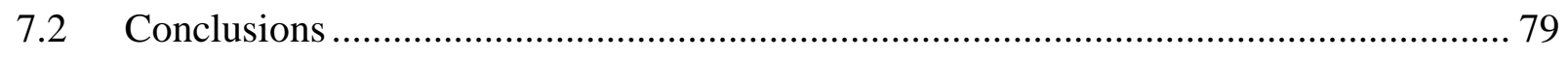

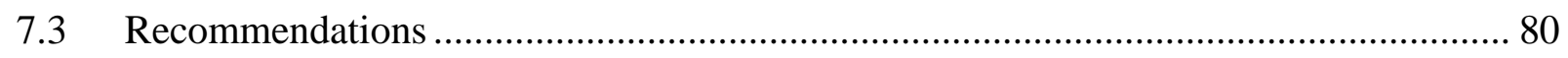

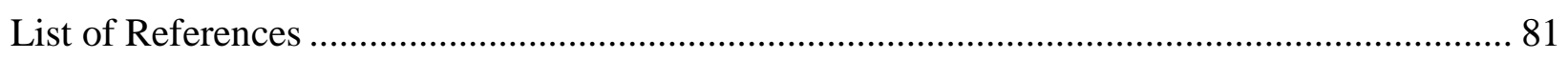


Appendix 


\section{Nomenclature}

\section{English}

$\boldsymbol{a}_{u}=$ upper diagonal

$\boldsymbol{a}_{d}=$ major diagonal

$\boldsymbol{a}_{l}=$ lower diagonal

$\boldsymbol{A}=$ tridiagonal matrix

$A_{c}=$ cross sectional area $\left(\mathrm{m}^{2}\right)$

$c=$ heat capacity of solid $\left(\frac{\mathrm{J}}{\mathrm{kg} \cdot \mathrm{K}}\right)$

err $=$ magnitude of error imposed on simulated data $(K)$

$f_{d}(x, t)=$ unit distribution of heat due to friction $\left(\frac{1}{m}\right)$

$\Delta f_{d_{R M S}}=$ contact distribution residual $\left(\frac{1}{m^{2}}\right)$

$\boldsymbol{f}(\quad)=$ process in modified cellular automata method $\left(\frac{K}{S}\right)$

$F_{n}=$ normal force applied to contact $(N)$

$\mathcal{F}(\quad)=$ rule in modified cellular automata method $(K)$

$h=$ convective loss (to environment) coefficient $\left(\frac{W}{m^{2} \cdot K}\right)$

$H(\quad)=$ heaviside step function

$I=$ identity matrix

$k=$ thermal conductivity $\left(\frac{W}{m \cdot K}\right)$

$L=$ length $(m)$

$N=$ an integer

$P=$ perimeter of cross section of solid $(m)$

$P e=$ Peclet number, $\frac{V L_{\text {char }}}{\alpha}$

$q=$ total heat evolved due to a process $(W)$

$q^{\prime}(x, t)=$ linear heat flux distribution due to a process $\left(\frac{W}{m}\right)$

$q^{\prime \prime}(x, t)=$ area heat flux distribution due to a process $\left(\frac{W}{m^{2}}\right)$

$\boldsymbol{R}=$ residuals vector

$S=$ sum of squares of residuals

$S_{c}=$ general source term independent of temperature $(W)$

$S t=$ Stanton number,$\frac{h}{\rho c V}$

$t=$ time $(s)$ 
tridiagonal $(\quad)=$ tridiagonal solver method

$\Delta t=$ time step for the modified cellular automata method $(s)$

$T=$ temperature $(K)$

$V=$ velocity of solid $\left(\frac{m}{s}\right)$

$x=$ coordinate in $x-$ direction $(m)$

$\Delta x=$ variable used in $C V$ analysis or node spacing in numerical solution ( $m$ )

$X=$ sensitivity coefficient

$\boldsymbol{X}=$ sensitivity matrix

$y=$ coordinate in $y-$ direction $(m)$

$\Delta y=$ variable used in $C V$ analysis or node spacing in numerical solution ( $m$ )

$Y=$ measurement

$\boldsymbol{Y}=$ vector of measurements

\section{Greek}

$\alpha=$ thermal diffusivity $\left(\frac{m^{2}}{s}\right)$

$\beta=$ parameter

$\boldsymbol{\beta}=$ parameters vector

$\theta=$ temperature rise above ambient $(K)$

$\mu=$ damping parameter for Levenberg - Marquardt Method

$\mu_{k}=$ sliding coefficient of friction (unitless)

$\xi=$ index in talyor series

$\Xi=$ terminal index in taylor series

$\rho=$ density of solid $\left(\frac{\mathrm{kg}}{\mathrm{m}^{3}}\right)$

$\tau=$ time constant variable for substitution $(s)$

\section{Super/subscripts}

$$
\begin{aligned}
& \begin{array}{l}
a \\
b
\end{array}=\text { exponent on } V \text { to achieve a characteristic value } \\
& { }^{c}=\text { exponent on } \frac{q_{\text {fric }}}{\rho_{2} c_{2} L_{y_{2}} L_{z} L_{c o n}} \text { to achieve a characteristic value } \\
& { }^{T}=\text { transpose } \\
& { }^{+}=\text {dimensionless } \\
& \text { adv }=\text { pertains to advection rule } \\
& { }_{b o d y}=\text { pertains to one of the two modeled bodies }
\end{aligned}
$$




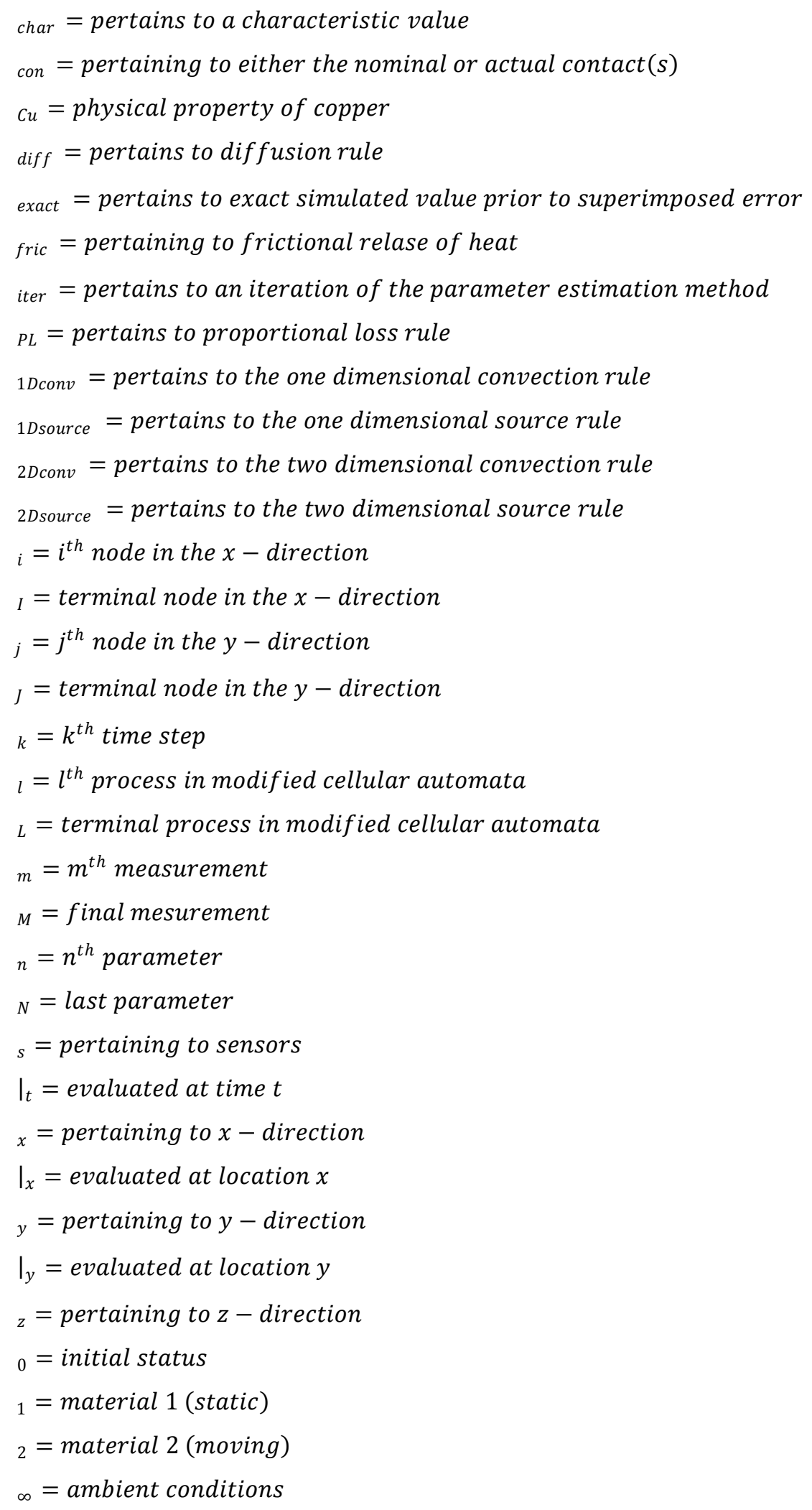




\section{List of Figures}

Figure 1.1: Analytical result of temperature rise over real contact area [1] ............................. 2

Figure 1.2: Sample picture of an actual contact [2] .......................................................... 3

Figure 1.3: Silicon wear scar showing a localized contact [2] ........................................... 4

Figure 2.1: The parameter estimation process. ................................................................ 7

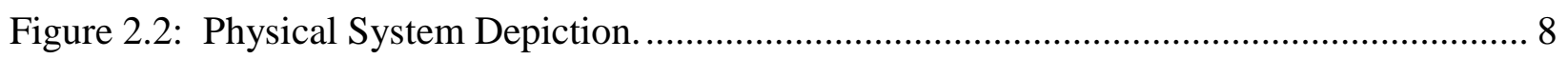

Figure 2.3: Schematic representation of the direct problem.................................................. 9

Figure 2.4: Pictorial representation of the modified cellular automata method. ........................ 10

Figure 2.5: Schematic representation of the estimation of the forcing function........................ 11

Figure 2.6: Schematic representation of the estimation of system parameters.......................... 11

Figure 3.1: Schematic portrayal of the physical system in Figure 2.2 .................................. 12

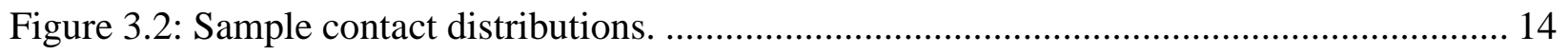

Figure 3.3: Geometric layout of the heat transfer equations by control volume analysis. .......... 15

Figure 3.4: 2D, 2B formulation summarized by physical location.......................................... 18

Figure 3.5: 2D, 1B formulation summarized by physical location....................................... 20

Figure 3.6: 1D, 2B formulation summarized by physical location........................................ 23

Figure 3.7: 1D, 1B formulation summarized by physical location......................................... 24

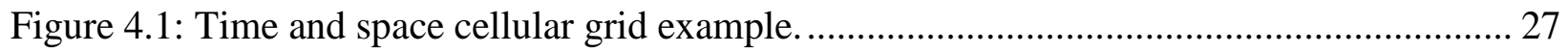

Figure 4.2: Graphical representation of the modified cellular automata feed-through............... 29

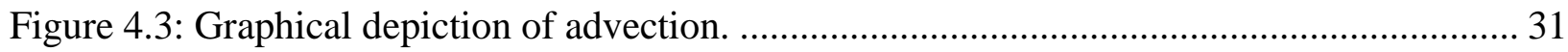

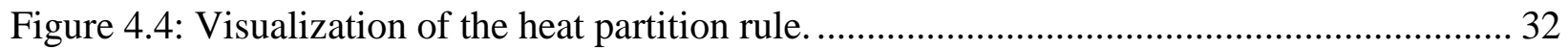

Figure 5.1: Depiction of knowns and unknowns in the real area of contact estimation problem. 37

Figure 5.2: Conceptual layout of measurement locations................................................ 37

Figure 5.3: Conceptual representation of one and two parameter sums of squares................... 38

Figure 5.4: Parameter estimation algorithm flowchart. ................................................... 41

Figure 5.5: Arbitrary contact distribution discretized for estimation. ................................... 42

Figure 6.1: Bulk visualization for determining physical length scale. .................................. 53

Figure 6.2: Temperature fields for 100 and 200 node simulations...................................... 55

Figure 6.3: Sample dimensionless temperature data taken at a normalized location of $x_{s}^{+}=1.5 .56$

Figure 6.4: Dimensionless contact distribution comparison............................................. 57

Figure 6.5: Dimensionless data simulated at 1.1 and 6.1 contact lengths. ............................... 58 
Figure 6.6: Estimated dimensionless contact distributions for various input distributions.... 59

Figure 6.7: Selected sensitivity coefficients at measurement locations of 1.1 and $6.1 \ldots \ldots \ldots \ldots . . . .60$

Figure 6.8: Contact distribution RMS residuals, with varying contact distributions. ................ 61

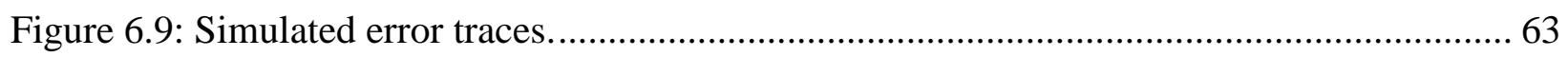

Figure 6.10: Contact distribution RMS residual for varied error case.................................... 63

Figure 6.11: Contact distribution residual for the total lengths of $L_{x}{ }^{+}=10,5,2 \ldots \ldots \ldots \ldots \ldots \ldots \ldots \ldots . .65$

Figure 6.12: Contact distribution RMS residual for various dimensionless lengths. ................ 67

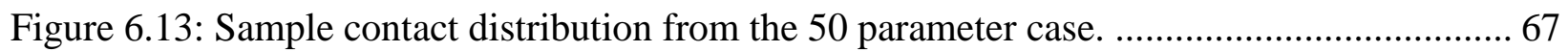

Figure 6.14: Contact distribution RMS residual density plot. ............................................. 69

Figure 6.15: Contact distribution RMS residual plots for various sensor locations................... 70

Figure 6.16: Comparison of dimensionless temperature using 100 and 200 ' $\mathrm{y}$ ' nodes............... 72

Figure 6.17: Contact distribution RMS residual plot for one sensor in two dimensions............. 73

Figure 6.18: Contact distribution RMS residual plot for two bodies with geometry shown....... 75

Figure 6.19: Sensitivity coefficients for each body at their preferred location. ....................... 76

Figure 6.20: Actual and estimated contact distributions for the 2D, 2B demonstration.............. 78 


\section{List of Tables}

Table 3.1: 2D, 2B formulation summarized in tabular form. .................................................. 19

Table 3.2: 2D, 1B formulation summarized in tabular form. ................................................... 21

Table 3.3: 1D, 2B formulation summarized in tabular form. ..................................................... 24

Table 3.4: 1D, 1B formulation summarized in tabular form................................................... 25

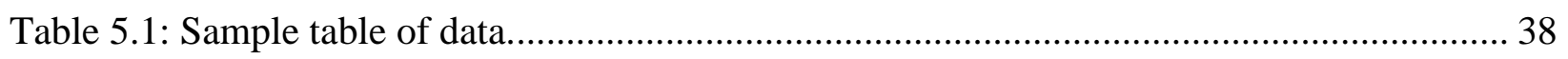

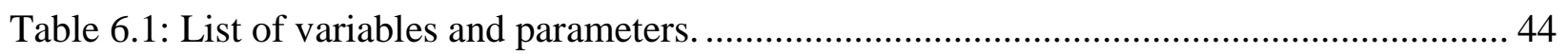

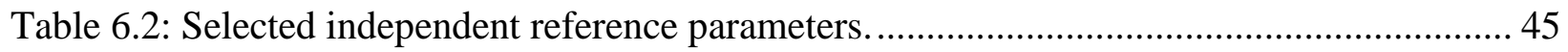

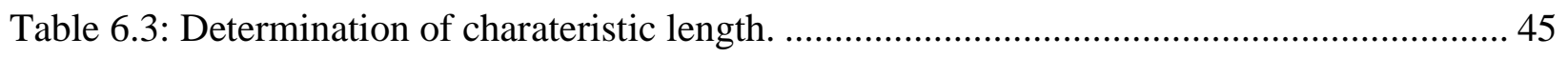

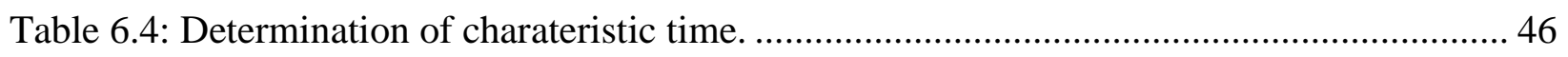

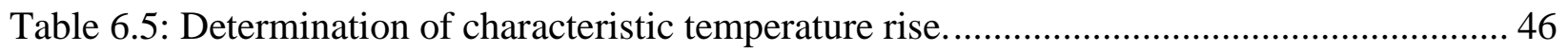

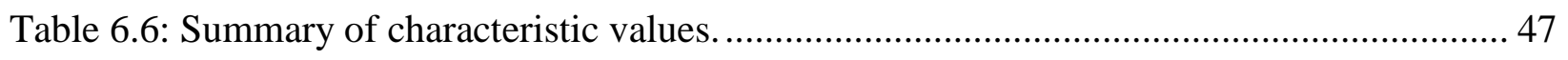

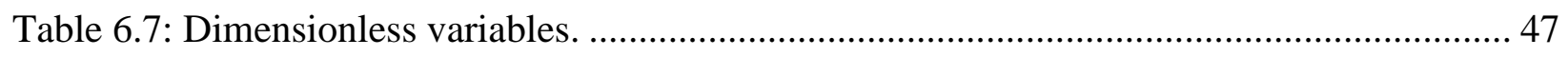

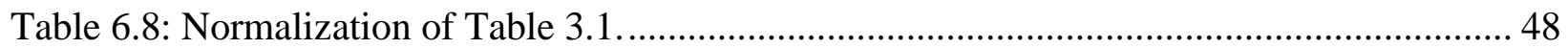

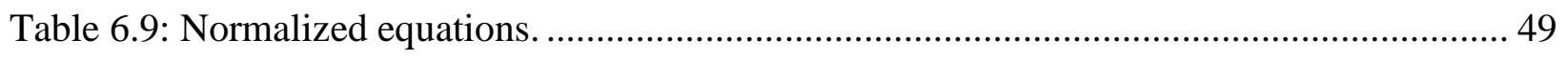

Table 6.10: Normalized 2D, 2B equations with dimensionless parameters. ............................... 50

Table 6.11: Normalized 2D, 1B equations with dimensionless parameters.................................. 51

Table 6.12: Normalized 1D, 2B equations with dimensionless parameters. ................................ 52

Table 6.13: Normalized 1D, 1B equations with dimensionless parameters................................. 52

Table 6.14: Physical parameters for the numerical resolution case................................................ 54

Table 6.15: Parameter values for sample case ..................................................................... 56

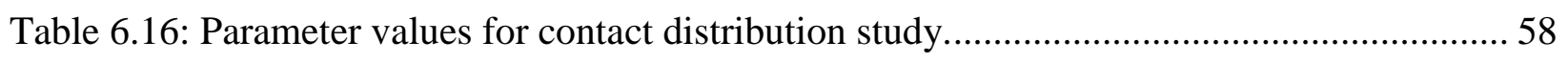

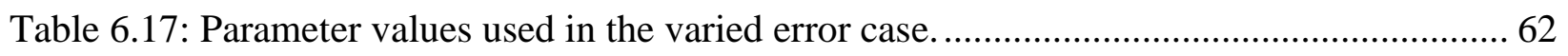

Table 6.18: Parameter values used in the total length study....................................................... 64

Table 6.19: Parameter values used in the contact length study. .................................................... 66

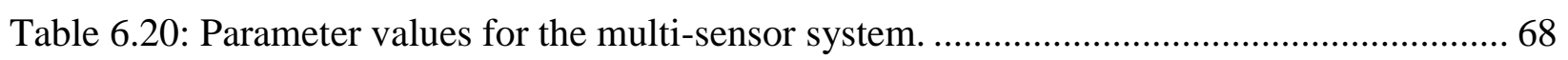

Table 6.21: Physical parameters for the 2D numerical resolution study...................................... 71

Table 6.22: Parameters for the varied 'y' measurement location. ................................................. 73

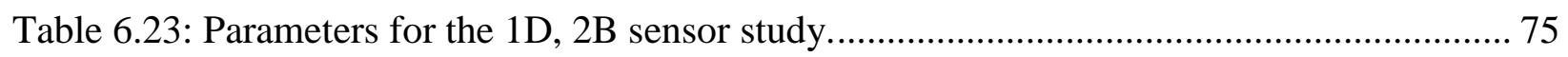

Table 6.24: Parameters for the demonstration of the 2D, 2B estimation....................................... 77 


\section{Introduction}

This introduction will briefly cover the motivations for the work presented, the major objectives, and the previous work done in the applicable fields.

\subsection{Motivation}

Sliding systems occur throughout industrial and natural processes. The resultant frictional heating and mechanical loads are a major driver of expensive maintenance costs. Brakes, bearings, and other rolling/rotating systems all exhibit this sliding behavior. Outside of rotating machinery, other sliding systems such as certain machining processes, internal ballistics in guns, and guide rails (such as in an optical disc drive). In the natural world, biotribology includes the rubbing of joints. In other words, tribological processes occur everywhere there is relative motion. In each of these systems, components (such as tooling) have demonstrated that they eventually wear out under their operating conditions. Thus, in the course of one's observations of these systems, one must be able to detect the near onset of failure. The closer one can get (reliably, and without actual failure), the more economical the system. One of the key steps, therefore, is to know how to effectively instrument these systems.

The physical effects at sliding contacts involve a complicated interaction of thermal, mechanical, and chemical processes. The real area of contact inside the nominal contact zone is of critical importance to the understanding these complete processes, particularly the temperature behavior of the system. As the real contact area is reduced, the surface temperature increases significantly. This increase is because there is less material under the contact that must absorb the same amount of energy. Indeed, if one has a significant uncertainty in the knowledge of the real contact area, estimating the surface temperature rise is effectively impossible. This relationship is demonstrated in Figure 1.1 where the average contact temperature rise is shown versus assumed contact area for some selected metals [1]. 


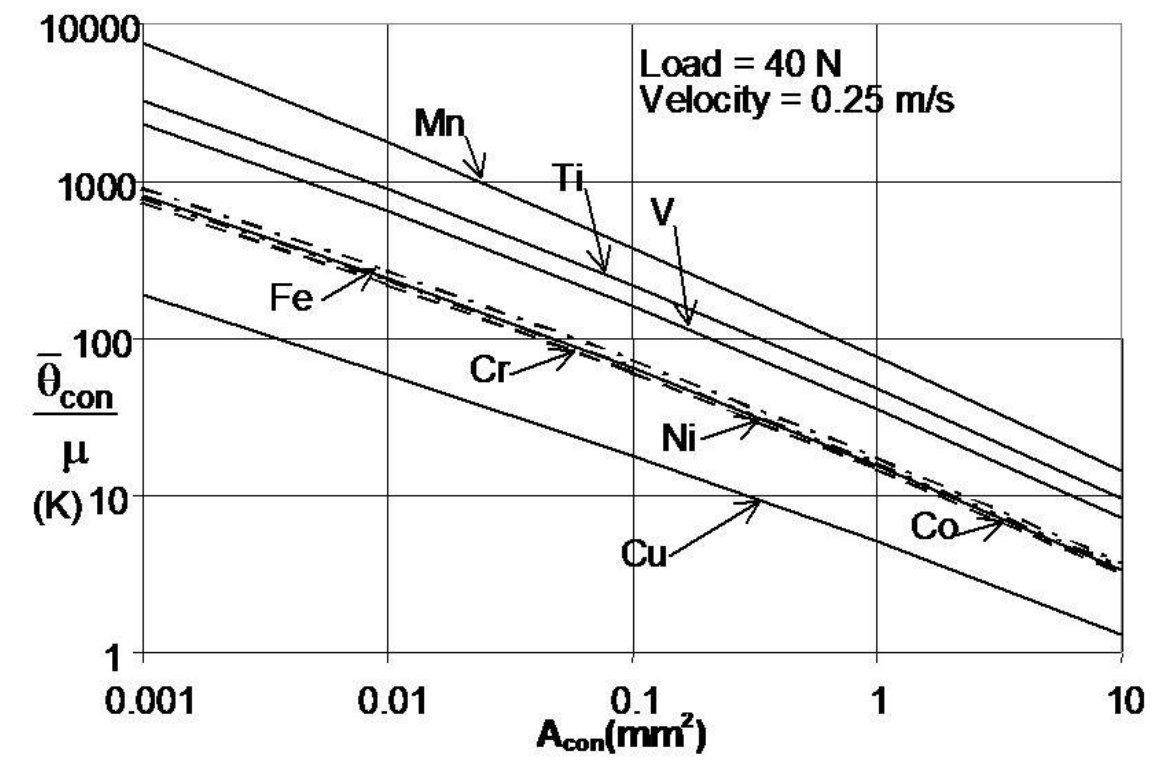

Figure 1.1: Analytical result of temperature rise over real contact area [1].

The temperature rise is expressed as the change in temperature divided by the friction coefficient.

Notable about these trends is the large orders of magnitude, shown on the log-log plot. As displayed in figure, without knowledge of the real area of contact, the order of magnitude of the temperature rise cannot even be estimated. Because the temperature of a material significantly influences its chemistry and certain physical properties, knowledge of the peak temperatures is important. Overheating of the material as a result of frictional interaction can result in mechanical failure of the part, or excursion of the mechanical properties from the anticipated design properties. These effects can render the part useless. Because the real contact area strongly affects the peak temperatures, the real area of contact is extremely important. Some of the consequences of high temperatures caused by frictional heating are tribochemistry, chemical oxidation, wear, thermal expansion, thermal cracking, melting, work hardening, and property changes.

This contact area depends greatly on many things. Chemistry and solid mechanics are of primary importance. These interrelationships are very complex and difficult to characterize. These interrelationships result in distinct modeling difficulties. Further, these contacts can be rather fractal-like. This fractal-like appearance makes determining the real contact area very complex, because as one looks closer at each contact, more and more area is seen to be in contact. This behavior can be seen in Figure 1.2 [2]. This particular image was taken in conjunction with experiments run for [3]. This image depicts a polystyrene coated steel ball 
fretting against sapphire optical flats. The sapphire enables one to look through it in the infra-red spectrum and directly observe the contacts. The imaging capabilities could resolve down to the 10 micron scale.

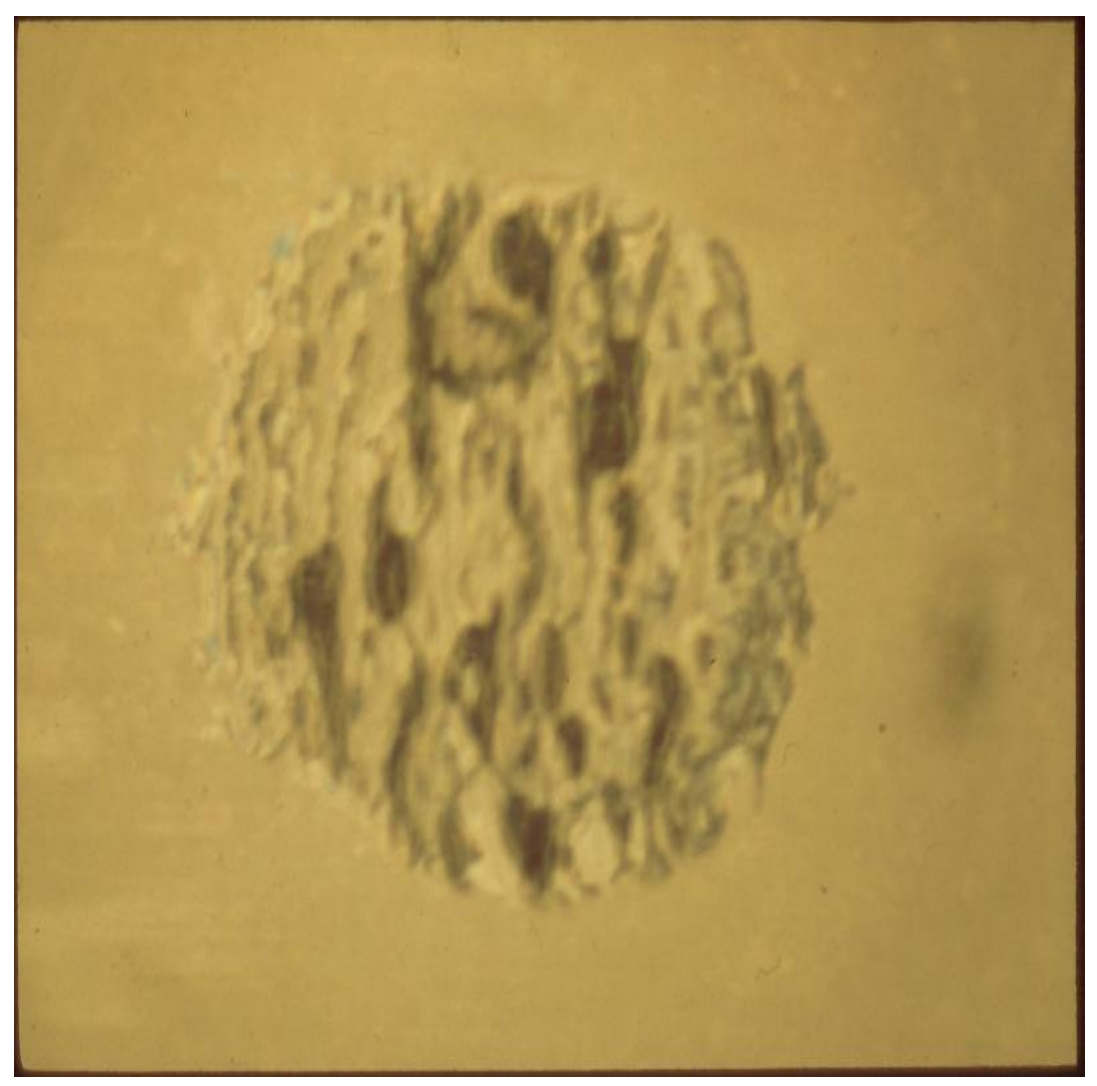

Figure 1.2: Sample picture of an actual contact [2].

It is, however, frequently possible to determine a nominal contact area through various means. One can tell, by looking at the above picture, that the contact is limited to the roughly circular region where all of the dark patches are located. Many times, however, the nominal contact area is determined by observing wear scars after the experiment. This determination is frequently done using scanning electron microscopy. An example of this method is provided below in Figure 1.3. This picture is an image of a similar pin-on-disk system with a silicon wear scar created by a diamond pin. 


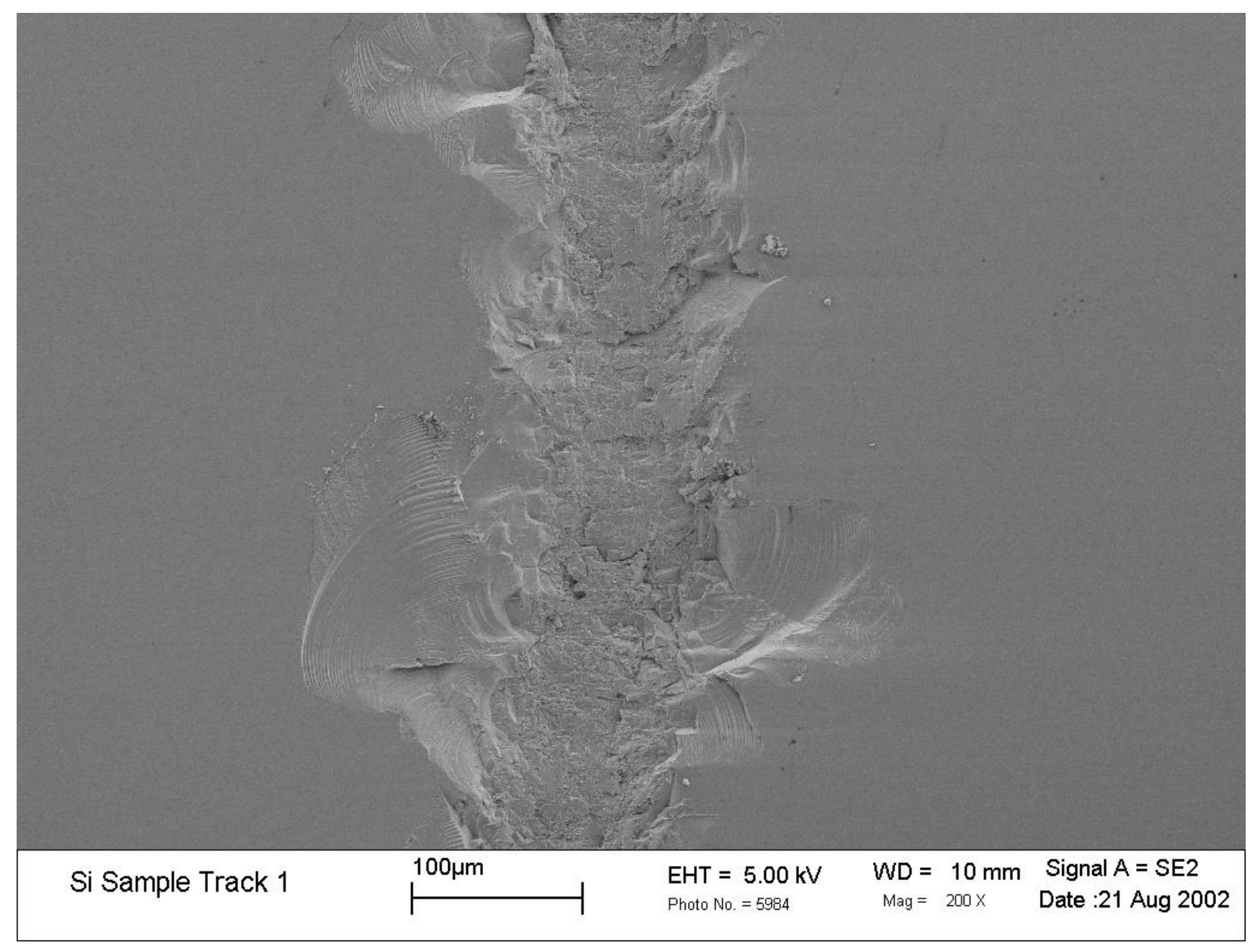

Figure 1.3: Silicon wear scar showing a localized contact [2].

Finally, in the majority of systems, since most materials are opaque to available instrumentation, it is impossible to directly observe the contact. That, combined with the above two issues of importance and complexity, make the determination of the real area of contact a very difficult, but critical, field of study.

\subsection{Objectives}

The objectives of this thesis are as follows:

- To estimate a time invariant real area of contact and surface temperature through the use of thermal measurements in 1D and 2D systems.

- To provide experimental design guidance regarding measurement locations for the estimation of the real contact area. 


\subsection{Previous Research}

Much previous work has been done regarding parameter estimation, heat transfer, and real contact area. However, most of the literature regarding the real contact area involves direct modeling using more statistically generated surfaces and surface mechanics, or attempting to understand something about it using analysis of scarring. The literature that does regard estimating the real contact area uses several different means of estimation. The most frequent method seemed to be function estimation.

Much of the tribological literature either uses very simplified models of, or experimentally estimates, contact area as a means to get at very important topics such as flash temperature (the peak temperature at the interface between the two bodies). Flash temperature is important because, at elevated temperatures, certain mechanisms for surface chemistry can be activated that can cause damage to the physical system. This research is of interest as it shows the importance of flash temperature, and its dependence on contact area. The works which cover this material include [1, 3-14].

The analysis using surface mechanics involves direct modeling using an initial surface based on some measurements of real surfaces or simply measuring the time dependent contact area [3]. It then evolves the contact through time allowing for deformations and other assorted physical phenomena to act. The measurements of the real surfaces can take the form of scanning electron microscopy, average surface roughness measurements, and the like. The measurement of the real contact area has been done using scanning infrared microscopy through a sapphire disk (because of sapphire's transparency in the IR). The works that approach this discussion in this manner are [3, 9, 15-22].

Function estimation techniques assume a (frequently polynomial) function where the parameters of the function are estimated. This approach implies, for instance, if the contact were approximated to be quadratic, there would be 3 parameters to estimate, with there being only a single contact. However, other means are utilized. The primary drawback to almost all the literature regarding the estimation is that they study a very concrete example with little in terms of generalized results.

Of particular interest in this category were works [23] and [24]. These particular works studied three different means of function estimation for the heat flux distribution in a grinding 
process. These techniques are not suitable for allowing a random contact distribution, but provide useful insight into a single particular type of system.

One work [25], does present the dimensionless equations, but because the work was focused on grinding processes, it only utilizes a finite rectangular prism, and thus lacks a study of periodic boundaries, as would be present in rotors. Further, it does not delve into a study of the behavior of the estimation technique under various parameter values. The paper also presented more of a function estimation scheme than a true parameter estimation technique.

Much of the available literature regarding parameter estimation deals with generic inverse heat conduction. Inverse heat conduction has been studied for quite some time, as it is a necessary capability in many applications. One such application is in propulsion engineering regarding the heat flux through the wall of rocket/jet engines [26]. However, of most interest to this thesis is the study of the stability and resolving capability of various inverse heat transfer techniques. The relevant works are [27-35]. Of particular interest is [27], as it provides very clear descriptions of multiple methods of parameter estimation. The descriptions are clear enough on the mathematics to enable one to rapidly implement the methods for whatever application.

The direct thermal model is the final aspect of the problem that requires discussion. The direct thermal model was derived using fundamental techniques. The formulation of the governing equations uses techniques presented in undergraduate heat transfer texts such as [36] and the course notes [37] for the graduate conduction class. After the full formulation, further techniques can be used to further simplify the model to reduce the computational load. Of particular interest is the high Peclet number simplification which, while repeated mathematically in this thesis, is further confirmed in [38]. This simplification is able to remove one direction of conduction, the finite difference solution of which is one of the big computational loads. Finally, in order to more easily develop numerical models for and solve the system as derived, a modified cellular automata technique was utilized. This technique prior to its modification is well demonstrated in [39]. 


\section{Overview of Research}

This research requires the development of mathematical models of the physics pertaining to the temperature fields evolved in sliding solids undergoing frictional heating in order to infer the real area of contact. The system, both mathematically and physically, can be seen below in Figure 2.1.

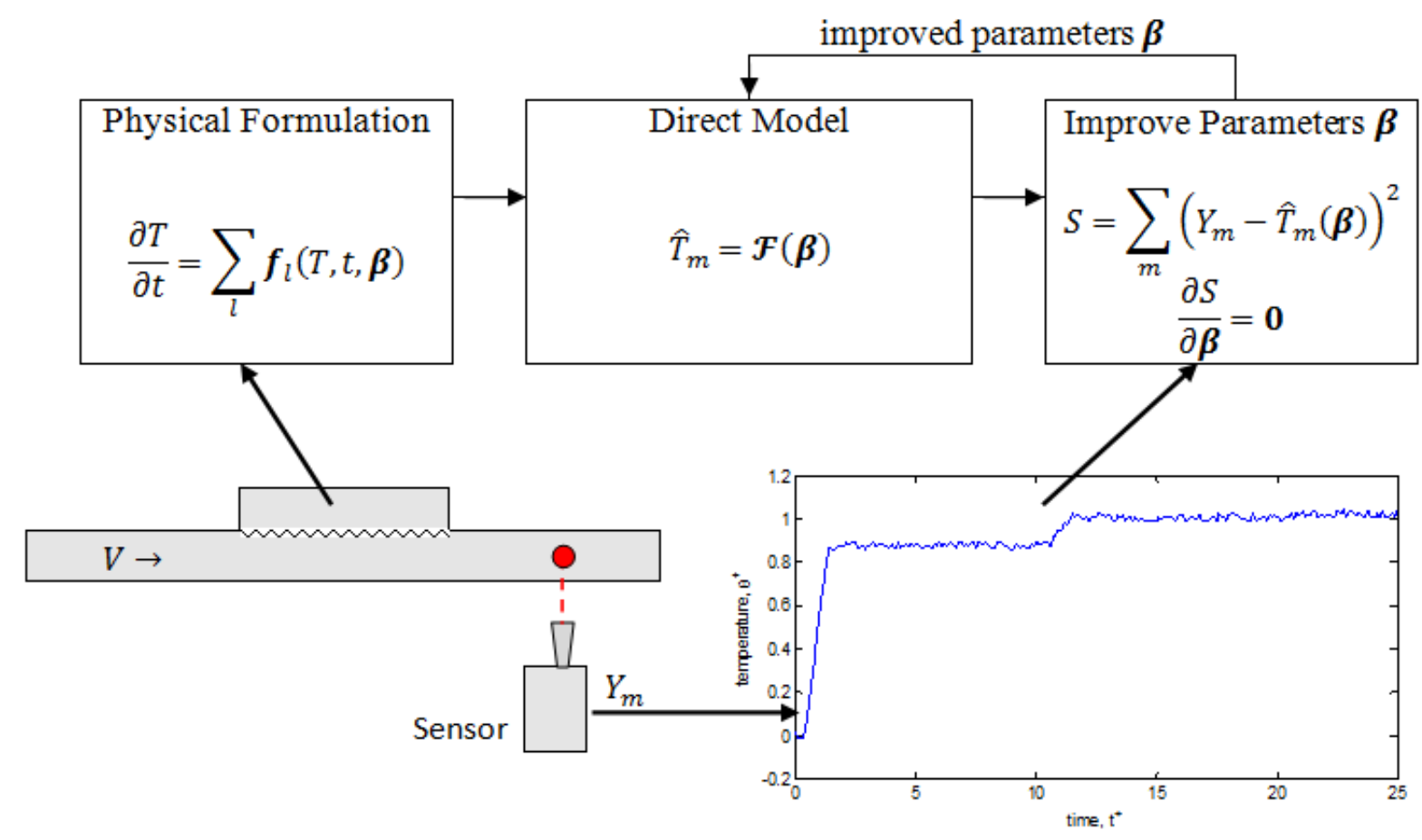

Figure 2.1: The parameter estimation process.

The physical system as it conceptually could be measured and how it relates to the math.

The model must start from a proposed physical system, and be evolved mathematically through a direct numerical model that is used in a parameter estimation scheme to approximate the contact distribution from which the real area of contact is inferred.

\subsection{The Physical System}

This paper deals with a two body system undergoing frictional heating. Figure 2.2 depicts a possible physical setup for this system, consisting of a light-colored rotating structure (2) that is rubbing against a dark-colored static pad (1) over a localized area. Braking type systems inspired this construct. The presumed direction of rotation is depicted in the figure. 


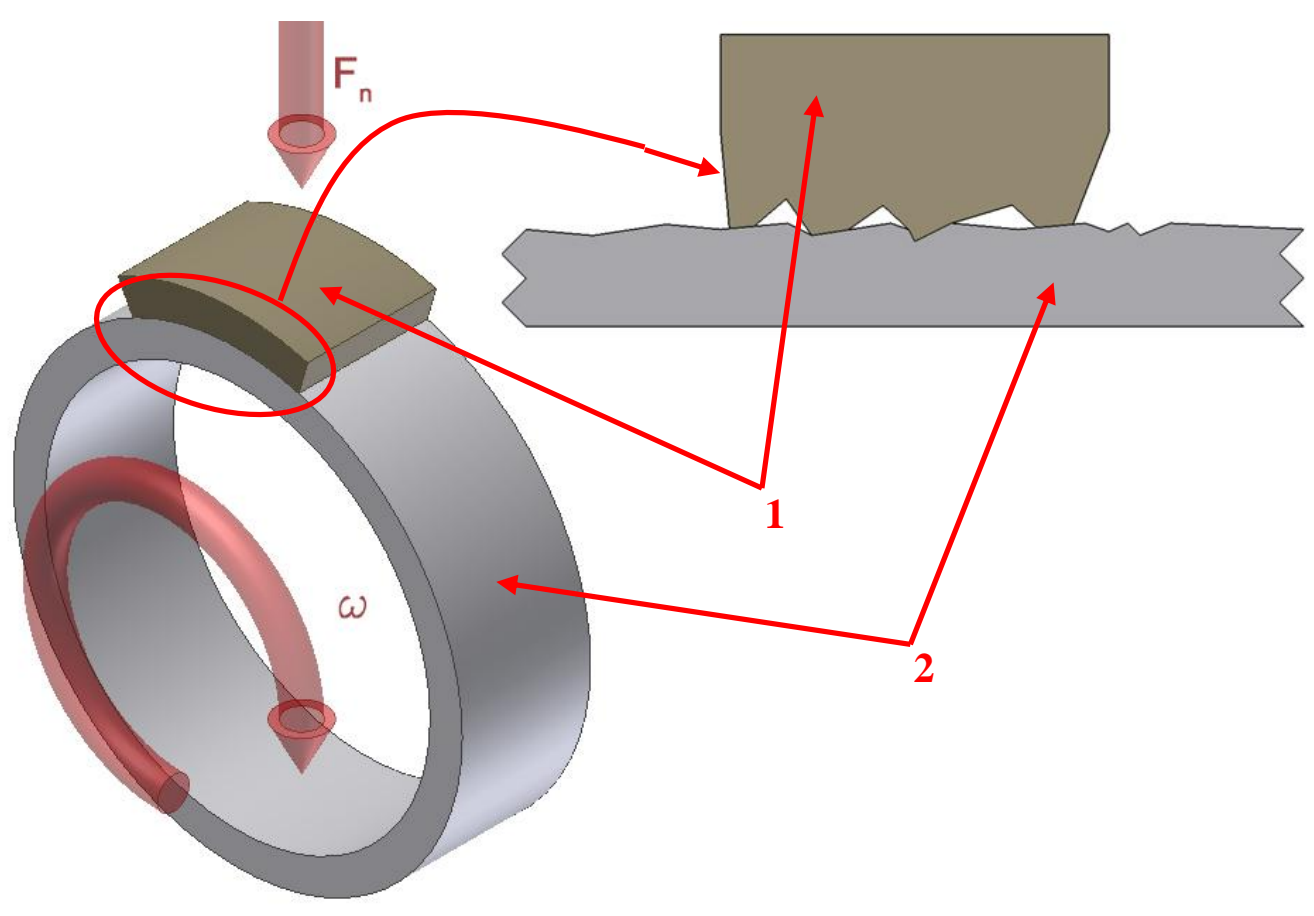

Figure 2.2: Physical System Depiction.

This system is well suited to wrapped boundaries, such that conditions at $x=0$ equal conditions at $x=L_{x}$. The inset is a detailed view of the interface.

The inset to Figure 2.2 shows an exaggerated, schematic close-up of the contact region. One can see the rough, uneven contact. Frictional heat generation will occur at the points where the two regions are actually in contact, and be dependent on the pressure of the contact and the velocity of the moving material.

\subsection{Solution of the Direct Problem}

Mathematically, the direct problem is the determination of the response of a system with known forcing functions and system parameters. This problem is represented in Figure 2.3. This is the classic type of problem found in most engineering analysis, where the system's excitation and characteristics are known, but the response is not. Mathematically, the direct problem is well-posed. This statement implies that a solution exists, is stable, and is unique. This statement further means that for any given forcing function, there is one and only one solution, and that the result will not become unstable under small changes to input data [27]. 


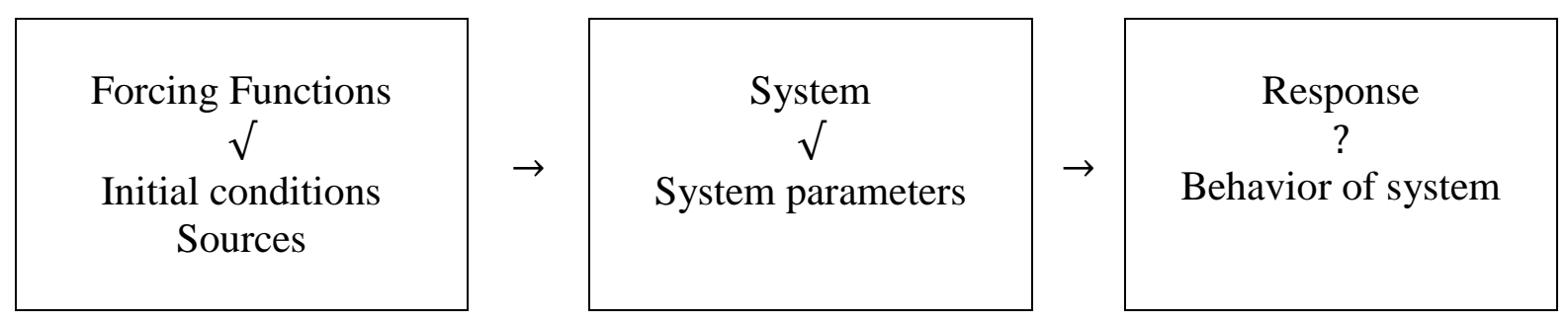

Figure 2.3: Schematic representation of the direct problem.

Mathematically, the physics surrounding the direct problem in this case are complex enough to make it impossible to achieve an analytical solution. Thus, one must choose from any number of numerical methods to arrive at a computational solution. In order to achieve a faster simulation that would allow the multiple iterations necessary to perform parameter estimation, a modified cellular automata method was chosen to handle the multi-physics.

\section{Modified Cellular Automata}

This modified cellular automata technique is a means of breaking up large, difficult to solve, multi-physics models into smaller, more easily digested bits. It requires feeding the result of each single/reduced multi-physics system into the next, and solving iteratively through small, appropriately chosen time steps for the physics involved. A single advancement is represented pictorially in Figure 2.4. This rule based method of advancing each of the individual physics independently has the advantage of enabling each of the physics to be solved using the technique best suited to it. The mathematics of this method proceeds in a stepwise fashion and therefore lacks simultaneity, however. Thus, the time steps must be small to avoid the risk of nonphysical results. 


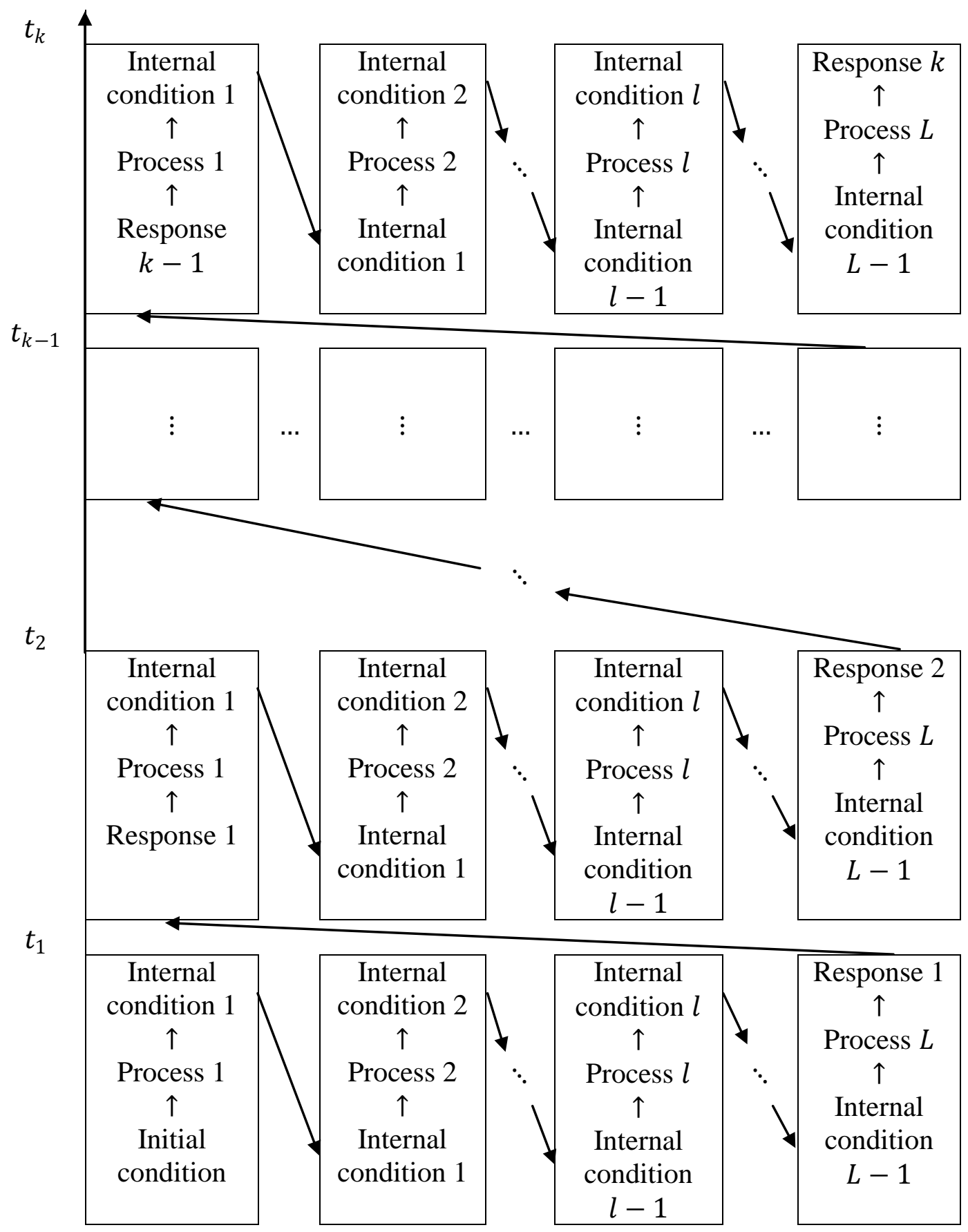

Figure 2.4: Pictorial representation of the modified cellular automata method.

\subsection{Parameter Estimation}

There are two major types of inverse problems. Both of the inverse problems are mathematically ill-posed. The solution is not necessarily unique, and very frequently is not 
stable for small changes in the input conditions. The two types of inverse problem are the estimation of the forcing functions, and the estimation of system parameters. Both require the estimation of something required to cause the observed response in the data. In the estimation of the forcing function case, the system is fully characterized. By knowing the response of the system, one can estimate what the excitation must have been. This problem is graphically represented in Figure 2.5.

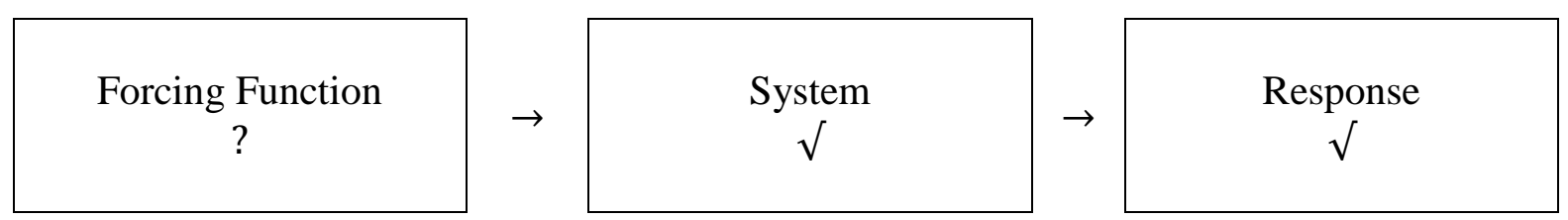

Figure 2.5: Schematic representation of the estimation of the forcing function.

The other type of inverse problem is system parameter estimation. Parameter estimation problems are the class of problem where the excitation is known and the response is measured. By knowing the response of the system to the excitation, one can estimate the system parameters that caused the response. This is the case that is discussed in this thesis. The distribution of contacts and the total real area of contact are physical parameters of the system. Parameter estimation frequently exhibits instability in its mathematics because of singular or near-singular matrices that appear in the math. The means by which these are handled are discussed in chapter 5. A schematic representation of the parameter estimation problem is shown in Figure 2.6.

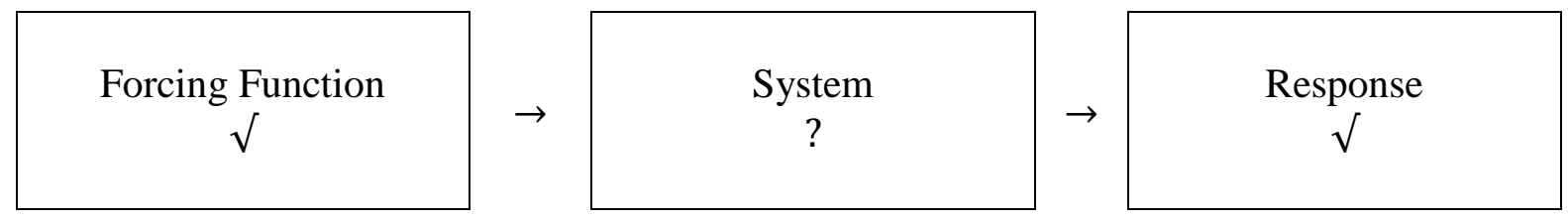

Figure 2.6: Schematic representation of the estimation of system parameters. 


\section{Physical Formulation of the Direct Model}

The first step in determining the parameter values of an incompletely defined system is to define a functional mathematical model of the physical system. This chapter develops the models that will be used in the analysis of the two dimensional, two body $(2 \mathrm{D}, 2 \mathrm{~B})$ frictional system and its degenerates.

\subsection{D, 2B System}

The most general system that will be considered is the $2 \mathrm{D}, 2 \mathrm{~B}$ problem. This allows easier depictions of the system, as well as reduced computational time.

\subsubsection{General Physical Schematic}

Figure 3.1 depicts a schematic view of the unwrapped physical system of Figure 2.2. The top portion of the figure depicts the geometric arrangement with the dimensions and coordinate system shown. The double wavy lines at the left and right of the second body indicate that the boundary wraps around to the other side. This notation is used throughout the figures of this paper.

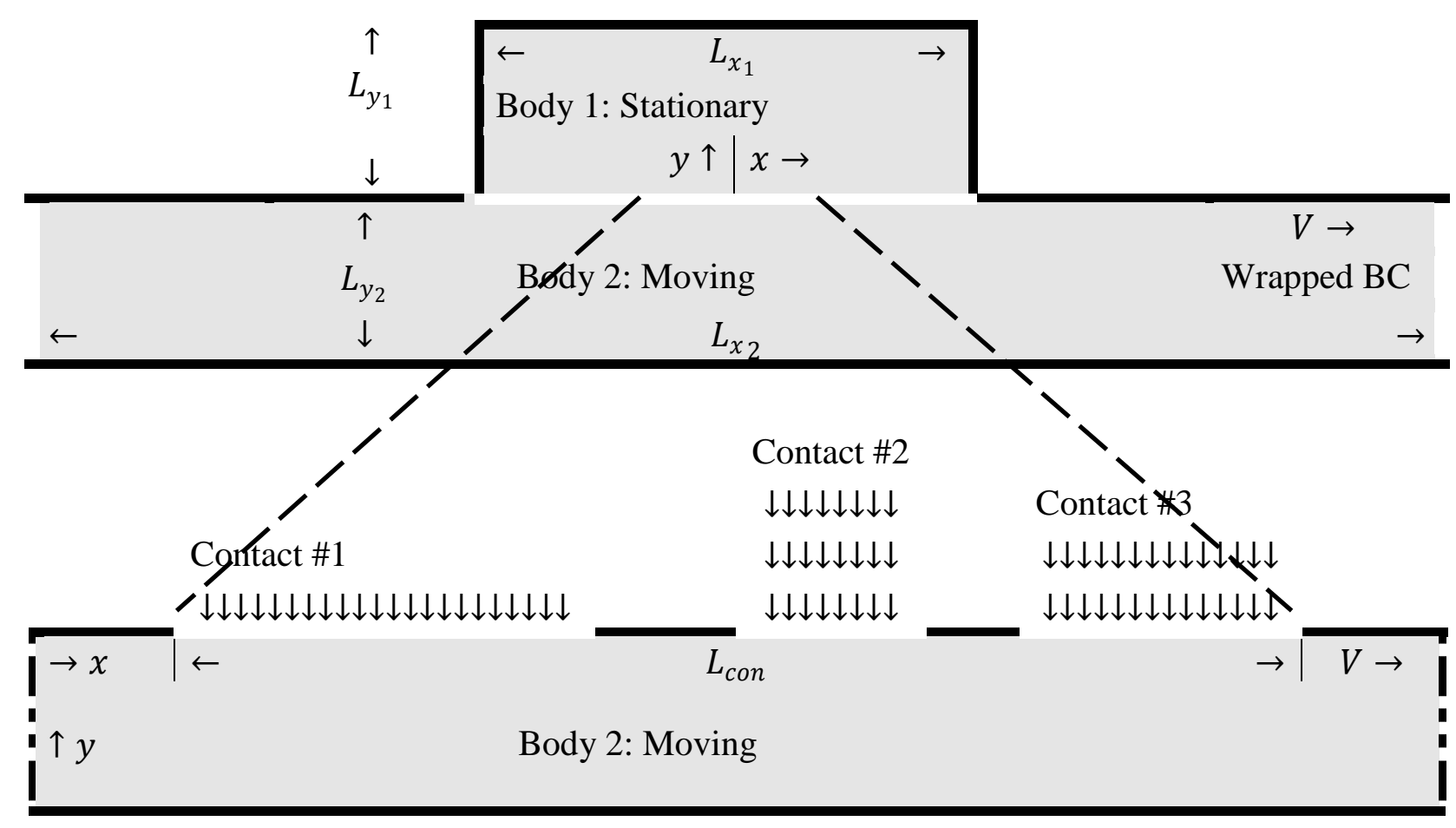

Figure 3.1: Schematic portrayal of the physical system in Figure 2.2.

Three contacts of varying intensity are depicted. 
The bottom portion of the figure depicts a close up of the nominal contact region of the second body. One of the objectives of the research is to determine approximately what the real contact area must be to produce the observed temperatures. The figure depicts individual contacts of varying intensity and size. The real contact area is the sum of each of the individual contact areas.

\subsubsection{General Contact Area Profile}

The contacts can be thermally represented as a heat flux distribution (e.g. as uniform distributions for plastic contacts, or Hertzian distributions for elastic contacts). The only region where this distribution can be positive is over the nominal contact zone. Points of zero contact between the two bodies are, by definition, zero. Thus, the mathematical representation is

$$
q_{\text {fric }}^{\prime \prime}(x, t)=q_{\text {fric }} \cdot f_{d}(x, t)
$$

The total power dissipated by friction $\left(q_{\text {fric }}\right)$ is calculated by:

$$
q_{\text {fric }}=\mu_{k} F_{n} V
$$

where $\mu_{k}$ is the sliding coefficient of friction, $F_{n}$ is the normal force, and $V$ is the velocity of the solid. The contact distribution, $f_{d}(x, t)$, behaves according to the following two constraints:

$$
\begin{gathered}
L_{z} \int_{L_{c o n}} f_{d}(x, t) d x=1 \\
f_{d}(x, t)=0, \quad x \text { outside contact zone }
\end{gathered}
$$

This contact distribution has units of $\frac{1}{\text { lengt } h^{2}}$. This distribution will give insight to multiple aspects of the contact region. It depicts the shape of the actual heat flux distribution. This distribution will also have a shape similar to that of the pressure distribution, as mechanical work is the means by which the heat is evolved. Because the power dissipated as heat is modeled as equation 3.2, the pressure and heat flux profiles will necessarily be proportional to each other. Sample shapes are provided below in Figure 3.2. Each of the distributions integrates to unity. 

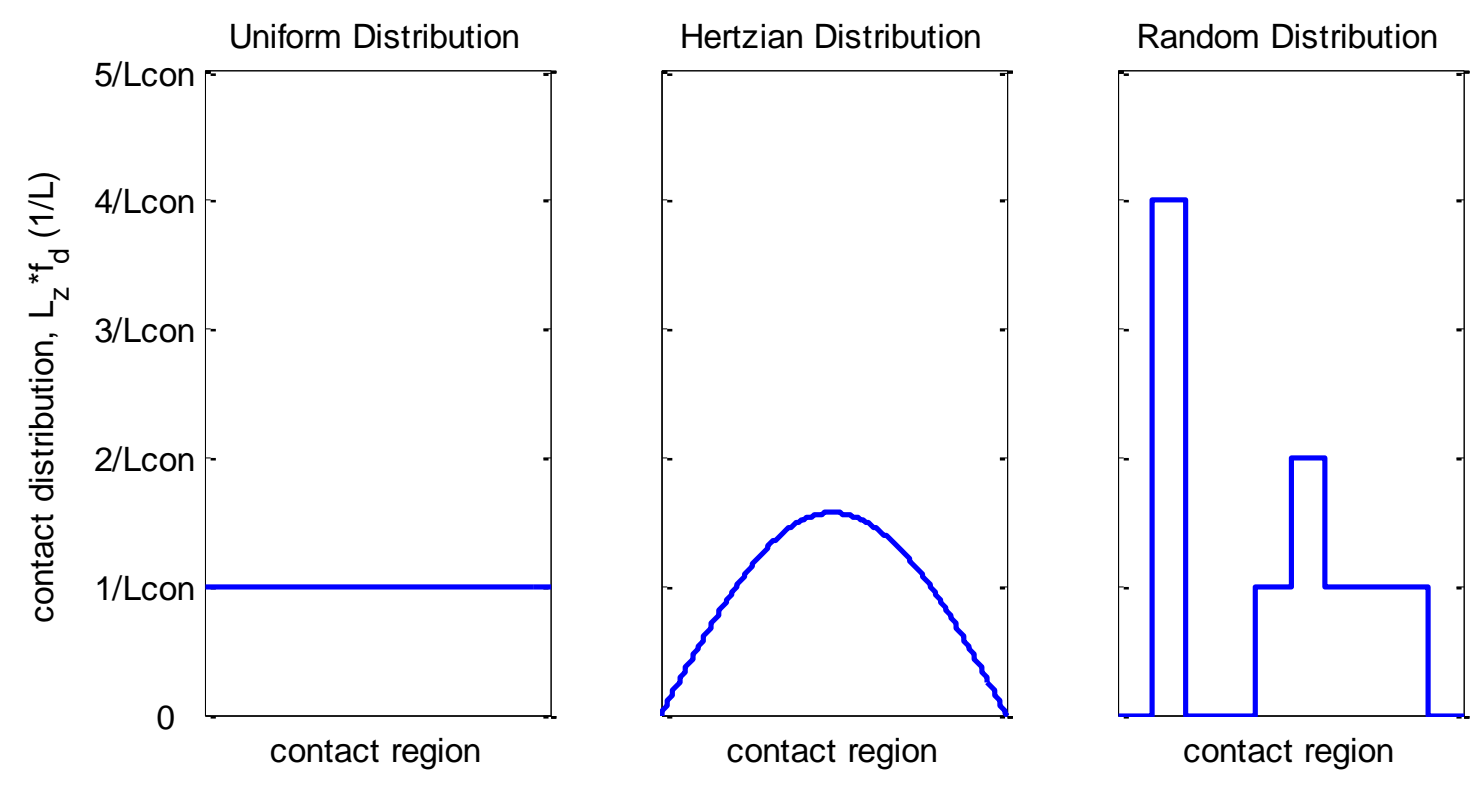

Figure 3.2: Sample contact distributions.

The distributions show how bodies can come into contact over the nominal contact region. The 'multiple contacts' distribution is the viewpoint paradigm with which this paper approaches the problem. This paradigm allows for multiple, irregularly shaped contacts, with the minimum resolution being the size of the spatial discretization of the model.

Further, since the contact distribution was derived on the basis that it is zero in regions of zero contact, it also gives insight into the size of the real contact area. One can look at the distribution as being such that the regions with a large relative value of the contact distribution are contacts. The converse is also true, where low value regions of the contact distribution are where the two bodies are not in contact.

\subsubsection{Formulation of the 2D, 2B Problem}

Now that the heat addition through friction is represented as a heat flux distribution, the system can now be modeled as a standard heat transport process. The depiction of the problem setup can be found in Figure 3.3. Figure 3.3 depicts the flows of energy in and out of the control volumes in each body. One can see that the stationary Body 1 undergoes a purely conductive behavior, while the moving Body 2 exhibits advective behavior in addition to conduction. 


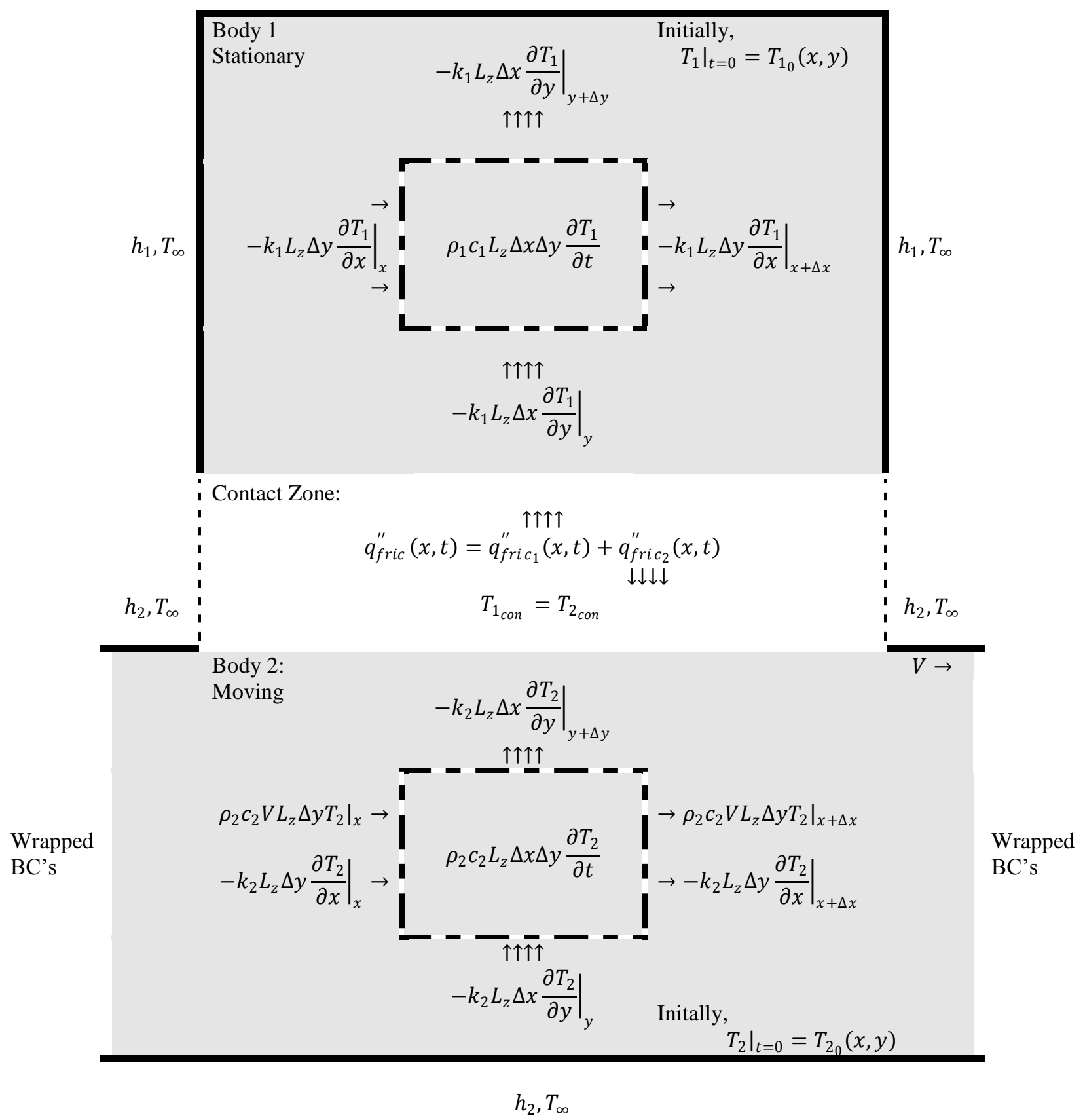

Figure 3.3: Geometric layout of the heat transfer equations by control volume analysis.

Visible in this figure are all of the transport flows in and out of general internal differential control volumes (of dimensions $\left.\Delta x, \Delta y, L_{z}\right)$. Further, boundary and initial conditions are placed as appropriate.

Of further note from this image is the description of the boundary conditions. The boundary conditions are shown near the surface where they take effect. Convective boundary conditions are denoted simply with the coefficient and the temperature. 
Of major importance are the coupling conditions at the contacts, namely the continuity of temperature and conservation of energy. Conservation of energy requires that the total frictional heat must be divided between the two bodies. The frictional heat flux, $q_{\text {fric }}^{\prime \prime}$, is considered as known, and was characterized in section 3.1.2. However, the heat partition, $q_{\text {fric } 1}^{\prime \prime}$ and $q_{\text {fric }}^{\prime \prime}$, are unknown and must be determined as part of the overall solution.

The actual governing equations for the two bodies in Figure 3.3 can be readily deduced at this point. By summing the flows in and out of the differential control volume as shown in Figure 3.3 in body 1, one gains the following relationship:

$$
\begin{aligned}
\rho_{1} c_{1} L_{z} \Delta x & \Delta y \frac{\partial T_{1}}{\partial t} \\
= & -k_{1} L_{z}\left(\Delta y\left(\left.\frac{\partial T_{1}}{\partial x}\right|_{x}-\left(\left.\frac{\partial T_{1}}{\partial x}\right|_{x}+\left.\frac{\partial^{2} T_{1}}{\partial x^{2}}\right|_{x} \Delta x\right)\right)\right. \\
& \left.+\Delta x\left(\left.\frac{\partial T_{1}}{\partial y}\right|_{y}-\left(\left.\frac{\partial T_{1}}{\partial y}\right|_{y}+\left.\frac{\partial^{2} T_{1}}{\partial y^{2}}\right|_{y} \Delta y\right)\right)\right)
\end{aligned}
$$

By a few algebra steps, one can reduce this to acquire Equation 3.6:

$$
\frac{\partial T_{1}}{\partial t}=\alpha_{1}\left(\frac{\partial^{2} T_{1}}{\partial x^{2}}+\frac{\partial^{2} T_{1}}{\partial y^{2}}\right)
$$

This is the anticipated result of a two dimensional, purely conductive system. This equation governs the flow of heat inside body 1.

Body 2 can be handled much the same as body 1, with the exception that it has an additional pair of energy flows as a result of advection through the sides of the control volume. The following relationship is the result of summing the heat flows in and out of the control volume in body 2 in Figure 3.3. 


$$
\begin{aligned}
\rho_{2} c_{2} L_{z} \Delta x \Delta y \frac{\partial T_{2}}{\partial t} \\
\quad=-k_{2} L_{z}\left(\Delta y\left(\left.\frac{\partial T_{2}}{\partial x}\right|_{x}-\left(\left.\frac{\partial T_{2}}{\partial x}\right|_{x}+\left.\frac{\partial^{2} T_{2}}{\partial x^{2}}\right|_{x} \Delta x\right)\right)\right. \\
\left.+\Delta x\left(\left.\frac{\partial T_{2}}{\partial y}\right|_{y}-\left(\left.\frac{\partial T_{2}}{\partial y}\right|_{y}+\left.\frac{\partial^{2} T_{2}}{\partial y^{2}}\right|_{y} \Delta y\right)\right)\right) \\
+\rho_{2} c_{2} V L_{z} \Delta y\left(\left.T_{2}\right|_{x}-\left(\left.T_{2}\right|_{x}+\left.\frac{\partial T_{2}}{\partial x}\right|_{x} \Delta x\right)\right)
\end{aligned}
$$

By a few algebra steps, one can reduce this to acquire Equation 3.8:

$$
\frac{\partial T_{2}}{\partial t}=\alpha_{2}\left(\frac{\partial^{2} T_{2}}{\partial x^{2}}+\frac{\partial^{2} T_{2}}{\partial y^{2}}\right)-V \frac{\partial T_{2}}{\partial x}
$$

This equation is the anticipated result of a two dimensional, conductive-advective system. This equation governs the flow of heat inside body 2 .

\subsubsection{D, 2B Formulation Results}

The results of the formulation can be represented in a form where the governing equations and boundary/initial conditions are located in the representation where they physically perform their action. For instance, the left and right hand (x) boundary conditions for body 1 appear in Figure 3.4 next to the edges of the solid where they would take effect. This representation should readily demonstrate the transformation of Figure 3.3 from setting up the control volume analysis to a final representation of the formulation of the problem. 


$$
\begin{aligned}
& -k_{1} \frac{\partial T_{1}}{\partial y}=h_{1}\left(T_{1}-T_{\infty}\right) \\
& \text { @y }=L_{y_{1}} \\
& \text { Body 1: Stationary } \\
& k_{1} \frac{\partial T_{1}}{\partial x}=h_{1}\left(T_{1}-T_{\infty}\right) \\
& @ x=-\frac{L_{c o n}}{2} \\
& \frac{\partial T_{1}}{\partial t}=\alpha_{1}\left(\frac{\partial^{2} T_{1}}{\partial x^{2}}+\frac{\partial^{2} T_{1}}{\partial y^{2}}\right) \\
& \left.T_{1}\right|_{t=0}=T_{1_{0}}(x, y) \\
& -k_{1} \frac{\partial T_{1}}{\partial x}=h_{1}\left(T_{1}-T_{\infty}\right) \\
& @ x=\frac{L_{c o n}}{2} \\
& \left\{\begin{array}{c}
q_{\text {fric }}^{\prime \prime}(x, t)=-\left.k_{1} \frac{\partial T_{1}}{\partial y}\right|_{y=0}+\left.k_{2} \frac{\partial T_{2}}{\partial y}\right|_{y=0} \\
T_{1_{c o n}}=T_{2 c o n} \\
\hdashline \leftarrow \downarrow \downarrow \downarrow \\
\hdashline------L_{c o n}-------\rightarrow
\end{array}\right. \\
& -k_{2} \frac{\partial T_{2}}{\partial y}=h_{2}\left(T_{2}-T_{\infty}\right) \\
& @ y=0, x<-\frac{L_{c o n}}{2} \\
& \frac{\partial T_{2}}{\partial t}=\alpha_{2}\left(\frac{\partial^{2} T_{2}}{\partial x^{2}}+\frac{\partial^{2} T_{2}}{\partial y^{2}}\right)-V \frac{\partial T_{2}}{\partial x} \\
& \left.T_{2}\right|_{t=0}=T_{2_{0}}(x, y) \\
& k_{2} \frac{\partial T_{2}}{\partial x}=h_{2}\left(T_{2}-T_{\infty}\right) \\
& @ y=-L_{y_{2}}
\end{aligned}
$$

Figure 3.4: 2D, 2B formulation summarized by physical location.

The key items to note are the additional advective heat transfer mechanism in body 2 and the partition of heat between the two bodies.

Table 3.1 shows the formulation of the problem expressed in a more conventional form, where one can see the complete set of equations and boundary/initial conditions for the transport equations involving the two bodies. It is demonstrated that each body has 2 boundary conditions in each direction and an initial temperature distribution, as required by the different derivatives involved. 
Table 3.1: 2D, 2B formulation summarized in tabular form.

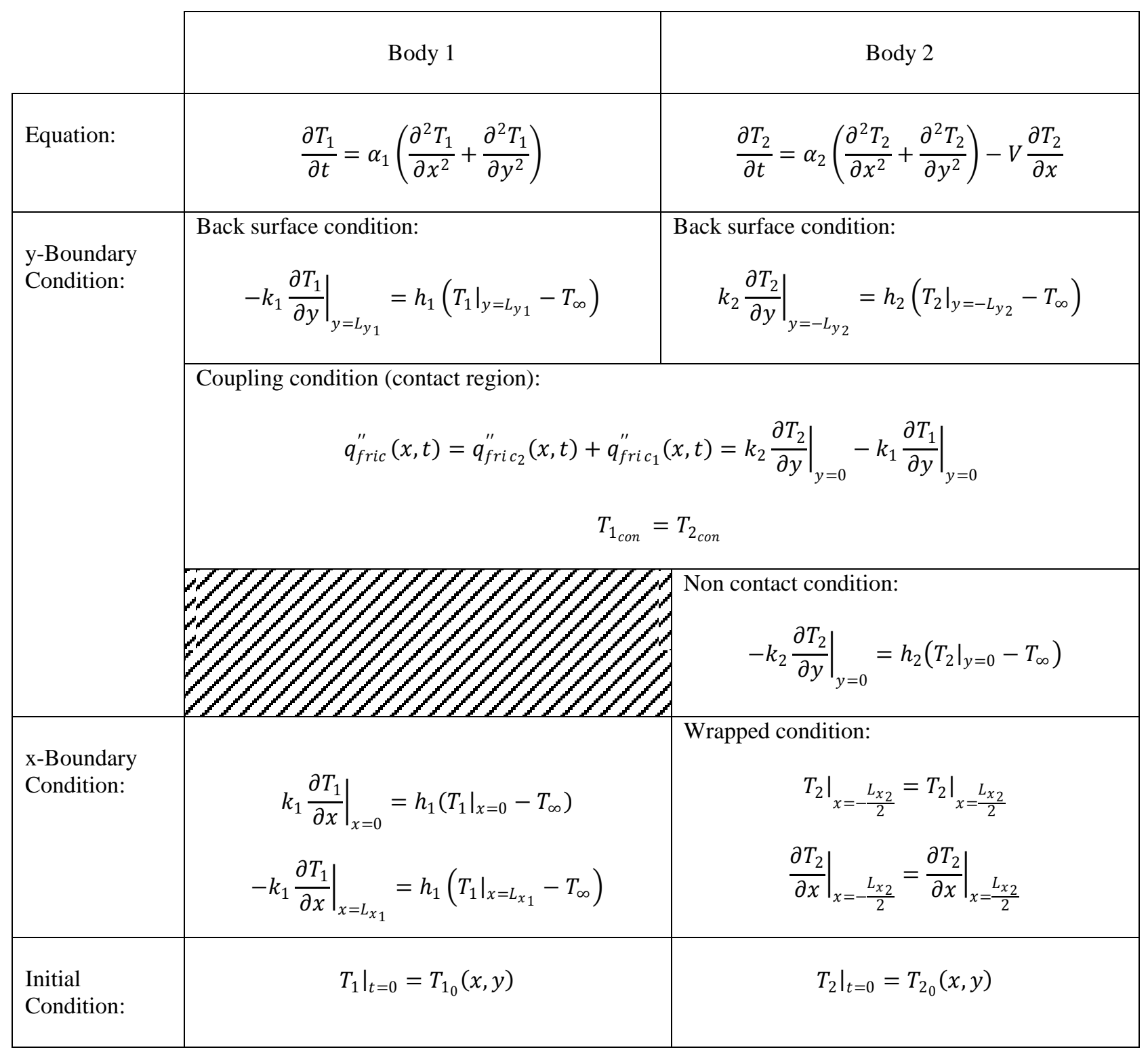

\subsection{Formulation of the 2D, 1B Problem}

In order to arrive at the two dimensional, one body (2D, 1B) formulation, one simply must degenerate the $2 \mathrm{D}, 2 \mathrm{~B}$ formulation results. In this case, in order to remove the second (non-moving) body, one needs to make the simplifying assumption that all of the heat evolved is transported into the moving body. The results in Figure 3.5, therefore, look very similar to the results achieved in Figure 3.4. The primary differences are that, obviously, the stationary body is 
removed, and that the coupling conditions have been reduced to a heat flux (as opposed to the coupled conditions).

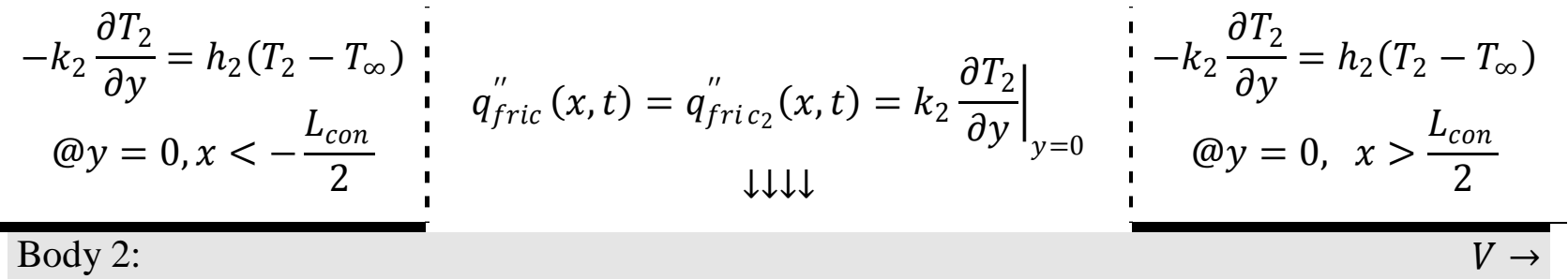

$$
\begin{aligned}
& \text { Moving } \frac{\partial T_{2}}{\partial t}=\alpha_{2}\left(\frac{\partial^{2} T_{2}}{\partial x^{2}}+\frac{\partial^{2} T_{2}}{\partial y^{2}}\right)-V \frac{\partial T_{2}}{\partial x} \\
& \left.T_{2}\right|_{t=0}=T_{2_{0}}(x, y) \\
& k_{2} \frac{\partial T_{2}}{\partial x}=h_{2}\left(T_{2}-T_{\infty}\right) \\
& @ y=-L_{y_{2}}
\end{aligned}
$$

Figure 3.5: 2D, 1B formulation summarized by physical location.

Following in the form of Table 3.1, Table 3.2 shows the formulation expressed in the same tabularized form. Once again, all boundary and initial conditions are accounted for as required by the formulated equation for the moving body. 
Table 3.2: 2D, 1B formulation summarized in tabular form.

\begin{tabular}{|c|c|}
\hline & Body 2 \\
\hline Equation: & $\frac{\partial T_{2}}{\partial t}=\alpha_{2}\left(\frac{\partial^{2} T_{2}}{\partial x^{2}}+\frac{\partial^{2} T_{2}}{\partial y^{2}}\right)-V \frac{\partial T_{2}}{\partial x}$ \\
\hline \multirow[t]{3}{*}{$\begin{array}{l}\text { y-Boundary } \\
\text { Condition: }\end{array}$} & $\begin{array}{l}\text { Back surface condition: } \\
\qquad\left.k_{2} \frac{\partial T_{2}}{\partial y}\right|_{y=-L_{y_{2}}}=h_{2}\left(\left.T_{2}\right|_{y=-L_{y_{2}}}-T_{\infty}\right)\end{array}$ \\
\hline & $\begin{array}{l}\text { Contact condition: } \\
\qquad q_{\text {fric }}^{\prime \prime}(x, t)=q_{f r i c_{2}}^{\prime \prime}(x, t)=\left.k_{2} \frac{\partial T_{2}}{\partial y}\right|_{y=0}\end{array}$ \\
\hline & $\begin{array}{l}\text { Non contact condition: } \\
\qquad-\left.k_{2} \frac{\partial T_{2}}{\partial y}\right|_{y=0}=h_{2}\left(\left.T_{2}\right|_{y=0}-T_{\infty}\right)\end{array}$ \\
\hline \multirow[t]{2}{*}{$\begin{array}{l}\text { x-Boundary } \\
\text { Condition: }\end{array}$} & Wrapped condition: \\
\hline & $\left.\frac{\partial T_{2}}{\partial x}\right|_{x=-\frac{L_{x_{2}}}{2}}=\left.\frac{\partial T_{2}}{\partial x}\right|_{x=\frac{L_{x_{2}}}{2}}$ \\
\hline $\begin{array}{l}\text { Initial } \\
\text { Condition: }\end{array}$ & $\left.T_{2}\right|_{t=0}=T_{2_{0}}(x, y)$ \\
\hline
\end{tabular}

\subsection{Formulation of the 1D, 2B Problem}

If the solids involved are small enough in the ' $y$ ' direction, such that the gradients are negligible, then the model can be considered one dimensional. This statement implies that:

$$
T(x, y, t) \cong T(x, t)
$$

In order to arrive at the one dimensional, two body (1D, 2B) formulation results, one must degenerate the 2D, 2B formulation results. For this particular case, it is mathematically more difficult to attain. Conceptually, one simply integrates over the y-domain for each equation. The major steps of these integrations are shown. 
For body 1 , the integration is over the domain $\left[0, L_{y_{1}}\right]$, thus the integral setup is as follows:

$$
\int_{y=0}^{L_{y_{1}}} \rho_{1} c_{1} \Delta x L_{z} \frac{\partial T_{1}}{\partial t} d y=\int_{y=0}^{L_{y_{1}}} k_{1} \Delta x L_{z}\left(\frac{\partial^{2} T_{1}}{\partial x^{2}}+\frac{\partial^{2} T_{1}}{\partial y^{2}}\right) d y
$$

By performing the integration and assuming negligible temperature changes in the ' $y$ ' direction, the result is:

$$
\rho_{1} c_{1} \Delta x L_{z} \frac{\partial T_{1}}{\partial t} L_{y_{1}}=k_{1} \Delta x L_{z} \frac{\partial^{2} T_{1}}{\partial x^{2}} L_{y_{1}}+k_{1} \Delta x L_{z}\left(\left.\frac{\partial T_{1}}{\partial y}\right|_{y=L_{y_{1}}}-\left.\frac{\partial T_{1}}{\partial y}\right|_{y=0}\right)
$$

By substituting in the boundary conditions and re-arranging algebraically, the final result is:

$$
\frac{\partial T_{1}}{\partial t}=\alpha_{1} \frac{\partial^{2} T_{1}}{\partial x^{2}}-\frac{h_{1}}{\rho_{1} c_{1} L_{y_{1}}}\left(T_{1}-T_{\infty}\right)+\frac{q_{\text {fric }, 1}^{\prime \prime}(x, t)}{\rho_{1} c_{1} L_{y_{1}}}
$$

For body 2 , the integration is over the domain $\left[-L_{y_{2}}, 0\right]$. The math is, again, much the same, however the $y=0$ boundary condition is more complex. The integral setup is as follows:

$$
\begin{aligned}
\int_{y=-L_{y_{2}}}^{0} & \rho_{2} c_{2} \Delta x L_{z} \frac{\partial T_{2}}{\partial t} d y \\
& =\int_{y=-L_{y_{2}}}^{0} k_{2} \Delta x L_{z}\left(\frac{\partial^{2} T_{2}}{\partial x^{2}}+\frac{\partial^{2} T_{2}}{\partial y^{2}}\right) d y \\
& -\int_{y=-L_{y_{2}}}^{0} V \rho_{2} c_{2} \Delta x L_{z} \frac{\partial T_{2}}{\partial x} d y
\end{aligned}
$$

By integrating and neglecting ' $y$ ' direction variations, the result is:

$$
\begin{aligned}
\rho_{2} c_{2} \Delta x L_{z} & L_{y_{2}} \frac{\partial T_{2}}{\partial t} \\
& =k_{2} \Delta x L_{z} L_{y_{2}} \frac{\partial^{2} T_{2}}{\partial x^{2}}+k_{2} \Delta x L_{z}\left(\left.\frac{\partial T_{2}}{\partial y}\right|_{y=0}-\left.\frac{\partial T_{2}}{\partial y}\right|_{y=-L_{y_{2}}}\right) \\
& -V \rho_{2} c_{2} \Delta x L_{z} L_{y_{2}} \frac{\partial T_{2}}{\partial x}
\end{aligned}
$$

By substituting in the boundary conditions and re-arranging algebraically, the final result is:

$$
\begin{aligned}
\frac{\partial T_{2}}{\partial t}= & \alpha 2 \frac{\partial^{2} T_{2}}{\partial x^{2}}+\frac{q_{f r i c, 2}^{\prime \prime}(x, t)}{\rho_{2} c_{2} L_{y 2}}-V \frac{\partial T_{2}}{\partial x} \\
& -\left(H\left(-\frac{L_{c o n}}{2}-x\right)+H\left(x-\frac{L_{c o n}}{2}\right)+1\right) \frac{h_{2}}{\rho_{2} c_{2} L_{y_{2}}}\left(T_{2}-T_{\infty}\right)
\end{aligned}
$$


Again, one can use the same depiction method in Figure 3.4 for the degenerated case in Figure 3.6. The integrations performed above remove the ' $y$ ' functionality of the equations and leave the system as a pair of coupled 1D systems. As compared to Figure 3.4, Figure 3.6 readily demonstrates how the ' $y$ ' boundary conditions are absorbed into the equations over the integration, thus leaving only the initial conditions and the ' $x$ ' boundary conditions. As an additional mathematical note, the multiplier on the convective loss term exists to state when convective loss is acting on both surfaces or not.

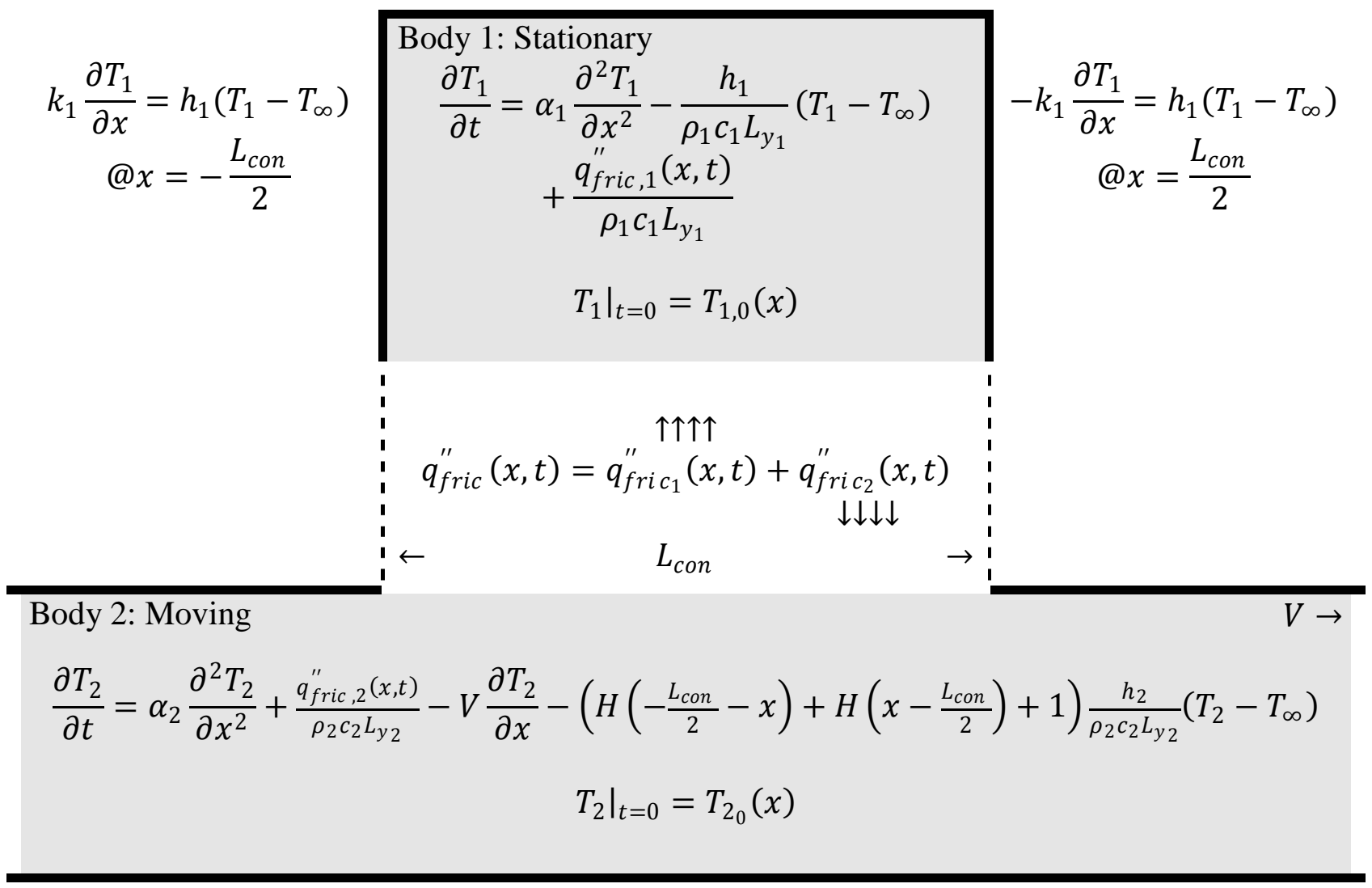

Figure 3.6: 1D, 2B formulation summarized by physical location.

The above formulation is again tabularized in Table 3.3. It again demonstrates the reduction of the equations to a transient, 1D form. Only present are the ' $x$ ' boundary conditions and the initial conditions, because in the process of integration across the ' $y$ ' direction, those boundary conditions were used. 
Table 3.3: 1D, 2B formulation summarized in tabular form.

\begin{tabular}{|c|c|c|}
\hline & Body 1 & Body 2 \\
\hline Equation: & $\frac{\partial T_{1}}{\partial t}=\alpha_{1} \frac{\partial^{2} T_{1}}{\partial x^{2}}-\frac{h_{1}}{\rho_{1} c_{1} L_{y_{1}}}\left(T_{1}-T_{\infty}\right)+\frac{q_{f r i c_{1}}^{\prime \prime}(x, t)}{\rho_{1} c_{1} L_{y 1}}$ & $\begin{aligned} \frac{\partial T_{2}}{\partial t}=\alpha_{2} & \frac{\partial^{2} T_{2}}{\partial x^{2}}+\frac{q_{f r i c_{2}}^{\prime \prime}(x, t)}{\rho_{2} c_{2} L_{y 2}}-V \frac{\partial T_{2}}{\partial x} \\
& -\frac{h_{2}}{\rho_{2} c_{2} L_{y 2}}\left(T_{2}-T_{\infty}\right) \\
& \cdot\left(H\left(-\frac{L_{c o n}}{2}-x\right)+H\left(x-\frac{L_{c o n}}{2}\right)+1\right)\end{aligned}$ \\
\hline $\begin{array}{l}\text { x-Boundary } \\
\text { Condition: }\end{array}$ & $\begin{array}{c}\left.k_{1} \frac{\partial T_{1}}{\partial x}\right|_{x=0}=h_{1}\left(\left.T_{1}\right|_{x=0}-T_{\infty}\right) \\
-\left.k_{1} \frac{\partial T_{1}}{\partial x}\right|_{x=L_{x_{1}}}=h_{1}\left(\left.T_{1}\right|_{x=L_{x_{1}}}-T_{\infty}\right)\end{array}$ & $\begin{array}{l}\text { Wrapped Condition: } \\
\qquad\left.T_{2}\right|_{x=-\frac{L_{x_{2}}}{2}}=\left.T_{2}\right|_{x=\frac{L_{x_{2}}}{2}} \\
\qquad\left.\frac{\partial T_{2}}{\partial x}\right|_{x=-\frac{L_{x_{2}}}{2}}=\left.\frac{\partial T_{2}}{\partial x}\right|_{x=\frac{L_{x_{2}}}{2}}\end{array}$ \\
\hline $\begin{array}{l}\text { Initial } \\
\text { Condition: }\end{array}$ & $\left.T_{1}\right|_{t=0}=T_{1_{0}}(x)$ & $\left.T_{2}\right|_{t=0}=T_{2_{0}}(x)$ \\
\hline
\end{tabular}

\subsection{Formulation of the 1D, 1B Problem}

In order to arrive at the one dimensional, one body (1D, 1B) formulation, the static body must be removed from the $1 \mathrm{D}, 2 \mathrm{~B}$ formulation. The reduction is much the same procedurally as the reduction to the 2D, 1B formulation from the 2D, 2B formulation. Figure 3.7 shows this formulation geometrically. As with the previous 1B case, all of the heat evolved at the interface enters the moving body.

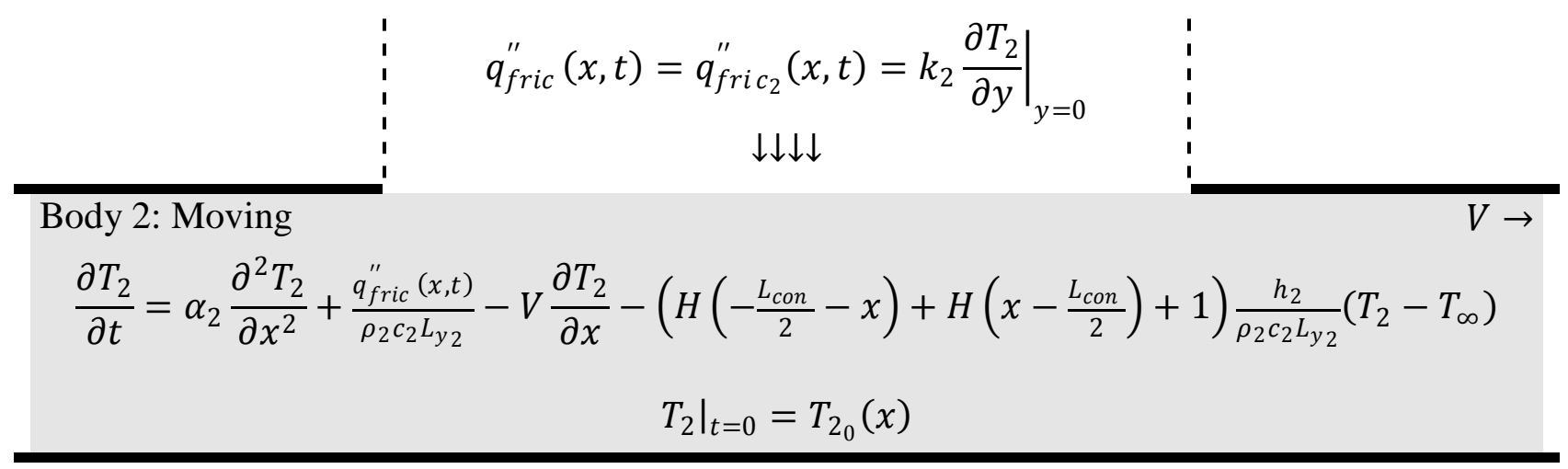

Figure 3.7: 1D, 1B formulation summarized by physical location. 
Table 3.4 shows the formulation in a tabular form. One can readily see that all required boundary and initial conditions are present.

Table 3.4: 1D, 1B formulation summarized in tabular form.

\begin{tabular}{|c|c|}
\hline & Body 2 \\
\hline Equation: & $\begin{aligned} \frac{\partial T_{2}}{\partial t}= & \alpha_{2} \frac{\partial^{2} T_{2}}{\partial x^{2}}+\frac{q_{f r i c}^{\prime \prime}(x, t)}{\rho_{2} c_{2} L_{y 2}}-V \frac{\partial T_{2}}{\partial x} \\
& -\left(H\left(-\frac{L_{c o n}}{2}-x\right)+H\left(x-\frac{L_{c o n}}{2}\right)+1\right) \frac{h_{2}}{\rho_{2} c_{2} L_{y_{2}}}\left(T_{2}-T_{\infty}\right)\end{aligned}$ \\
\hline \multirow[t]{2}{*}{$\begin{array}{l}\text { x-Boundary } \\
\text { Condition: }\end{array}$} & Wrapped condition: \\
\hline & $\left.\frac{\partial T_{2}}{\partial x}\right|_{x=-\frac{L_{x_{2}}}{2}}=\left.\frac{\partial T_{2}}{\partial x}\right|_{x=\frac{L_{x_{2}}}{2}}$ \\
\hline Initial Condition: & $\left.T_{2}\right|_{t=0}=T_{2_{0}}(x)$ \\
\hline
\end{tabular}




\section{Solution of the Direct Problem via Modified Cellular Automata}

This chapter deals with the development of the numerical solution to the direct problems formulated in Chapter 3. It will cover the modified cellular automata method and the rules developed for this particular set of physics.

\subsection{Basic Concept}

The method of cellular automata is a method of breaking physics up into discrete, rule based, solutions that are applied to a given set of conditions in a serial manner. The formal method of cellular automata requires the entire system to be discrete, including the state variable. Temperature, by its nature, is not a naturally discrete state variable, and thus to use cellular automata, the state variable would have to be artificially discretized. This behavior is undesirable. However, the concept of breaking a difficult equation up (similar to operator splitting) into easier pieces and solving them in a serial manner is very attractive when computational times for the full system start to accumulate rapidly. Thus, we use a modified cellular automata method to arrive at the solution to the direct problem.

The method of modified cellular automata is a technique of breaking up large, difficult to solve, multi-physics models into smaller, more easily digested mathematical pieces. This method requires feeding the result of each single (or reduced multi-) physics rule into the next, and solving the set of rules iteratively through small, appropriately chosen time steps. As a result of the breaking up of the physics into smaller rules, solved in a serial, rather than simultaneous, method, the order of the rule employment can have a small impact on the end result. However, it has been shown [39] that as the time step over which each rule is solved consecutively gets smaller, the differences in the results are reduced. Further, it was shown for the given examples that the order did not affect the results enough to make them physically unrealistic.

The modified cellular automata method allows the user to draw from many well developed and understood solution methods. Finite difference methods, analytical solutions, and Runge-Kutta solvers are all available for use in this overall technique. After developing the mathematical model, one must then determine the breakdown of the mathematics into its simpler rules for the employment of this method. As an initial step, one must discretize the independent variable dimensions (space and time). Time is already discretized as a result of the cellular approach where the multi-physics are simulated for each major time step. Space also needs to be 
discretized based on any requirements regarding the spatial resolution. This discrete grid can visually be represented in Figure 4.1 .

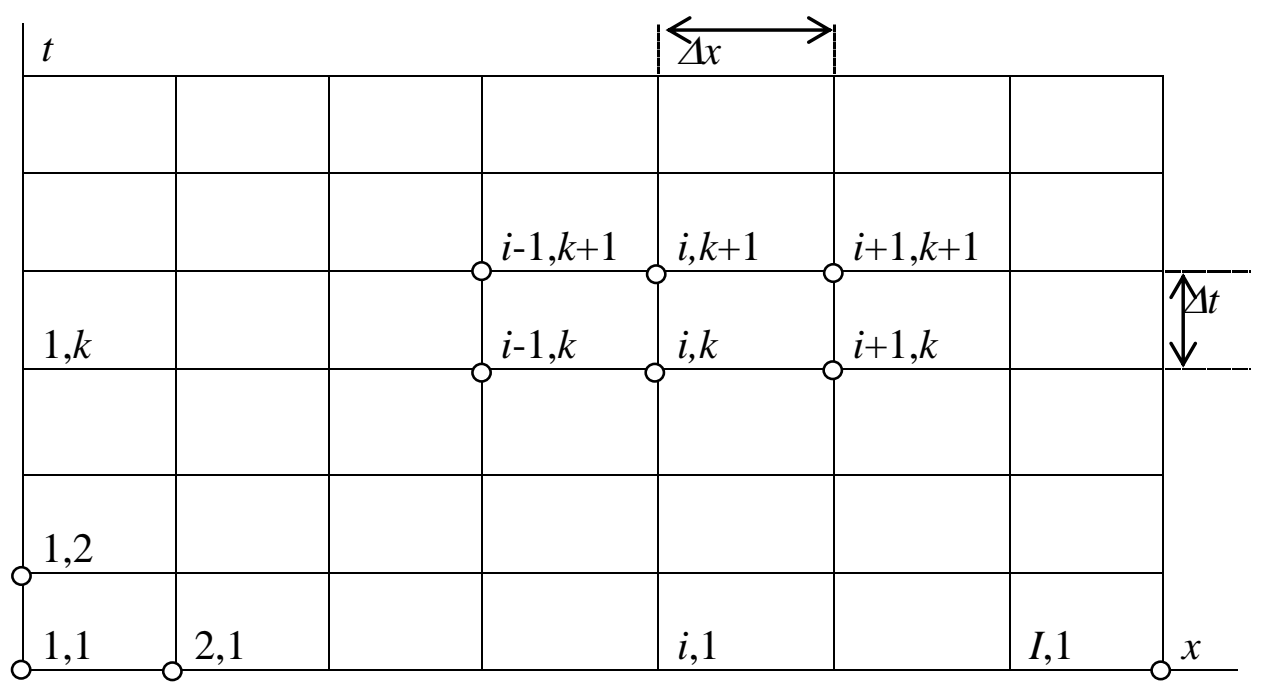

Figure 4.1: Time and space cellular grid example.

Time is indexed by the ' $k$ ' variable and space is indexed by ' $i$ ' (with ' $I$ ' indicating final spatial index).

More rigorously, the proposed method is generally done as follows. First, the general fundamental governing equation for the system is:

$$
\frac{\partial T}{\partial t}=\sum_{l=1}^{L} \boldsymbol{f}_{l}(T)
$$

where $T$ is the state variable (in this case, the temperature distribution of the medium), and $\boldsymbol{f}_{l}(\quad)$ is the $l^{\text {th }}$ operator (process) of the multi-physical system. In the cases looked at in this paper, it could be advection, diffusion, or source effects. Thus, the $l^{\text {th }}$ fundamental process of the system is:

$$
\frac{\partial T}{\partial t}=\boldsymbol{f}_{l}(T), \quad l=1,2 \ldots L
$$

For each of these processes, one must develop a solution technique. Many will be analytically solvent when broken down appropriately. Those that are not should be reasonably easy to solve using other, well understood, methods such as Runge-Kutta or finite differencing. The solution of the individual process can be represented:

$$
T_{k+1}=\mathcal{F}_{l}\left(T_{k}\right)
$$


where $\mathcal{F}$ is the actual rule that advances the solution one major time step, $T_{k+1}$ is the advanced solution, and $T_{k}$ is the previous iteration's result. As previously stated, each of the individual $\mathcal{F}_{l}$ 's can utilize any number of solution techniques, ranging from purely analytical solutions to Runge-Kutta methods. For any multi-physics system, while solving for a single time step, the result of each rule is fed into the next until each rule is used. Thus, for multiple physics, the solution for a single step is:

$$
\begin{gathered}
T_{k, 1}=\mathcal{F}_{1}\left(T_{k}\right) \\
T_{k, 2}=\mathcal{F}_{2}\left(T_{k, 1}\right) \\
T_{k, 3}=\mathcal{F}_{3}\left(T_{k, 2}\right) \\
\vdots \\
T_{k, l}=\mathcal{F}_{l}\left(T_{k, l-1}\right) \\
\vdots \\
T_{k, L-1}=\mathcal{F}_{L-1}\left(T_{k, L-2}\right) \\
T_{k+1}=\mathcal{F}_{L}\left(T_{k, L-1}\right)
\end{gathered}
$$

where $T_{k}$ is the starting condition for the time step, $T_{k, l}$ is the intermediate result of the $l^{\text {th }}$ rule, the $\mathcal{F}_{l}$ 's are the rules, and $T_{k+1}$ is the final result, advancing the solution by one time step. The above is graphically rendered in Figure 4.2, using the same notation. This graphic shows both how the data feeds internal to a single time step, and how each time step feeds into the next. 


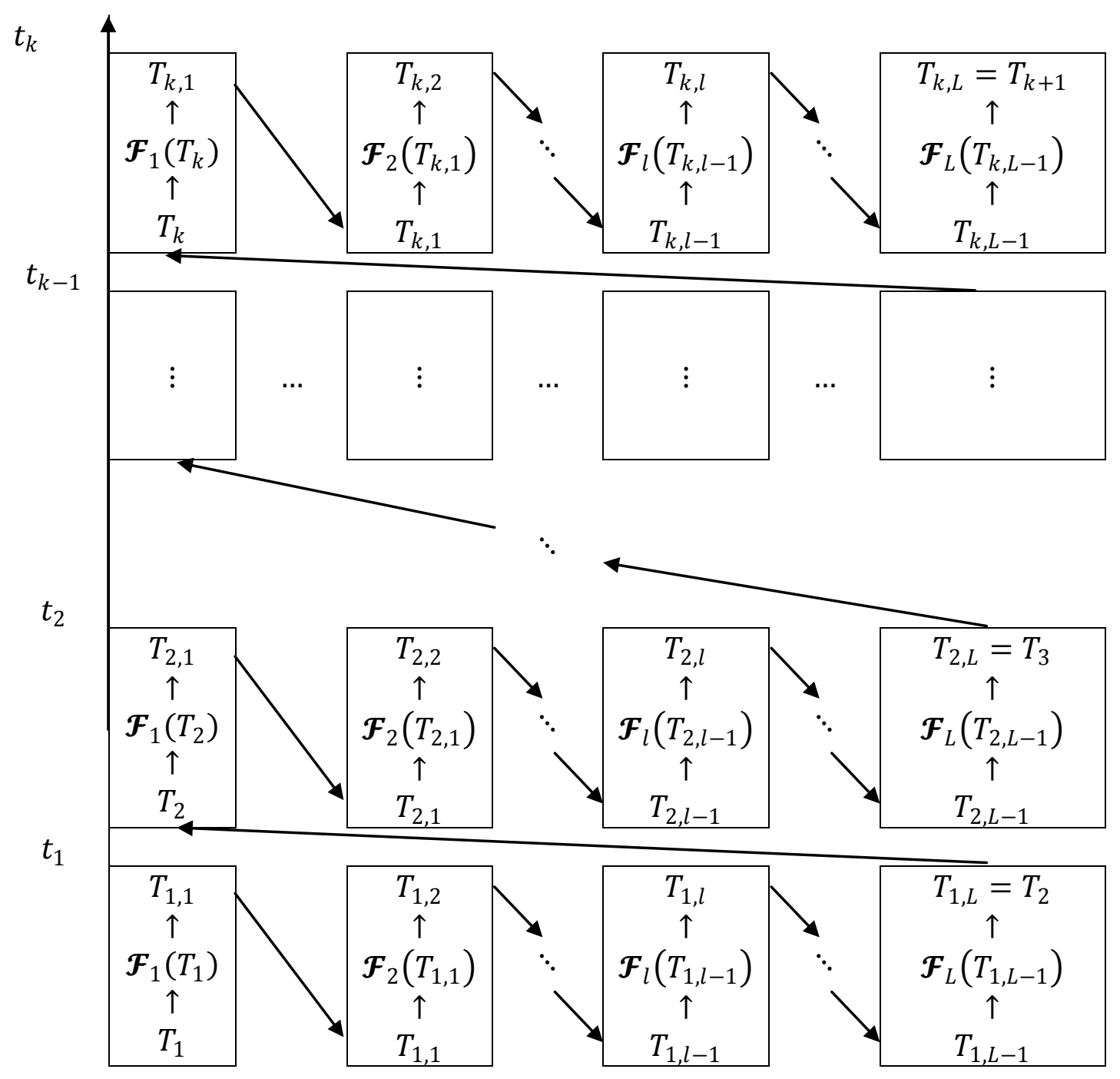

Figure 4.2: Graphical representation of the modified cellular automata feed-through.

A single step can be more concisely rendered mathematically as:

$$
T_{k+1}=\mathcal{F}_{L}\left(\mathcal{F}_{L-1}\left(\ldots \mathcal{F}_{l}\left(\ldots \mathcal{F}_{2}\left(\mathcal{F}_{1}\left(T_{k}\right)\right)\right)\right)\right)
$$

Then, to advance further, repeat the process over time.

For a given transport process, one will likely have an equation that includes diffusion, advection, proportional loss, and source terms. An example is the 1D, 1B process of section 3.4 which is: 


$$
\frac{\partial T}{\partial t}=\underbrace{\alpha \frac{\partial^{2} T}{\partial x^{2}}}_{\text {diffusion }}-\underbrace{V \frac{\partial T}{\partial x}}_{\text {advection }}-\underbrace{\frac{1}{\tau}\left(T-T_{\infty}\right)}_{\begin{array}{c}
\text { proportional } \\
\text { loss }
\end{array}}+\underbrace{}_{\begin{array}{c}
\text { source } \\
S_{c}
\end{array}}
$$

This equation can be broken down into three or four processes. These processes are:

$$
\begin{gathered}
\boldsymbol{f}_{a d v}\left(T_{k}\right)=-V \frac{\partial T}{\partial x} \\
\boldsymbol{f}_{\text {diff }}\left(T_{k}\right)=\alpha \frac{\partial^{2} T}{\partial x^{2}} \\
\boldsymbol{f}_{P L}\left(T_{k}\right)=-\frac{1}{\tau}\left(T-T_{\infty}\right) \\
\boldsymbol{f}_{\text {source }}\left(T_{k}\right)=S_{c}
\end{gathered}
$$

Because advective mechanics are simple when standalone, but difficult when mixed with other mechanics, the advection term can be separated out and handled very accurately with its own rule. This result is the primary reason to use this modified cellular automata technique. The diffusion mechanics, with the spatial grid shown above in Figure 4.1, is easily handled using a finite-difference solver. The proportional loss and source terms have analytical solutions, even when taken together. This allows the set of processes to be reduced to three.

The following sections discuss each of the individual rules, and their respective solution methods.

\subsection{Advection Rule}

The advection rule models the bulk transport behavior of the system. It is represented by the bulk transport term of the governing equation. It appears in the equation governing the moving body. The basic advection process with motion only in the ' $x$ ' direction is:

$$
\frac{\partial T}{\partial t}=-V \frac{\partial T}{\partial x}
$$

This particular piece of reduced physics can be solved analytically. The analytical solution is as follows:

$$
T(x, y, t)=T_{0}(x-V t, y)
$$

where $T_{0}(x, y)$ is the initial temperature distribution and $T(x, y, t)$ is the distribution at time equal to $t$. The interpretation of this result is that the initial distribution, under no other driving behaviors, simply shifts along the ' $x$ ' axis as time proceeds. This solution produces an 
extremely simple rule for a process that can be very difficult to deal with when mixed with other processes. This can be depicted as seen in Figure 4.3. It shows that, at each time step, that the shape does not change. The shape only translates as time progresses.

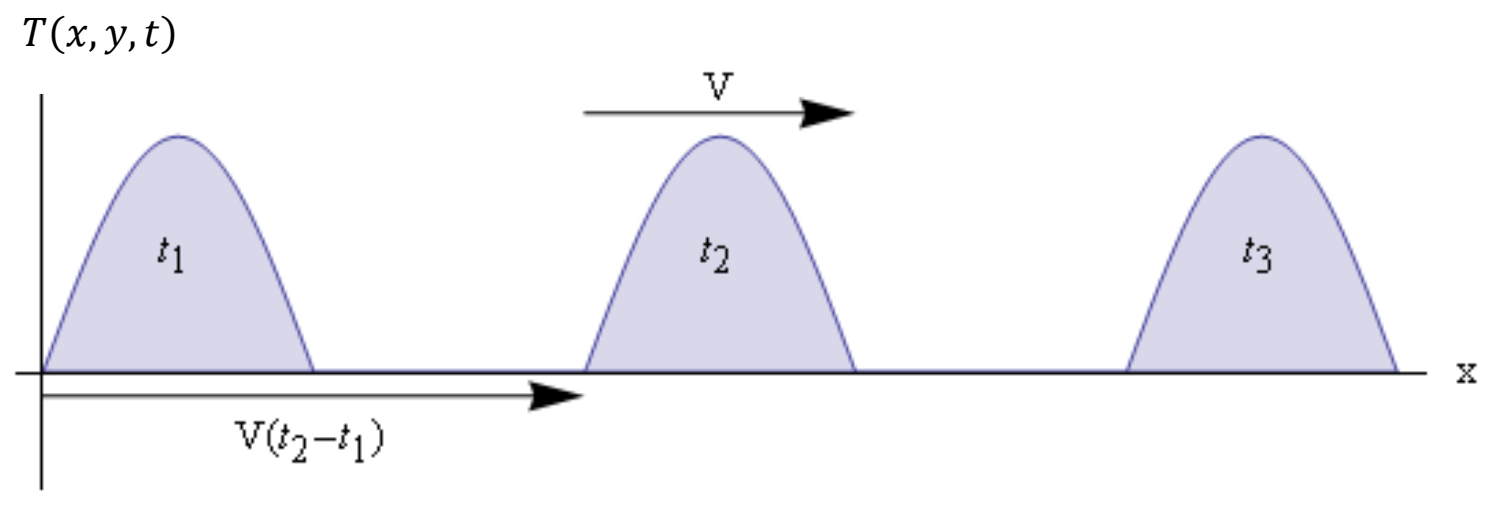

Figure 4.3: Graphical depiction of advection.

If the major time step $(\Delta t)$ over which each of the iterations of the automata is performed satisfies:

$$
\Delta t=\frac{N_{x} \Delta x}{V}
$$

then the solution advects by $N_{x}$ nodes. Thus, one can simply have the computer shift the solution (according to whatever bookkeeping method is used programmatically) to the next step, in an exact solution. As a result of this, the implementation of the direct solution uses this method to determine the major time step. Thus the rule is:

$$
T_{k+1}(x, y)=\mathcal{F}_{a d v}\left(T_{k}\right)=T_{k}\left(x-N_{x} \Delta x, y\right)
$$

\subsection{Source Partition Rule}

As far as the knowledge of the forcing functions in this example is concerned, what we know is the total heat flux distribution. However, the actual partition of heat into the two solids is not known. This lack of knowledge leads to the need to approximate how the interface partitions the heat. This partition is a function of the thermal properties and current temperature distributions of the solids.

As a result of the physical contact at the contact junctures, one must account for conduction at the interface, along with the energy imparted to the system by friction. 
Mathematically, this conduction term appears, but one must ensure that the conduction is removed if the contact distribution is zero. Thus, over regions where $f_{d}(x, t)>0$, the following boundary conditions apply:

$$
\begin{gathered}
q_{\text {fric }}^{\prime \prime}(x, t)=q_{\text {fric } 2}^{\prime \prime}(x, t)+q_{f r i c_{1}}^{\prime \prime}(x, t)=\left.k_{2} \frac{\partial T_{2}}{\partial y}\right|_{y=0}-\left.k_{1} \frac{\partial T_{1}}{\partial y}\right|_{y=0} \\
T_{2_{s}}=T_{1_{s}}=T_{s}
\end{gathered}
$$

These conditions state that the frictional energy goes into the two solids, and that the surface temperatures are equal at the contacts. This partition can be visualized in Figure 4.4.

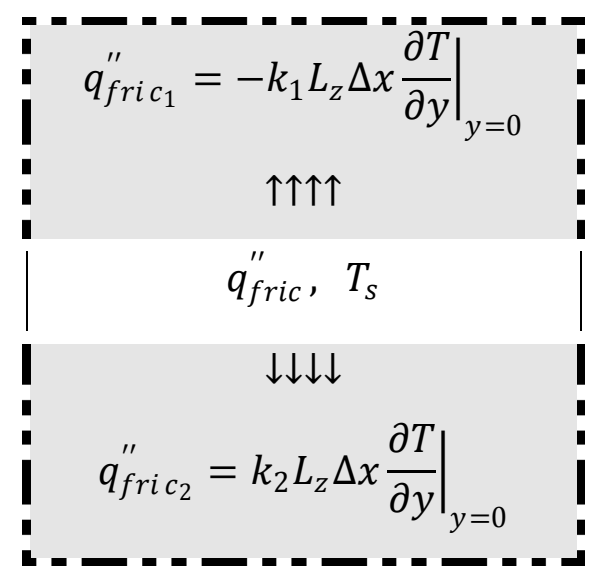

Figure 4.4: Visualization of the heat partition rule.

Approximating these interface conditions using a finite difference method, one gets:

$$
q_{\text {fric }}^{\prime \prime}(x, t)=\frac{2 k_{2}}{\Delta y_{2}}\left(T_{s}-T_{2}\right)-\frac{2 k_{1}}{\Delta y_{1}}\left(T_{1}-T_{s}\right)
$$

By rearranging this expression, one can determine the surface temperature:

$$
T_{s}=\frac{q_{\text {fric }}^{\prime \prime}(x, t)+\frac{2 k_{1}}{\Delta y_{1}} T_{1}+\frac{2 k_{2}}{\Delta y_{2}} T_{2}}{\frac{2 k_{1}}{\Delta y_{1}}+\frac{2 k_{2}}{\Delta y_{2}}}
$$

This equation is a very useful result that is used to determine the partition of heat, and can be used in post-computational analysis for further insight.

By substituting the surface temperature into the finite difference approximation of $q_{\text {fric }_{1}}^{\prime \prime}(x, t)$, one gets: 


$$
q_{\text {fric }}^{\prime \prime}=-\frac{2 k_{1}}{\Delta y_{1}}\left(T_{1}-\frac{q_{f r i c}^{\prime \prime}(x, t)+\frac{2 k_{1}}{\Delta y_{1}} T_{1}+\frac{2 k_{2}}{\Delta y_{2}} T_{2}}{\frac{2 k_{1}}{\Delta y_{1}}+\frac{2 k_{2}}{\Delta y_{2}}}\right)
$$

This equation can be rearranged to acquire a convenient expression for the heat flux into body 1 . Further, by using the definition of the total heat flux, one also readily attains the heat flux into body 2 .

$$
\begin{gathered}
q_{\text {fric } 1}^{\prime \prime}(x, t)=\frac{\frac{2 k_{1}}{\Delta y_{1}}}{\frac{2 k_{1}}{\Delta y_{1}}+\frac{2 k_{2}}{\Delta y_{2}}}\left(q_{\text {fric }}^{\prime \prime}(x, t)+\frac{2 k_{2}}{\Delta y_{2}}\left(T_{2}-T_{1}\right)\right) \\
q_{\text {fri } c_{2}}^{\prime \prime}(x, t)=q_{\text {fric }}^{\prime \prime}(x, t)-q_{\text {fric }}^{\prime \prime}(x, t)
\end{gathered}
$$

These results will eventually become the source term in an analytical solution for the effect of the boundary and source terms.

Over domains where the contact distribution is zero, the heat flux into the two bodies must be zero:

$$
\begin{aligned}
& q_{{\text {fri } c_{1}}^{\prime \prime}(x, t)}=0 \\
& q_{{\text {fri } c_{2}}^{\prime \prime}(x, t)}^{\prime \prime}=0
\end{aligned}
$$

These results, with the temperatures (as needed) evaluated at $t_{k}$, give a roughly constant source for a classic Newtonian cooling solution below.

\subsection{Convection plus Source Rule}

The formulation of the convective loss and source depends on whether the model is one or two dimensional. The two dimensional process must be broken up into regions. The convection process only occurs on surface nodes outside the contact area while heat addition only occurs inside the contact region.

\subsubsection{Two Dimensional Process}

For surface nodes that are contained inside the contact region, the governing process is:

$$
\rho c \Delta x \Delta y L_{z} \frac{\partial T_{i}}{\partial t}=\Delta x L_{z} \cdot q_{\text {fri } c_{b o d y}}^{\prime \prime}\left(x_{i}, t\right)
$$

where $q_{\text {fric } c_{b o d y}}^{\prime \prime}\left(x_{i}, t\right)$ is the frictional heat that enters the node. The subscript 'body' simply indicates that it is the appropriate part of the heat partition, e.g., that if node $i$ is contained in 
Body 1 , then it would be $q_{\text {fric }}^{\prime \prime}\left(x_{i}, t\right)$. If the source behaves as a constant for each major time step (it can change from time step to time step), then the equation becomes:

$$
\frac{\partial T_{i}}{\partial t}=\frac{q_{\text {fri } c_{b o d y}}^{\prime \prime}\left(x_{i}\right)}{\rho c \Delta y}
$$

Conveniently, one can substitute $S_{c_{i}}=\frac{q_{f r i c}^{\prime \prime}{ }_{b o d y}\left(x_{i}\right)}{\rho c \Delta y}$ into the process and gain the convenient representation of the process as:

$$
\frac{\partial T_{i}}{\partial t}=S_{c_{i}}
$$

This is easily integrated to create the rule for these nodes:

$$
T_{k+1}=\mathcal{F}_{2 \text { source }}\left(T_{k}\right)=S_{c} \Delta t+T_{k}
$$

For the nodes on the surfaces that are parallel to the contact surface outside the nominal contact region, the governing process is:

$$
\rho c \Delta x \Delta y L_{z} \frac{\partial T_{i}}{\partial t}=-h \Delta x L_{z}\left(T_{i}-T_{\infty}\right)
$$

Through algebraic manipulation, and with the substitution of $\tau=\frac{\rho c \Delta y}{h}$, the process can be represented as:

$$
\frac{\partial T_{i}}{\partial t}=-\frac{\left(T_{i}-T_{\infty}\right)}{\tau}
$$

This is easily integrated to give the rule for this process:

$$
T_{k+1}=\mathcal{F}_{2 D \text { conv }}\left(T_{k}\right)=T_{\infty}+\left(T_{k}-T_{\infty}\right) \exp \left(-\frac{\Delta t}{\tau}\right)
$$

This is the classic result of a lumped capacity, Newtonian cooling heat transfer problem. This is the result one would expect. For the nodes that are on the surfaces that are perpendicular to the contact surface, with a different time constant of $\tau=\frac{\rho c \Delta x}{h}$, the same result is obtained.

\subsubsection{One Dimensional Process}

For the nodes that are contained within the contact region, the governing process is:

$$
\rho c \Delta x L_{y_{b o d y}} L_{z} \frac{\partial T_{i}}{\partial t}=-h \Delta x L_{z}\left(T_{i}-T_{\infty}\right)+\Delta x L_{z} \cdot q_{\text {fric body }}^{\prime \prime}\left(x_{i}, t\right)
$$

again, where $q_{\text {fric body }}^{\prime \prime}\left(x_{i}, t\right)$ is the frictional heat that enters the node. If the source is again treated as a constant for each major time step, then the equation becomes: 


$$
\frac{\partial T_{i}}{\partial t}=-\frac{h}{\rho c L_{y_{b o d y}}}\left(T_{i}-T_{\infty}\right)+\frac{q_{f r i c_{b o d y}}^{\prime \prime}\left(x_{i}\right)}{\rho c L_{y_{b o d y}}}
$$

In a fashion similar to the two dimensional development, one can make the convenient, choice of substitutions:

$$
\begin{gathered}
S_{c_{i}}=\frac{q_{\text {fri } c_{\text {body }}}^{\prime \prime}\left(x_{i}\right)}{\rho c L_{y_{b o d y}}} \\
\tau=\frac{\rho c L_{y_{b o d y}}}{h}
\end{gathered}
$$

This substitution gives the representation of the process in the contact region as:

$$
\frac{\partial T_{i}}{\partial t}=-\frac{\left(T_{i}-T_{\infty}\right)}{\tau}+S_{c_{i}}
$$

This equation has an analytical solution as a simple rule for this process as:

$$
T_{k+1}=\mathcal{F}_{1 \text { ssource }}\left(T_{k}\right)=T_{\infty}+\tau S_{c}+\left(T_{k}-T_{\infty}-\tau S_{c}\right) \exp \left(-\frac{\Delta t}{\tau}\right)
$$

Outside the contact region, the process changes to:

$$
\frac{\partial T_{i}}{\partial t}=-\frac{2 h}{\rho c L_{y_{b o d y}}}\left(T_{i}-T_{\infty}\right)
$$

And, by using the same substitution for the time constant above, the rule is:

$$
T_{k+1}=\mathcal{F}_{1 \text { Dconv }}\left(T_{k}\right)=T_{\infty}+\left(T_{k}-T_{\infty}\right) \exp \left(-\frac{2 \Delta t}{\tau}\right)
$$

\subsection{Diffusion Rule}

The diffusion process models the heat conduction that occurs in the system. It appears in the governing equations for both bodies as:

$$
\frac{\partial T}{\partial t}=\alpha\left(\frac{\partial^{2} T}{\partial x^{2}}+\frac{\partial^{2} T}{\partial y^{2}}\right)
$$

By recalling the discrete spatial grid from Figure 4.1 that was created for the numerical model, one can reformulate the above equation from a partial differential equation into a system of ordinary differential equations. The formulation mathematically appears to be discrete in space and continuous in time. The formulation follows as:

$$
\frac{d T_{i, j}}{d t}=\alpha\left(\frac{T_{i+1, j}-2 T_{i, j}+T_{i-1, j}}{\Delta x^{2}}+\frac{T_{i, j+1}-2 T_{i, j}+T_{i, j-1}}{\Delta y^{2}}\right)
$$


where the subscripts $i$ and $j$ indicate the nodal $(x, y)$ index. Properly handled, this particular formulation can be broken down into two smaller rules, where one performs the conduction in one direction first, and then the other. This method then enables the use of a tridiagonal solver in an implicit finite difference scheme, which is computationally very quick. Thus, the rule formulation becomes:

$$
\frac{T_{i}^{k+1}-T_{i}^{k}}{\Delta t}=\alpha\left(\frac{T_{i+1}^{k+1}-2 T_{i}^{k+1}+T_{i-1}^{k+1}}{\Delta x^{2}}\right)
$$

This equation is readily arranged into a tridiagonal matrix, $\boldsymbol{A}$, with upper diagonal $\boldsymbol{a}_{u}$, lower diagonal $\boldsymbol{a}_{l}$, and main diagonal $\boldsymbol{a}_{d}$. This can then be employed in the matrix equation:

$$
\boldsymbol{A} \boldsymbol{T}^{k+1}=\boldsymbol{T}^{k}
$$

This equation is ready for the computer to solve using a tridiagonal solver solver as the solution rule. Thus, the rule is:

$$
T_{k+1}=\mathcal{F}_{\text {diff }}\left(T_{k}\right)=\operatorname{tridiagonal}\left(\boldsymbol{a}_{u}, \boldsymbol{a}_{d}, \boldsymbol{a}_{l}, T_{k}\right)
$$

where the tridiagonal $\left(\quad\right.$ ) operator is the tridiagonal solver, the $\boldsymbol{a}$ 's are the diagonals, and $T_{k}$ is the initialization of the solver.

\subsection{Rule Library}

In summation therefore, the rules are:

- $T_{k+1}=\mathcal{F}_{a d v}\left(T_{k}\right)=T_{k}\left(x-N_{x} \Delta x, y\right)$

- $T_{k+1}=\mathcal{F}_{2 D \text { source }}\left(T_{k}\right)=S_{c} \Delta t+T_{k}$

- $T_{k+1}=\mathcal{F}_{2 D \operatorname{conv}}\left(T_{k}\right)=T_{\infty}+\left(T_{k}-T_{\infty}\right) \exp \left(-\frac{\Delta t}{\tau}\right)$

- $T_{k+1}=\mathcal{F}_{1 \text { Dsource }}\left(T_{k}\right)=T_{\infty}+\tau S_{c}+\left(T_{k}-T_{\infty}-\tau S_{c}\right) \exp \left(-\frac{\Delta t}{\tau}\right)$

- $T_{k+1}=\mathcal{F}_{1 D c o n v}\left(T_{k}\right)=T_{\infty}+\left(T_{k}-T_{\infty}\right) \exp \left(-\frac{2 \Delta t}{\tau}\right)$

- $T_{k+1}=\mathcal{F}_{\text {diff }}\left(T_{k}\right)=\operatorname{tridiagonal}\left(\boldsymbol{a}_{u}, \boldsymbol{a}_{d}, \boldsymbol{a}_{l}, T_{k}\right)$

These rules can be mixed and matched to model any combination of these effects. Also, additional rules for other physical effects can be developed and added to the library of rules for modeling more complex processes. 


\section{Estimation of Real Area of Contact}

The estimation of the real area of contact requires the use of methods developed to solve inverse mathematical problems. While there are many inverse problem methods, the scheme used here is the Levenberg-Marquardt Method.

\subsection{Algorithm}

Parameter estimation problems are a special case of the inverse problem, where the forcing functions are known, and the response is known to some degree (has been sampled by instrumentation), but some aspect of the system is not known. This is represented in the context of the research problem in Figure 5.1. The objective is to approximate the contact distribution.

\begin{tabular}{|c|c|c|c|c|c|c|c|c|c|c|}
\hline \multicolumn{2}{|c|}{ Forcing Function } & \multirow{3}{*}{$\rightarrow$} & \multicolumn{6}{|c|}{ System } & \multirow{3}{*}{$\rightarrow$} & Response \\
\hline$T_{0}$ & $q_{\text {fric }}$ & & $k^{\prime} s$ & $h^{\prime} s$ & $\alpha^{\prime} s$ & $L^{\prime} S$ & $f_{d}\left(x_{i}, t_{m}\right)$ & $V$ & & $\begin{array}{c}Y_{m} \\
m=1,2, \ldots, M\end{array}$ \\
\hline$\sqrt{ }$ & $\sqrt{ }$ & & $\sqrt{ }$ & $\sqrt{ }$ & $\sqrt{ }$ & $\sqrt{ }$ & $?$ & $\sqrt{ }$ & & $\sqrt{ }$ \\
\hline
\end{tabular}

Figure 5.1: Depiction of knowns and unknowns in the real area of contact estimation problem.

The addition of the measurement system appears below in Figure 5.2. The black dots show the conceptual locations of the measurements.

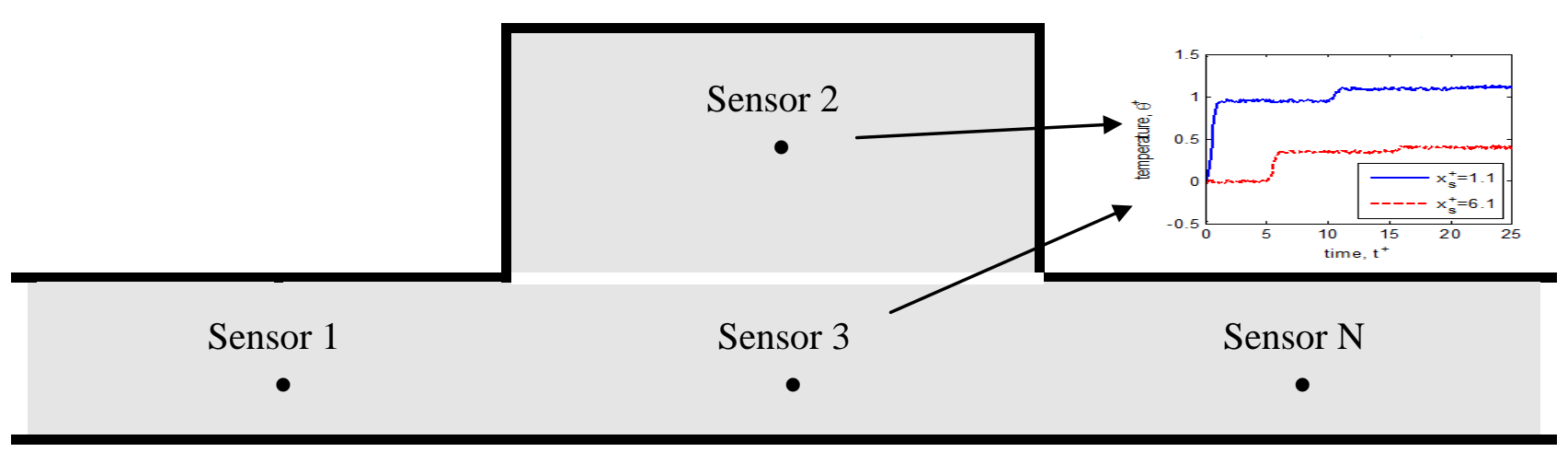

Figure 5.2: Conceptual layout of measurement locations. 
The response of the system would be measured experimentally and tabulated against its sample time and location for input into the computer (See Table 5.1).

Table 5.1: Sample table of data.

\begin{tabular}{|c|c|c|c|c|}
\hline time & Sensor 1 & Sensor 2 & $\ldots$ & Sensor $N_{s}$ \\
\hline$t_{1}$ & $Y_{1_{1}}$ & $Y_{2_{1}}$ & $\ldots$ & $Y_{N_{s 1}}$ \\
\hline$t_{2}$ & $Y_{1_{2}}$ & $Y_{2}$ & $\ldots$ & $Y_{N_{s 2}}$ \\
\hline$\vdots$ & $\vdots$ & $\vdots$ & $\ddots$ & $\vdots$ \\
\hline$t_{N_{t}}$ & $Y_{1_{N_{t}}}$ & $Y_{2_{N_{t}}}$ & $\ldots$ & $Y_{N_{S_{t}}}$ \\
\hline
\end{tabular}

Mathematically, parameter estimation is done by minimizing the sum of squares of the differences between the data and the model. Mathematically, the sum of squares is:

$$
S(\boldsymbol{\beta})=\sum_{m=1}^{M}\left(Y_{m}-\widehat{T}_{m}(\boldsymbol{\beta})\right)^{2}
$$

where $\boldsymbol{\beta}$ is the vector of unknown parameters, the $Y_{m}$ 's are the data, and the $\widehat{T}_{m}$ 's are the model values at the measurement times and locations for the current set of parameters. $M$ is the totality of all measurements. For the physical model presented here, the $\beta$ 's are the values of the contact distribution evaluated at each nodal location inside the nominal contact zone. To do this, one must find the bottom of the 'bowl' formed by the sum of squares as a function of all the parameters. Conceptually, the bowls appear in Figure 5.3. For one parameter systems, it looks like a dip in a line. For two parameter systems, it looks like a bowl.
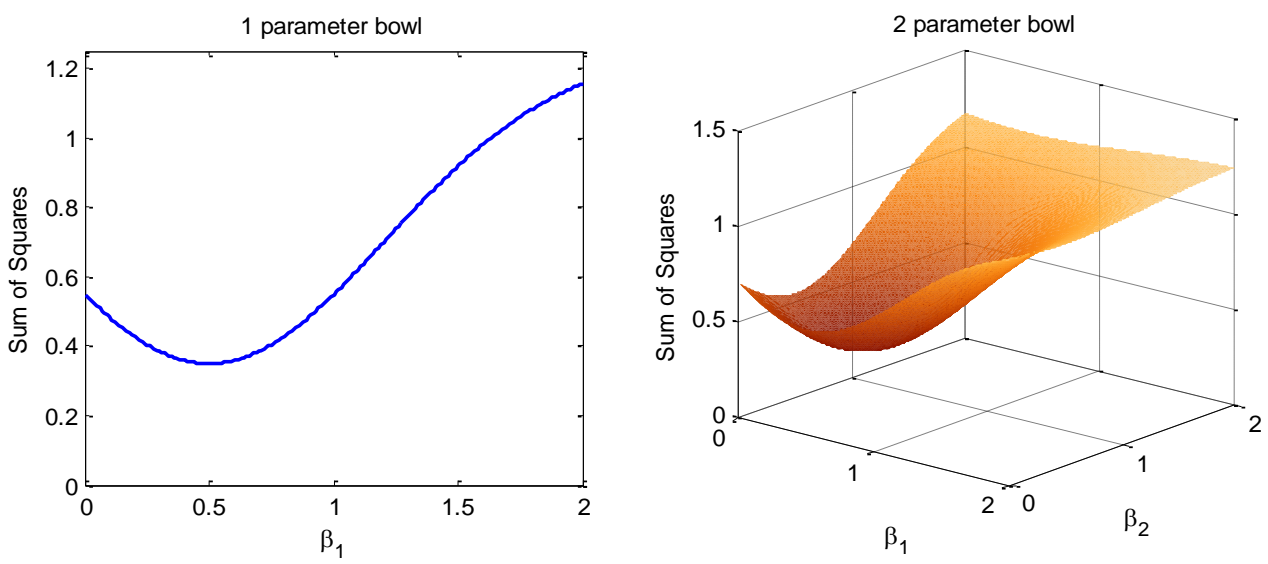

Figure 5.3: Conceptual representation of one and two parameter sums of squares. 
The bottom of the hyper-dimensional bowl is located where the derivative of the sum of squares with respect to each of the parameters is zero:

$$
\begin{gathered}
\frac{\partial S(\boldsymbol{\beta})}{\partial \beta_{n}}=-2 \sum_{m=1}^{M} \frac{\partial \widehat{T}_{m}(\boldsymbol{\beta})}{\partial \beta_{n}}\left(Y_{m}-\widehat{T}_{m}(\boldsymbol{\beta})\right)=0 \\
n=1,2, \ldots, N_{\text {con }}
\end{gathered}
$$

The derivative of the model with respect to the various parameters, when in matrix form, is called the sensitivity matrix, $\boldsymbol{X}$. The vector of differences is the residuals, $\boldsymbol{R}$. These are:

$$
\begin{gathered}
\boldsymbol{X}(\boldsymbol{\beta})=\left[\begin{array}{ccc}
\frac{\partial \widehat{T}_{1}}{\partial \beta_{1}} & \cdots & \frac{\partial \widehat{T}_{1}}{\partial \beta_{N}} \\
\vdots & \ddots & \vdots \\
\frac{\partial \widehat{T}_{M}}{\partial \beta_{1}} & \cdots & \frac{\partial \widehat{T}_{M}}{\partial \beta_{N}}
\end{array}\right] \\
\boldsymbol{R}(\boldsymbol{\beta})=\boldsymbol{Y}-\widehat{\boldsymbol{T}}(\boldsymbol{\beta})=\left[\begin{array}{c}
Y_{1}-\widehat{T}_{1}(\boldsymbol{\beta}) \\
\vdots \\
Y_{M}-\widehat{T}_{M}(\boldsymbol{\beta})
\end{array}\right]
\end{gathered}
$$

Using this matrix notation, Equation 5.2 becomes:

$$
\boldsymbol{X}(\boldsymbol{\beta})^{T} \boldsymbol{R}(\boldsymbol{\beta})=\mathbf{0}
$$

Because many of these parameters affect the system nonlinearly, one must use one of many iterative methods to solve this equation for the unknown parameters, $\boldsymbol{\beta}$. These methods vary widely, but as a means of keeping the mathematics as physical as possible, while requiring some numerical stability, the Levenberg-Marquardt method is used [27]. Most least-squares methods are based on the Gauss method. The Gauss method is a linearized iterative technique, or a first order Taylor series. The Taylor series is developed as follows:

$$
\left.\boldsymbol{T}(\boldsymbol{\beta})=\sum_{\xi=0}^{\Xi} \frac{\partial^{\xi} \widehat{\boldsymbol{T}}(\boldsymbol{\beta})}{\partial \boldsymbol{\beta}^{\xi}}\right)_{\boldsymbol{\beta}_{i t e r}} \frac{\left(\boldsymbol{\beta}-\boldsymbol{\beta}_{\text {iter }}\right)^{\xi}}{\xi !}
$$

which, in its first order form expands to:

$$
\left.\boldsymbol{T}(\boldsymbol{\beta})=\widehat{\boldsymbol{T}}\left(\boldsymbol{\beta}_{\text {iter }}\right)+\frac{\partial \widehat{\boldsymbol{T}}(\boldsymbol{\beta})}{\partial \boldsymbol{\beta}}\right)_{\boldsymbol{\beta}_{\text {iter }}}\left(\boldsymbol{\beta}-\boldsymbol{\beta}_{\text {iter }}\right)
$$

In order to gain the value of the parameters for the next step in the iteration, this can be substituted into Equation 5.5, with the derivative substituted to form: 


$$
\boldsymbol{X}\left(\boldsymbol{\beta}_{i t e r}\right)^{T}\left(\boldsymbol{Y}-\left(\widehat{\boldsymbol{T}}\left(\boldsymbol{\beta}_{\text {iter }}\right)+\boldsymbol{X}\left(\boldsymbol{\beta}_{\text {iter }}\right)\left(\boldsymbol{\beta}_{\text {iter }+1}-\boldsymbol{\beta}_{\text {iter }}\right)\right)\right)=\mathbf{0}
$$

This equation can be rearranged to compute the next step in the iteration for $\boldsymbol{\beta}_{\text {iter }+1}$ :

$$
\boldsymbol{\beta}_{\text {iter }+1}=\boldsymbol{\beta}_{\text {iter }}+\left[\boldsymbol{X}_{\text {iter }}^{T} \boldsymbol{X}_{\text {iter }}\right]^{-1} \boldsymbol{X}_{\text {iter }}^{T} \boldsymbol{R}\left(\boldsymbol{\beta}_{\text {iter }}\right)
$$

The Gauss method has no enforced numerical stability, and thus, for ill conditioned sensitivity matrices $\left(\left|\boldsymbol{X}^{T} \boldsymbol{X}\right| \cong 0\right)$, this equation can become quite unstable. In order to correct this problem, the diagonal of this matrix must be strengthened. This strengthening is done by adding a damping parameter to the diagonal as seen below.

$$
\boldsymbol{\beta}_{\text {iter }+1}=\boldsymbol{\beta}_{\text {iter }}+\left[\boldsymbol{X}_{\text {iter }}^{T} \boldsymbol{X}_{\text {iter }}+\mu_{\text {iter }} \boldsymbol{\Omega}_{\text {iter }}\right]^{-1} \boldsymbol{X}_{\text {iter }}^{T} \boldsymbol{R}\left(\boldsymbol{\beta}_{\text {iter }}\right)
$$

As the solution converges, the Levenberg-Marquardt method reduces the damping parameter $\mu_{i t e r}$ until convergence. At convergence, the solution is very nearly the Gauss method, because $\mu_{i t e r}$ is very nearly zero. The choice of the diagonal matrix $\boldsymbol{\Omega}_{i t e r}$ varies. For convenience, the identity matrix is used [27]. As a matter of computational simplicity, if the model is linear in a given parameter, the sensitivity matrix only needs to be computed once. While, for a given linear set of equations, one can directly solve for the least squares values of the parameters, it can be very difficult if the matrix is weak on the diagonal. As a result, this Levenberg-Marquardt method can still be utilized to solve this problem. This technique is the approach used in the solutions presented in chapter 6.

For each iteration, one must compute a new value for the damping parameter. To do this, one must first advance the solution to the iter +1 state. Then, determine if the sum of squares has decreased. If it has not, increase the damping parameter by one order of magnitude and try again, discarding the new $\boldsymbol{\beta}_{\text {iter }+1}$, and trying again. If the sum of squares did decrease, retain the new $\boldsymbol{\beta}_{\text {iter }+1}$ as $\boldsymbol{\beta}_{\text {iter }}$, and decrease the damping parameter by one order of magnitude. Repeat this procedure until an appropriate stopping criterion is met. For the purposes of this numerical experiment, it will be when the sum of squares is reduced to an acceptable level. These criteria are expressed below:

$$
\begin{aligned}
& S\left(\boldsymbol{\beta}_{i t e r+1}\right) \geq S\left(\boldsymbol{\beta}_{i t e r}\right) \rightarrow \text { reject } \boldsymbol{\beta}_{i t e r+1}, \mu_{i t e r+1}=10 \mu_{i t e r} \\
& S\left(\boldsymbol{\beta}_{\text {iter }+1}\right)<S\left(\boldsymbol{\beta}_{\text {iter }}\right) \rightarrow \text { accept } \boldsymbol{\beta}_{i t e r+1}, \mu_{i t e r+1}=.1 \mu_{i t e r}
\end{aligned}
$$

The algorithm can be expressed conveniently as a flowchart. The fundamental flows are seen below in Figure 5.4. 


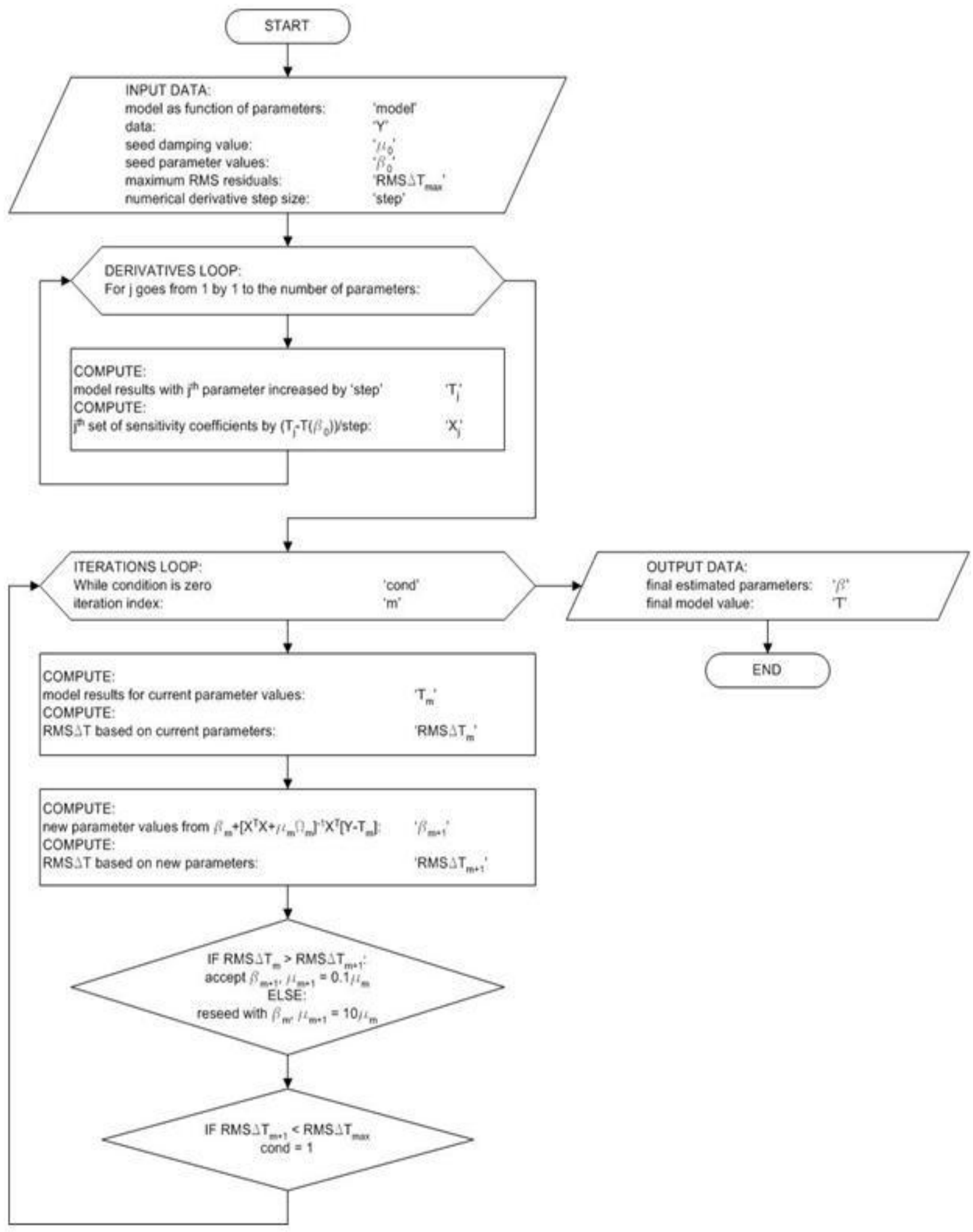

Figure 5.4: Parameter estimation algorithm flowchart. 
Upon completion of the procedure detailed above, the results are then ready for further analysis and interpretation.

\subsection{Application to Real Area of Contact}

As mentioned in Chapter 3, the estimation of the real area of contact is done by inference from the contact distribution. That requires the estimation method to determine what that distribution is. Since the model is discretized in space, only the mean value of the contact distribution over the length of each node needs to be determined. Thus the number of parameters that the model contains is equal to the number of nodes contained within the contact region. This discretization can be seen in Figure 5.5. Each node has its own value that can be estimated.

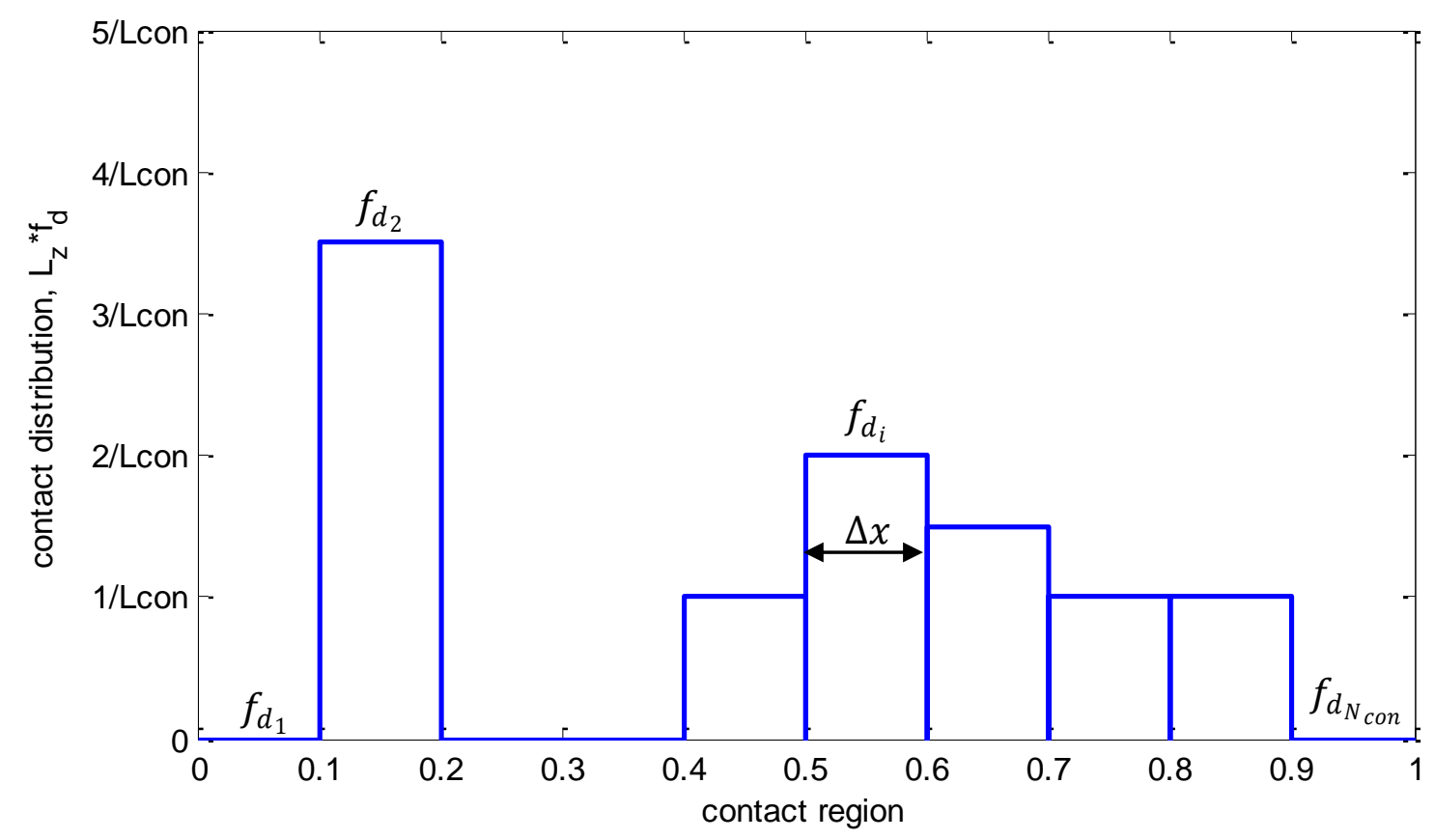

Figure 5.5: Arbitrary contact distribution discretized for estimation.

Upon estimating the contact distribution, various post-processing methods can be employed to determine the number and extent of each real contact. The sum of the sizes of each contact is the estimated contact area. The resolution on the estimation of the contact area is limited to the nodal sizes. In other words, the minimum contact area is limited to the area of a single node. 


\section{Results and Discussion}

This section details the findings of the parameter estimation study as the complexity of the models increases. The values to be estimated are the values of the discretized contact distribution as described in section 5.2. Prior to that, however, the means by which the solutions are simplified and normalized is covered. First, the equations for the 2D, $2 \mathrm{~B}$ system are normalized. This provides the normalizing variable groups that will be used throughout the results. Then, this section will cover how a high Peclet number changes the model.

\subsection{Non-Dimensionalization of the 2D, 2B Equations}

The set of equations and conditions in Table 3.1 must be non-dimensionalized in order to present generalized results. First, the contact distribution must be made dimensionless. Since, as was discussed in chapter 3 , the contact distribution has dimensions of $\frac{1}{L^{2}}$, we should normalize the distribution by substituting:

$$
q_{\text {fric }} \cdot f_{d}(x)=\frac{q_{\text {fric }}}{L_{z} L_{\text {con }}} L_{z} L_{\text {con }} f_{d}(x)=\frac{q_{\text {fric }}}{L_{z} L_{\text {con }}} f_{d}^{+}\left(x^{+}\right)
$$

where values denoted with a superscript plus are dimensionless. As part of this process, the ' $x$ ' location is referenced to the start of the contact for convenience in the development of the results, as opposed to the centered coordinates used for ease visualization in the formulation. Further substituting this into the equations in Table 3.1 results in this condition (with a characteristic length to be selected later):

$$
f_{d}^{+}\left(x^{+}\right)=\left\{\begin{array}{lr}
f^{+}\left(x^{+}\right), & x^{+} \leq \frac{L_{\text {con }}}{L_{\text {char }}} \\
0, & x^{+}>\frac{L_{\text {con }}}{L_{\text {char }}}
\end{array}\right.
$$

where $x^{+}=\frac{x-\frac{L_{c o n}}{2}}{L_{c h a r}}$. Now, the set of equations and conditions is ready to undergo its normalization. First, a list of independent variables, dependent variables, parameters, and parameter groups is important. This list is presented below in Table 6.1. 
Table 6.1: List of variables and parameters.

\begin{tabular}{|c|c|c|}
\hline \multicolumn{3}{|c|}{ Dependent Variable(s): } \\
\hline Temperature (static body) & $\theta_{1}$ & $K$ \\
\hline Temperature (moving body) & $\theta_{2}$ & $K$ \\
\hline \multicolumn{3}{|c|}{ Independent Variable(s): } \\
\hline Time & $t$ & $s$ \\
\hline Position in $\mathrm{x}$ & $x-\frac{L_{c o n}}{2}$ & $m$ \\
\hline Position in $\mathrm{y}$ & $y$ & $m$ \\
\hline \multicolumn{3}{|c|}{ Parameter(s): } \\
\hline Velocity & $V$ & $\frac{m}{s}$ \\
\hline Thermal diffusivity (body 1) & $\alpha_{1}$ & $\frac{m^{2}}{s}$ \\
\hline Thermal diffusivity (body 2) & $\alpha_{2}$ & $\frac{m^{2}}{s}$ \\
\hline Convective parameter (body 1 ) & $\frac{h_{1}}{\rho_{1} c_{1}}$ & $\frac{m}{s}$ \\
\hline Convective parameter (body 2) & $\frac{h_{2}}{\rho_{2} c_{2}}$ & $\frac{m}{s}$ \\
\hline Temperature rate (bulk, body 2) & $\frac{q_{\text {fric }}}{\rho_{2} c_{2} L_{y_{2}} L_{z} L_{c o n}}$ & $\frac{K}{s}$ \\
\hline Contact length & $L_{c o n}$ & $m$ \\
\hline Length in ' $x$ ' (body 2) & $L_{x}$ & $m$ \\
\hline Length in ' $y$ ' (body 1) & $L_{y_{1}}$ & $m$ \\
\hline Length in ' $y$ ' (body 2) & $L_{y_{2}}$ & $m$ \\
\hline
\end{tabular}

Once the list is prepared, then the number of units is determined. Here, there are three: degrees Kelvin, meters, and seconds. Thus, three independent parameters/parameter groups need to be selected for the normalization process. The selected groups are presented in Table 6.2. 
Table 6.2: Selected independent reference parameters.

\begin{tabular}{|l|c|c|}
\hline Velocity & $V$ & $\frac{m}{s}$ \\
\hline Contact length & $L_{c o n}$ & $m$ \\
\hline Temperature rate (bulk, body 2) & $\frac{q_{\text {fric }}}{\rho_{2} c_{2} L_{y_{2}} L_{z} L_{c o n}}$ & $\frac{K}{s}$ \\
\hline
\end{tabular}

For each of the units, the combination of parameters must be determined. This is done by setting a characteristic value equal to the product of the parameters each raised to a power. The combination of these parameters must result in only one dimension. The characteristic length is determined below in Table 6.3.

Table 6.3: Determination of charateristic length.

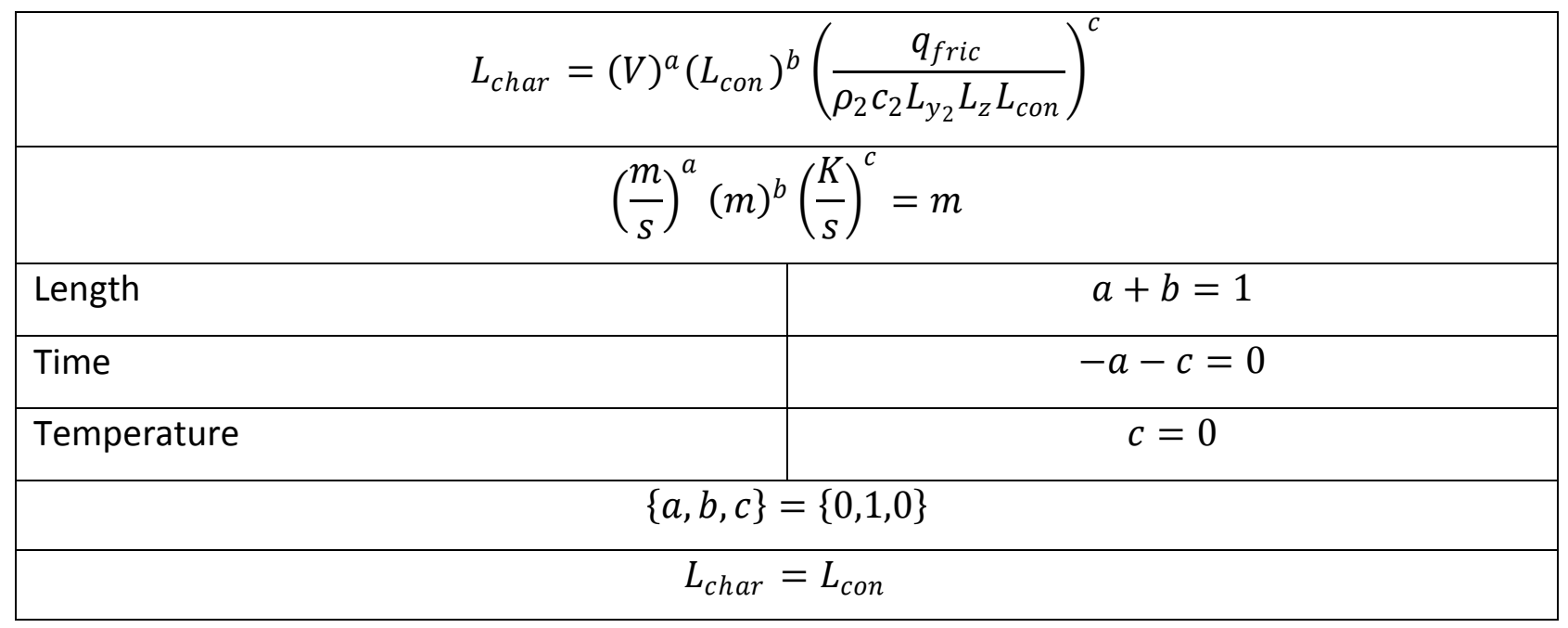

The characteristic time is determined below in Table 6.4. 
Table 6.4: Determination of charateristic time.

\begin{tabular}{|c|c|c|}
\hline$t_{c h a r}=(V)^{a}\left(L_{c o n}\right)^{b}$ & $\left(\frac{q_{\text {fric }}}{\rho_{2} c_{2} L_{y_{2}} L_{z} L_{\text {con }}}\right)$ & \\
\hline$\left(\frac{m}{s}\right)^{a}(m)^{b}$ & $\left(\frac{K}{s}\right)^{c}=s$ & \\
\hline Length & & $a+b=0$ \\
\hline Time & & $-a-c=1$ \\
\hline Temperature & & $c=0$ \\
\hline $\multicolumn{3}{|c|}{a, b, c}=\{-1,1,0\}$ \\
\hline$t_{\text {char }}=-$ & $\frac{L_{\text {con }}}{V}$ & \\
\hline
\end{tabular}

The characteristic temperature rise is determined below in Table 6.5.

Table 6.5: Determination of characteristic temperature rise.

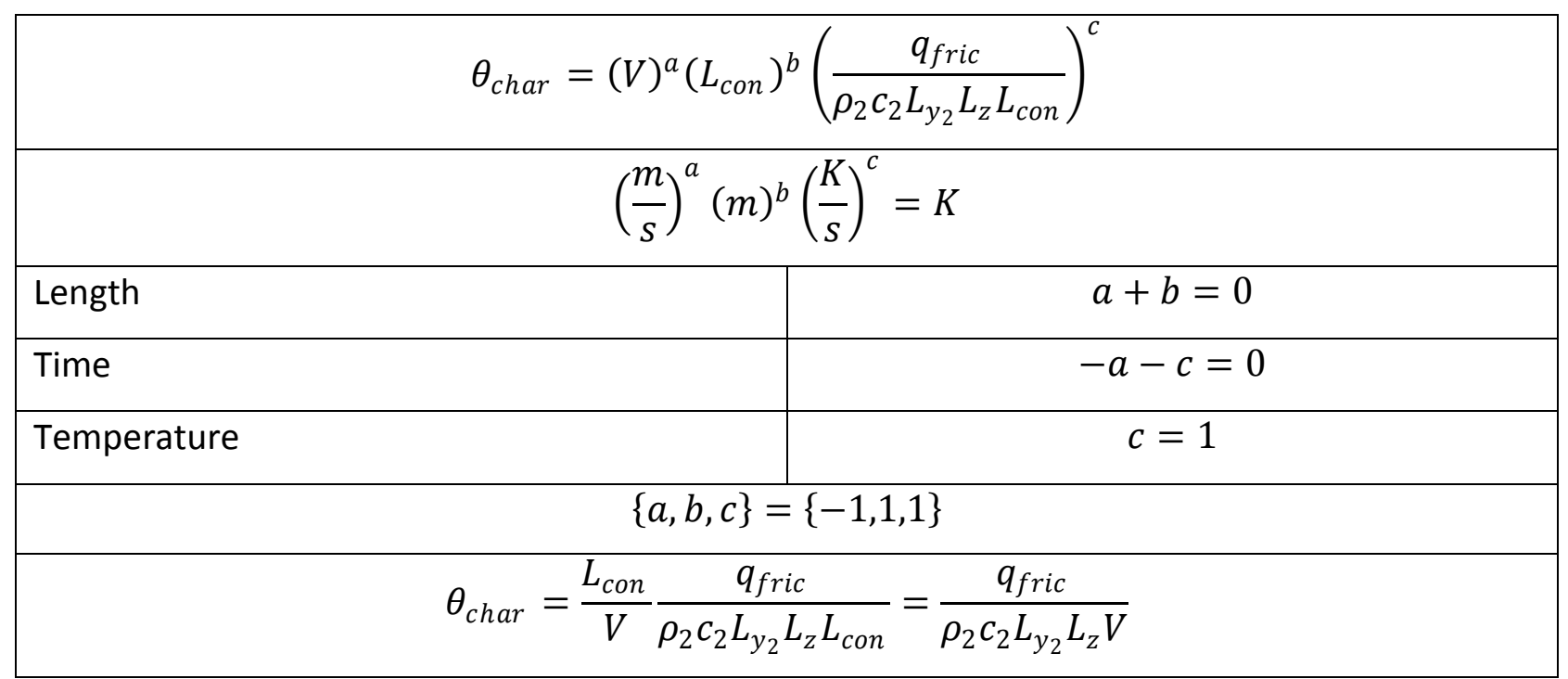

Below in Table 6.6 is a summary of the characteristic values that are used in the normalization of the equation. 
Table 6.6: Summary of characteristic values.

\begin{tabular}{|l|c|c|}
\hline Time & $t_{\text {char }}$ & $\frac{L_{c o n}}{V}$ \\
\hline Position & $L_{\text {char }}$ & $L_{c o n}$ \\
\hline Temperature & $\theta_{\text {char }}$ & $\frac{q_{\text {fric }}}{\rho_{2} c_{2} L_{y_{2}} L_{z} V}$ \\
\hline
\end{tabular}

Having determined the characteristic values, the dimensionless variables need to be determined. This is done by dividing the variable by its characteristic value. This is shown below in Table 6.7 .

Table 6.7: Dimensionless variables.

\begin{tabular}{|l|c|}
\hline Time & $t^{+}=\frac{t}{t_{\text {char }}}=\frac{t V}{L_{\text {con }}}$ \\
\hline Position & $x^{+}=\frac{x-\frac{L_{c o n}}{2}}{L_{\text {char }}}=\frac{x-\frac{L_{\text {con }}}{2}}{L_{\text {con }}}$ \\
\hline Temperature & $\theta^{+}=\frac{\theta}{\theta_{\text {char }}}=\frac{\theta \rho_{2} c_{2} L_{y_{2}} L_{z} V}{q_{\text {fric }}}$ \\
\hline
\end{tabular}

Since the dimensionless variables have been determined, the equation is now ready for normalization. The first step is to substitute the variables out for the dimensionless groups. This is demonstrated in Table 6.8 . 
Table 6.8: Normalization of Table 3.1.

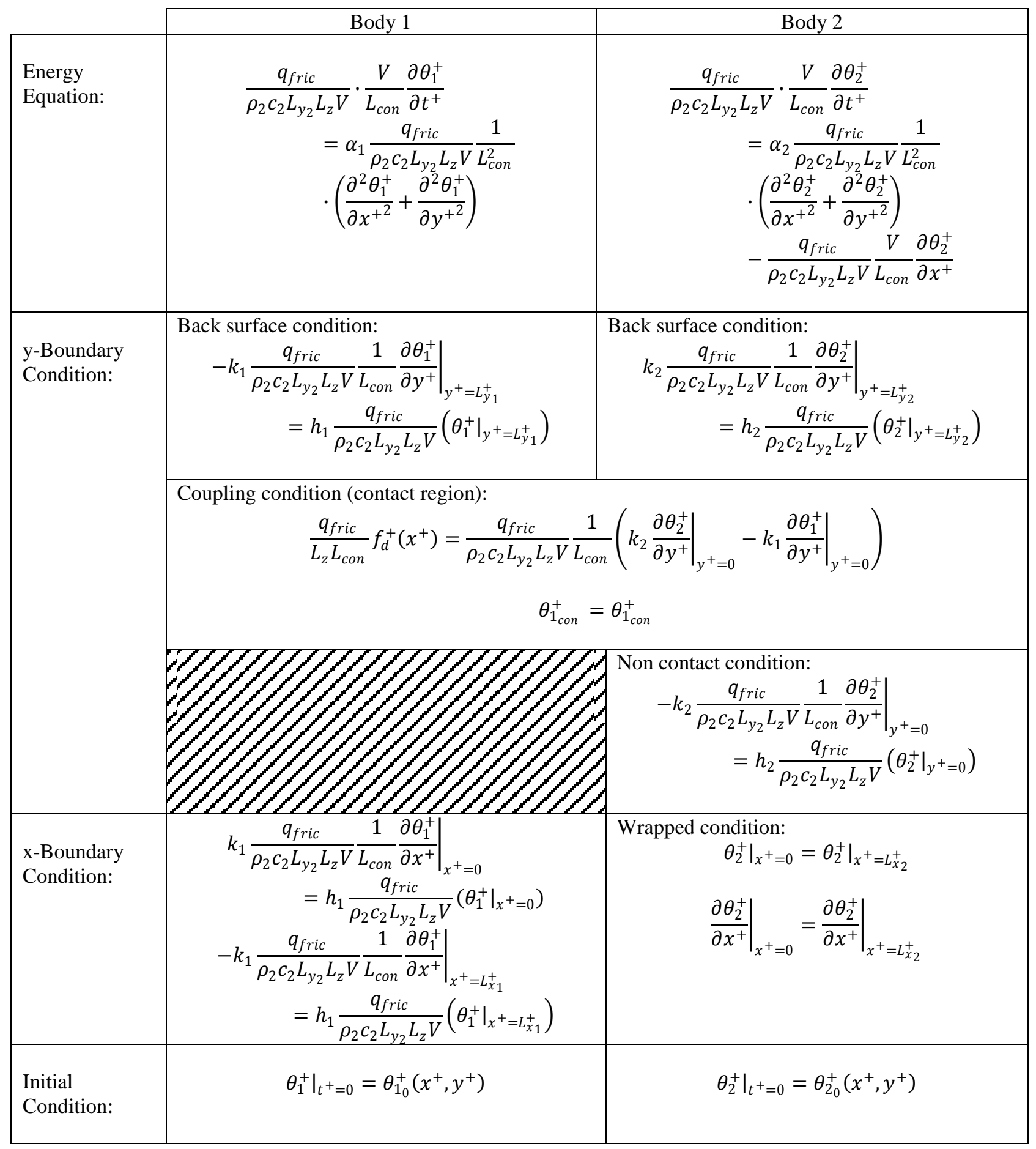

After manipulation, the table reduces to Table 6.9: 
Table 6.9: Normalized equations.

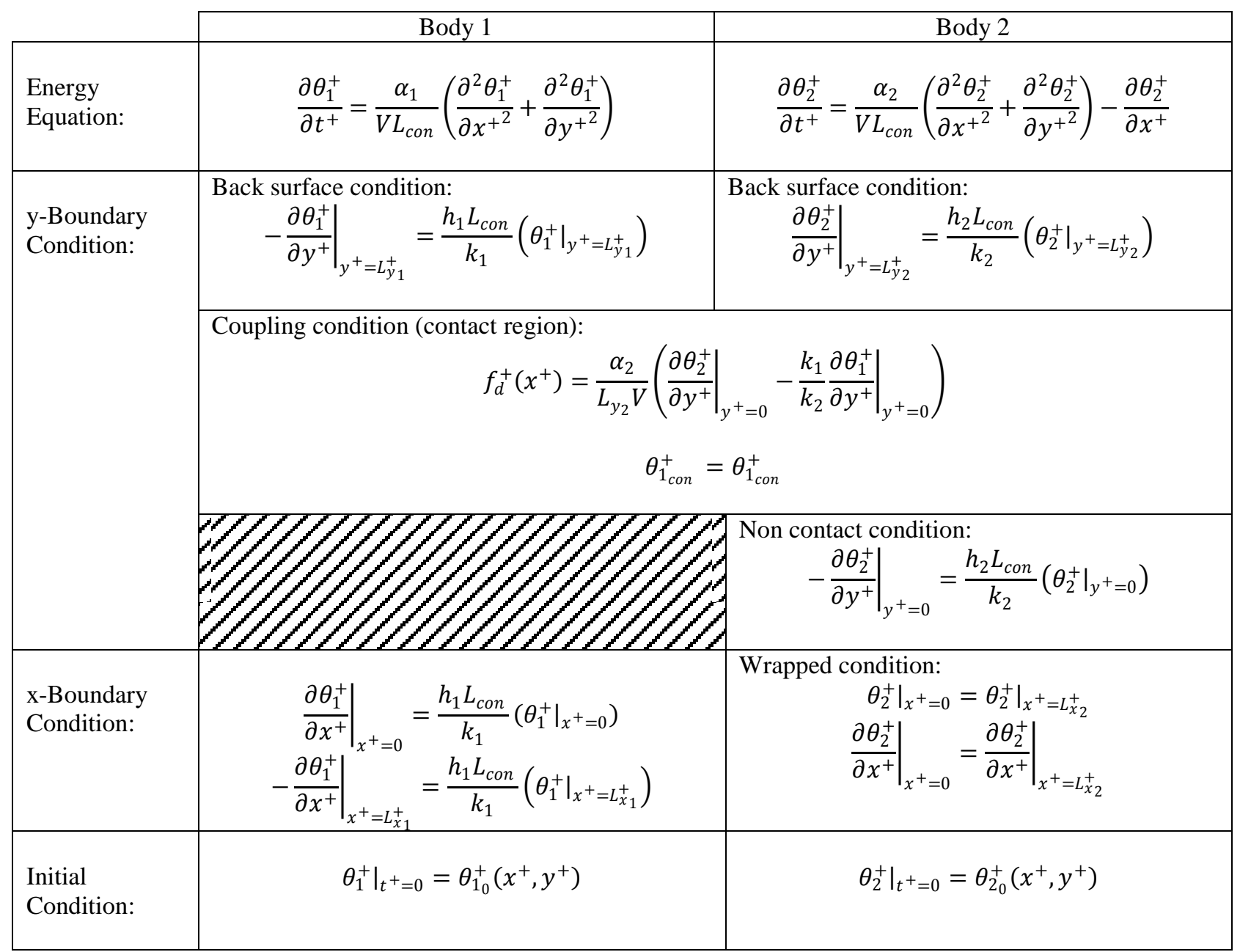

The Stanton and Peclet Numbers, along with parameter ratios as defined by:

$$
\begin{gathered}
S t=\frac{h_{2}}{\rho_{2} c_{2} V} \\
P e=\frac{V L_{c o n}}{\alpha_{2}} \\
k_{1}^{+}=\frac{k_{1}}{k_{2}} \\
h_{1}^{+}=\frac{h_{1}}{h_{2}} \\
\alpha_{1}^{+}=\frac{\alpha_{1}}{\alpha_{2}}
\end{gathered}
$$

can be substituted into the equation to result in Table 6.10: 
Table 6.10: Normalized 2D, 2B equations with dimensionless parameters.

\begin{tabular}{|c|c|c|}
\hline & Body 1 & Body 2 \\
\hline $\begin{array}{l}\text { Energy } \\
\text { Equation: }\end{array}$ & $\frac{\partial \theta_{1}^{+}}{\partial t^{+}}=\frac{\alpha_{1}^{+}}{P e}\left(\frac{\partial^{2} \theta_{1}^{+}}{\partial x^{+^{2}}}+\frac{\partial^{2} \theta_{1}^{+}}{\partial y^{+^{2}}}\right)$ & $\frac{\partial \theta_{2}^{+}}{\partial t^{+}}=\frac{1}{P e}\left(\frac{\partial^{2} \theta_{2}^{+}}{\partial x^{+^{2}}}+\frac{\partial^{2} \theta_{2}^{+}}{\partial y^{+^{2}}}\right)-\frac{\partial \theta_{2}^{+}}{\partial x^{+}}$ \\
\hline \multirow[t]{3}{*}{$\begin{array}{l}\text { y-Boundary } \\
\text { Condition: }\end{array}$} & $\begin{array}{l}\text { Back surface condition: } \\
\qquad-\left.\frac{\partial \theta_{1}^{+}}{\partial y^{+}}\right|_{y^{+}=L_{y_{1}}^{+}}=\frac{h_{1}^{+}}{k_{1}^{+}} S t \cdot \operatorname{Pe}\left(\left.\theta_{1}^{+}\right|_{y^{+}=L_{y_{1}}^{+}}\right)\end{array}$ & $\begin{array}{l}\text { Back surface condition: } \\
\qquad\left.\frac{\partial \theta_{2}^{+}}{\partial y^{+}}\right|_{y^{+}=L_{y_{2}}^{+}}=S t \cdot \operatorname{Pe}\left(\left.\theta_{2}^{+}\right|_{y^{+}=L_{y_{2}}^{+}}\right)\end{array}$ \\
\hline & \multirow{2}{*}{\multicolumn{2}{|c|}{$\begin{array}{l}\text { Coupling condition (contact region): } \\
\qquad f_{d}^{+}\left(x^{+}\right)=\frac{1}{L_{y_{2}}^{+} P e}\left(\left.\frac{\partial \theta_{2}^{+}}{\partial y^{+}}\right|_{y^{+}=0}-\left.k_{1}^{+} \frac{\partial \theta_{1}^{+}}{\partial y^{+}}\right|_{y^{+}=0}\right) \\
\theta_{1}^{+}=\theta_{1}^{+} \text {con }\end{array}$}} \\
\hline & & \\
\hline $\begin{array}{l}\text { x-Boundary } \\
\text { Condition: }\end{array}$ & $\begin{aligned}\left.\frac{\partial \theta_{1}^{+}}{\partial x^{+}}\right|_{x^{+}=0} & =\frac{h_{1}^{+}}{k_{1}^{+}} S t \cdot \operatorname{Pe}\left(\left.\theta_{1}^{+}\right|_{x^{+}=0}\right) \\
-\left.\frac{\partial \theta_{1}^{+}}{\partial x^{+}}\right|_{x^{+}=L_{x_{1}}^{+}} & =\frac{h_{1}^{+}}{k_{1}^{+}} S t \cdot \operatorname{Pe}\left(\left.\theta_{1}^{+}\right|_{x^{+}=L_{x_{1}}^{+}}\right)\end{aligned}$ & $\begin{array}{l}\text { Wrapped condition: } \\
\qquad \begin{aligned} &\left.\theta_{2}^{+}\right|_{x^{+}=0}=\left.\theta_{2}^{+}\right|_{x}=L_{x_{2}}^{+} \\
&\left.\frac{\partial \theta_{2}^{+}}{\partial x^{+}}\right|_{x^{+}=0}=\left.\frac{\partial \theta_{2}^{+}}{\partial x^{+}}\right|_{x^{+}=L_{x_{2}}^{+}}\end{aligned}\end{array}$ \\
\hline $\begin{array}{l}\text { Initial } \\
\text { Condition: }\end{array}$ & $\left.\theta_{1}^{+}\right|_{t^{+}=0}=\theta_{1_{0}}^{+}\left(x^{+}, y^{+}\right)$ & $\left.\theta_{2}^{+}\right|_{t^{+}=0}=\theta_{2_{0}}^{+}\left(x^{+}, y^{+}\right)$ \\
\hline
\end{tabular}

In the same form as chapter 3 , these equations can be simplified down to the reduced cases. The non-dimensional form of the 2D, 1B set follows in Table 6.11. 
Table 6.11: Normalized 2D, 1B equations with dimensionless parameters.

\begin{tabular}{|c|c|c|}
\hline & & Body 2 \\
\hline $\begin{array}{l}\text { Energy } \\
\text { Equation: }\end{array}$ & & $\frac{\partial \theta_{2}^{+}}{\partial t^{+}}=\frac{1}{P e}\left(\frac{\partial^{2} \theta_{2}^{+}}{\partial x^{+^{2}}}+\frac{\partial^{2} \theta_{2}^{+}}{\partial y^{+^{2}}}\right)-\frac{\partial \theta_{2}^{+}}{\partial x^{+}}$ \\
\hline \multirow[t]{3}{*}{$\begin{array}{l}\text { y-Boundary } \\
\text { Condition: }\end{array}$} & Back surface condition: & $\left.\frac{\partial \theta_{2}^{+}}{\partial y^{+}}\right|_{y^{+}=L_{y_{2}}^{+}}=S t \cdot P e\left(\left.\theta_{2}^{+}\right|_{y^{+}=L_{y_{2}}^{+}}\right)$ \\
\hline & Contact condition: & $f_{d}^{+}\left(x^{+}\right)=\left.\frac{1}{L_{y_{2}}^{+} P e} \frac{\partial \theta_{2}^{+}}{\partial y^{+}}\right|_{y^{+}=0}$ \\
\hline & Non contact condition: & $-\left.\frac{\partial \theta_{2}^{+}}{\partial y^{+}}\right|_{y^{+}=0}=S t \cdot P e\left(\left.\theta_{2}^{+}\right|_{y^{+}=0}\right)$ \\
\hline $\begin{array}{l}\text { x-Boundary } \\
\text { Condition: }\end{array}$ & Wrapped condition: & $\begin{aligned}\left.\theta_{2}^{+}\right|_{x^{+}=0} & =\left.\theta_{2}^{+}\right|_{x^{+}=L_{x_{2}}^{+}} \\
\left.\frac{\partial \theta_{2}^{+}}{\partial x^{+}}\right|_{x^{+}=0} & =\left.\frac{\partial \theta_{2}^{+}}{\partial x^{+}}\right|_{x^{+}=L_{x_{2}}^{+}}\end{aligned}$ \\
\hline $\begin{array}{l}\text { Initial } \\
\text { Condition: }\end{array}$ & & $\left.\theta_{2}^{+}\right|_{t^{+}=0}=\theta_{2_{0}}^{+}\left(x^{+}, y^{+}\right)$ \\
\hline
\end{tabular}

The non-dimensional form of the 1D, 2B set follows in Table 6.12. 
Table 6.12: Normalized 1D, 2B equations with dimensionless parameters.

\begin{tabular}{|c|c|c|}
\hline & Body 1 & Body 2 \\
\hline $\begin{array}{l}\text { Energy } \\
\text { Equation: }\end{array}$ & $\begin{array}{c}\frac{\partial \theta_{1}^{+}}{\partial t^{+}}=\frac{\alpha_{1}^{+}}{P e} \frac{\partial^{2} \theta_{1}^{+}}{\partial x^{+2}}-\frac{h_{1}^{+} \alpha_{1}^{+}}{L_{y_{1}}^{+} k_{1}^{+}} S t \theta_{1}^{+} \\
+\frac{\alpha_{1}^{+}}{k_{1}^{+}} \frac{L_{y_{2}}^{+}}{L_{y_{1}}^{+}} f_{d}^{+}\left(x^{+}\right)\end{array}$ & $\begin{array}{c}\frac{\partial \theta_{2}^{+}}{\partial t^{+}}=\frac{1}{P e} \frac{\partial^{2} \theta_{2}^{+}}{\partial x^{+2}}-\frac{\partial \theta_{2}^{+}}{\partial x^{+}}+f_{d}^{+}\left(x^{+}\right)-\frac{S t}{L_{y_{2}}^{+}} \theta_{2}^{+} \\
\cdot\left(H\left(x^{+}-1\right)+1\right)\end{array}$ \\
\hline $\begin{array}{l}\mathrm{x} \text {-Boundary } \\
\text { Condition: }\end{array}$ & $\begin{array}{c}\left.\frac{\partial \theta_{1}^{+}}{\partial x^{+}}\right|_{x^{+}=0}=\frac{h_{1}^{+}}{k_{1}^{+}} S t \cdot \operatorname{Pe}\left(\left.\theta_{1}^{+}\right|_{x^{+}=0}\right) \\
-\left.\frac{\partial \theta_{1}^{+}}{\partial x^{+}}\right|_{x^{+}=L_{x_{1}}^{+}}=\frac{h_{1}^{+}}{k_{1}^{+}} S t \cdot \operatorname{Pe}\left(\left.\theta_{1}^{+}\right|_{x^{+}=L_{x_{1}}^{+}}\right)\end{array}$ & $\begin{array}{l}\text { Wrapped Condition: } \\
\qquad \begin{aligned} &\left.\theta_{2}^{+}\right|_{x^{+}=0}=\left.\theta_{2}^{+}\right|_{x^{+}=L_{x_{2}}^{+}} \\
&\left.\frac{\partial \theta_{2}^{+}}{\partial x^{+}}\right|_{x^{+}=0}=\left.\frac{\partial \theta_{2}^{+}}{\partial x^{+}}\right|_{x^{+}=L_{x_{2}}^{+}}\end{aligned}\end{array}$ \\
\hline $\begin{array}{l}\text { Initial } \\
\text { Condition: }\end{array}$ & $\left.\theta_{1}^{+}\right|_{t^{+}=0}=\theta_{1_{0}}^{+}\left(x^{+}\right)$ & $\left.\theta_{2}^{+}\right|_{t^{+}=0}=\theta_{2_{0}}^{+}\left(x^{+}\right)$ \\
\hline
\end{tabular}

And finally, for the 1D, 1B set, the non-dimensional forms are as follows in Table 6.13.

Table 6.13: Normalized 1D, 1B equations with dimensionless parameters.

\begin{tabular}{|c|c|}
\hline & Body 2 \\
\hline Energy Equation: & $\frac{\partial \theta_{2}^{+}}{\partial t^{+}}=\frac{1}{P e} \frac{\partial^{2} \theta_{2}^{+}}{\partial x^{+^{2}}}-\frac{\partial \theta_{2}^{+}}{\partial x^{+}}+f_{d}^{+}\left(x^{+}\right)-\frac{S t}{L_{y_{2}}^{+}} \theta_{2}^{+} \cdot\left(H\left(x^{+}-1\right)+1\right)$ \\
\hline $\begin{array}{l}\text { x-Boundary } \\
\text { Condition: }\end{array}$ & $\begin{array}{l}\text { Irapped condition: } \\
\qquad \begin{aligned}\left.\theta_{2}^{+}\right|_{x^{+}=0} & =\left.\theta_{2}^{+}\right|_{x^{+}=L_{x}^{+}} \\
\left.\frac{\partial \theta_{2}^{+}}{\partial x^{+}}\right|_{x^{+}=0} & =\left.\frac{\partial \theta_{2}^{+}}{\partial x^{+}}\right|_{x^{+}=L_{x_{2}}^{+}}\end{aligned}\end{array}$ \\
\hline Initial Condition: & $\left.\theta_{2}^{+}\right|_{t^{+}=0}=\theta_{2_{0}}^{+}\left(x^{+}\right)$ \\
\hline
\end{tabular}




\subsection{High Peclet Simplification}

The need for the diffusion process, particularly in the one dimensional models, can vary. In many circumstances, the heat transfer along the ' $x$ ' direction is highly dominated by the advective transfer. This relation can be seen by taking the ratios of the processes:

$$
\frac{\left\|\left.\frac{\partial T}{\partial t}\right|_{a d v}\right\|}{\left\|\left.\frac{\partial T}{\partial t}\right|_{d i f f}\right\|}=\frac{\left\|V \frac{\partial T}{\partial x}\right\|}{\left\|\alpha \frac{\partial^{2} T}{\partial x^{2}}\right\|}
$$

If one views the heat transfer from the perspective of the bulk behavior of the moving solid, one gains the picture of having three distinct regions near the contact zone. This is represented in Figure 6.1.

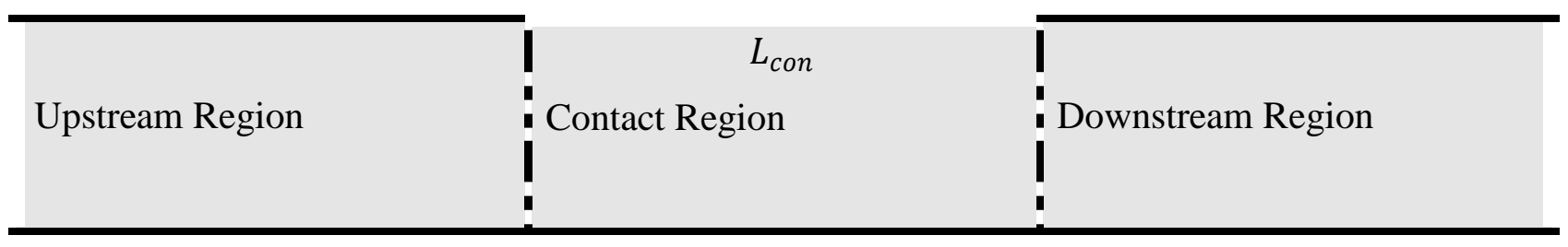

Figure 6.1: Bulk visualization for determining physical length scale.

The convenient physical length scale for determining the bulk heat transfer behaviors is $L_{c o n}$. Thus, approximating the above ratio with finite difference based on that scale gives the Peclet Number:

$$
\frac{\left\|V \frac{\partial T}{\partial x}\right\|}{\left\|\alpha \frac{\partial^{2} T}{\partial x^{2}}\right\|} \cong \frac{V \frac{\Delta T}{L_{c o n}}}{\alpha \frac{\Delta T}{L_{c o n}^{2}}}=\frac{V \cdot L_{c o n}}{\alpha}=P e
$$

Thus, if the Peclet Number is greater than about 10 or 100, the diffusion heat transfer is overwhelmed by the advective transfer. For instance, if one takes a braking system for a car, with 16" wheels, a 10" steel brake rotor, with a 2" long pad, and is traveling at $75 \mathrm{mph}$, the Peclet Number is approximately 60000. This result means that the advective transfer is approximately 60000 times greater than the diffusive behavior, thus overwhelming the diffusive transfer.

In the event where the Peclet Number does not support ignoring the ' $x$ ' diffusion, or when taking a two dimensional model where gradients in ' $y$ ' cannot be neglected, then one must solve Equation 4.38 numerically. 


\subsection{One Body Problems}

Presented here are the one body problem results. These results present many issues involved in estimating the discretized contact distribution (as seen in Figure 5.5) using measurements in the moving body. These results, in reality, most resemble either those of a low conductivity stationary body, or a moving body with large velocity since the moving body is assumed to get all of the frictional heating.

\subsubsection{D, 1B Cases}

The 1D, 1B construct is a very useful starting point. It demonstrates many of the difficulties involved in measurement, as well as many of the basic trends, without being excessively computationally intensive. This construct grants a quick, but coarse view of the system. The important assumption in the one dimensional case is that the material is thin enough to approximate the temperature as being the same across the ' $y$ ' direction. The non-dimensional formulation is presented above in Table 6.13. For $P e \gg 1$, the required dimensionless parameters reduce to $L_{x}^{+}, \frac{S t}{L_{y}^{+}}$, and $f_{d}^{+}$.

\section{Numerical Resolution Case}

This first case looks at the direct model, to determine how fine of a spatial resolution is necessary to achieve good enough convergence. Most importantly, the results must all exhibit the same trends, and be roughly the same value. Because the application is the determination of trends, an exact convergence is not required. Presented below in Table 6.14 are the physical system parameters used to determine the temperature distribution. A flat or uniform contact area covering $10 \%$ of the moving body is used. The direct model solutions with 100 and 200 nodes are presented here. This results in 10 and 20 discrete contacts, respectively.

Table 6.14: Physical parameters for the numerical resolution case.

\begin{tabular}{|c|c|}
\hline \multicolumn{2}{|c|}{ Physical Parameters } \\
\hline$L_{x}^{+}$ & 10 \\
\hline$\frac{S t}{L_{y}^{+}}$ & 0.1 \\
\hline$f_{d}^{+}\left(x^{+}\right)$ & $\begin{cases}1 & x^{+} \leq 1 \\
0 & x^{+}>1 \\
\hline\end{cases}$
\end{tabular}


With the use of these parameters, the temperature solution can be determined as seen below in Figure 6.2. The first plot in the figure shows the solution with 100 nodes. The second plot shows the solution with 200 nodes. These plots qualitatively demonstrate similar behaviors.

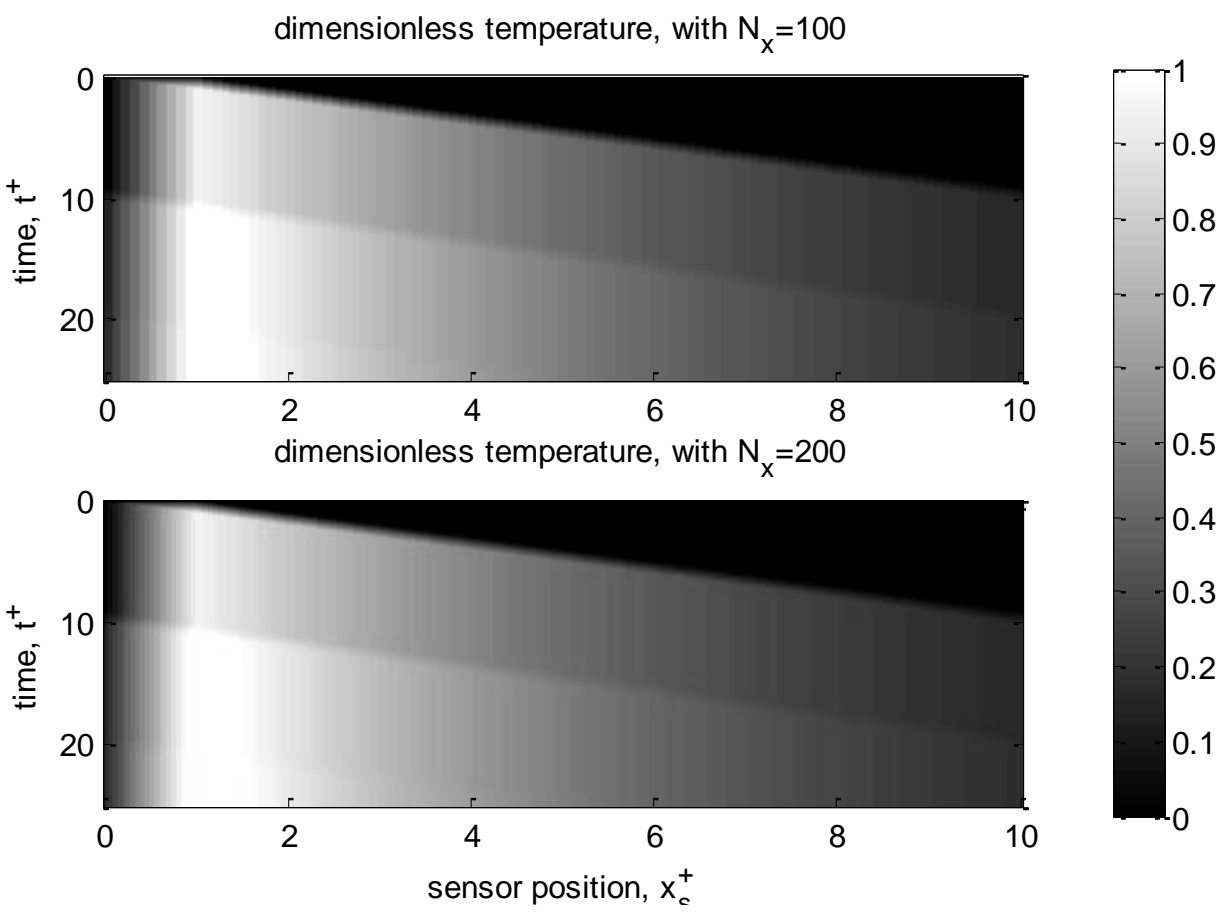

Figure 6.2: Temperature fields for 100 and 200 node simulations.

Of particular interest is that the 'front' of the propagating heat is visible. As a result of the wrapped boundary condition, the temperature 'steps' up dramatically every time the material has had a chance to cycle through every location. That means, every $t^{+}=10$, there is a step up, until the solution achieves a steady solution as a result of the convective loss. With regard to the numerical convergence of the solution, there is no immediately visible difference in the solution between the two graphs above. Thus, for the purposes of this study, 100 nodes for the $1 \mathrm{D}$ case is sufficiently converged.

\section{Example Case}

This first estimation case presented here is to provide a sample result for a single run of the parameter estimation technique. The system presented below has physical and measurement parameters listed in Table 6.15. This uses the same 100 node discretization scheme, which was 
shown to provide sufficient accuracy, from the above numerical resolution case. This scheme results in 10 discrete values of the contact distribution.

Table 6.15: Parameter values for sample case.

\begin{tabular}{|c|c|}
\hline \multicolumn{2}{|c|}{ Physical Parameters } \\
\hline$L_{x}^{+}$ & 10 \\
\hline$\frac{S t}{L_{y}^{+}}$ & 0.1 \\
\hline$f_{d}^{+}\left(x^{+}\right)$ & $\begin{cases}1 & x^{+} \leq 1 \\
0 & x^{+}>1\end{cases}$ \\
\hline \multicolumn{2}{|c|}{ Measurement Parameters } \\
\hline$N_{s}$ & 1 \\
\hline$x_{s}^{+}$ & 1.5 \\
\hline $\pm e r r^{+}$(simulated) & \pm 0.02 \\
\hline
\end{tabular}

Upon running the case presented above, data was simulated using the above provided error, in conjunction with MATLAB's pseudo-random number generator, which is defined from zero to one. Thus, this simulated data was generated by the following method:

$$
Y_{m}^{+}=T_{\text {exact }}^{+}+\text {err }^{+} \cdot 2\left(\operatorname{rand}\left(\operatorname{length}\left(t^{+}\right), 1\right)-.5\right)
$$

The dimensionless temperature rise over time for the selected measurement location is presented below in Figure 6.3. This shows the variations seen in the data as a result of simulated measurement error.

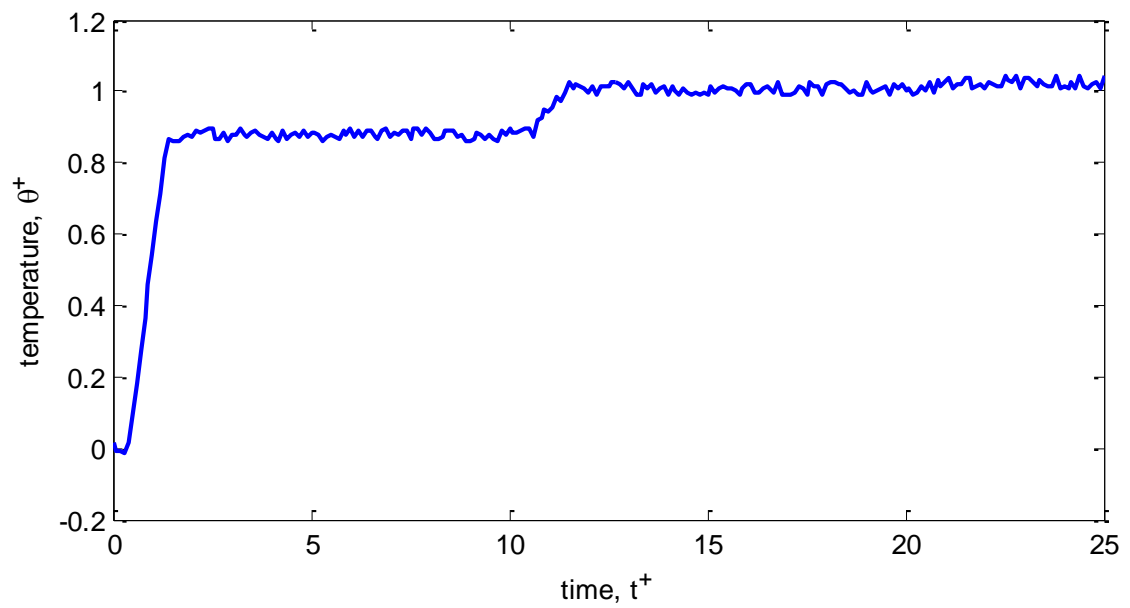

Figure 6.3: Sample dimensionless temperature data taken at a normalized location of $x_{s}^{+}=1.5$. 
There are several interesting characteristics in this graph. First, there is the expected lag of $\Delta t^{+}=0.5$ in the data before any action happens, as the heated material still must transport forward a distance of $\Delta x^{+}=0.5$. Second, a temperature rise is observed upon every complete cycle. This step happens every dimensionless time of $t^{+}=10$ since $L_{x}^{+}=10$. This data is then used in the parameter estimation software discussed above in chapter 5. The software then provides the answer that causes the model to most closely mimic the data. Since $\Delta x^{+}=0.1$, there are ten parameters to be estimated. The contact distribution estimated by the parameter estimation routine is presented in Figure 6.4.

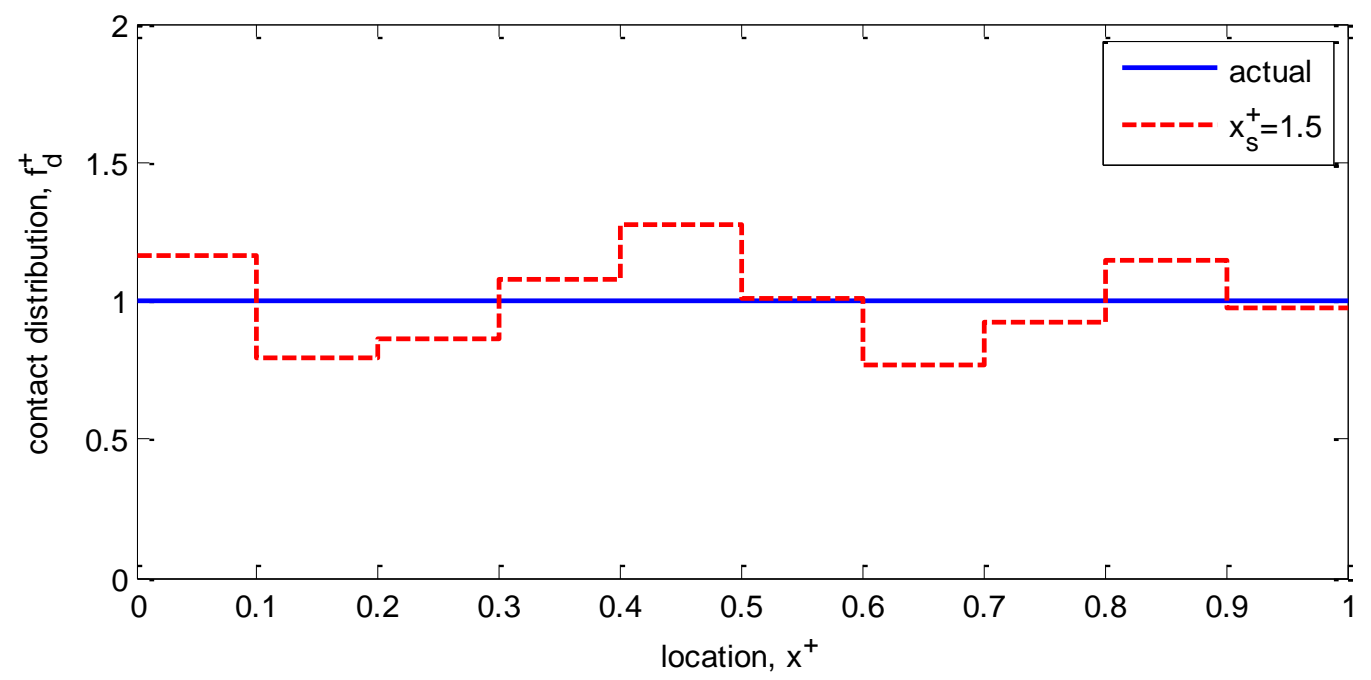

Figure 6.4: Dimensionless contact distribution comparison.

The actual distribution is uniform over the nominal contact. The parameter estimation method is able to simulate this distribution reasonably well. This demonstrates that the quality of the estimation is good enough to provide a rough estimate of the scale and distribution of the contact.

\section{Contact Distribution Study}

This next case is presented to demonstrate the behavior of the estimation method for a variety of contact distributions. Four distributions are utilized. These consist of a uniform distribution, a Hertzian distribution (the hump-like distribution from Figure 3.2), a distribution with three uniformly distributed contacts, and a randomly assigned distribution. Presented below in Table 6.16 are the physical parameters used to generate the data. 
Table 6.16: Parameter values for contact distribution study.

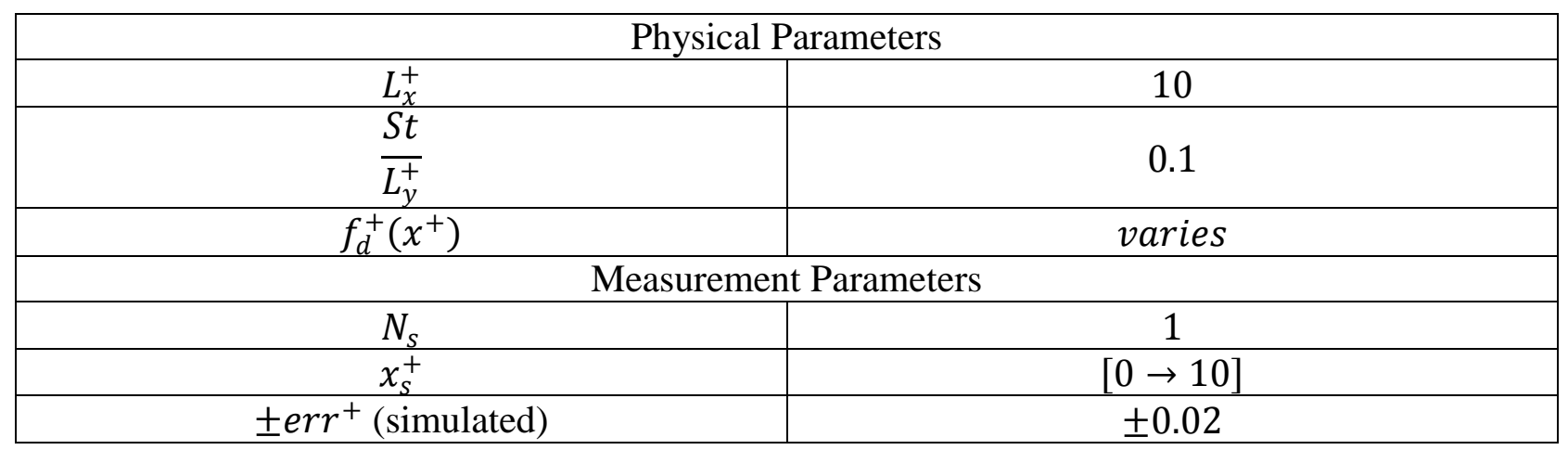

Upon running the above cases, raw data was simulated in the same way as the example. The dimensionless temperature rise was simulated in all nodes. A sample of the simulated data is provided below in Figure 6.5. Again, it should be noted that the characteristics mentioned in the example case still applies here.
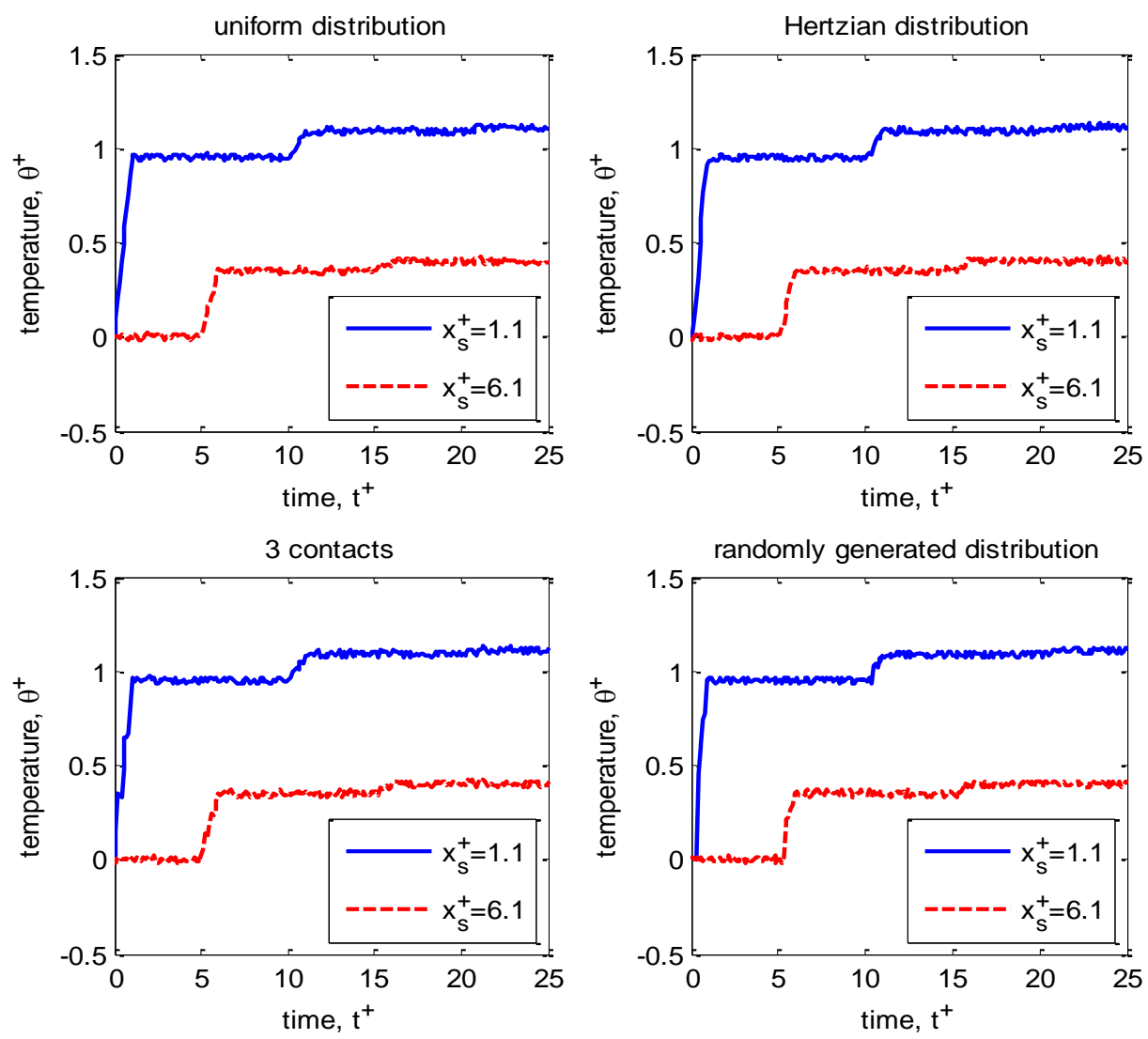

Figure 6.5: Dimensionless data simulated at 1.1 and 6.1 contact lengths. 
These data were used to estimate the contact distributions as seen below in Figure 6.6.
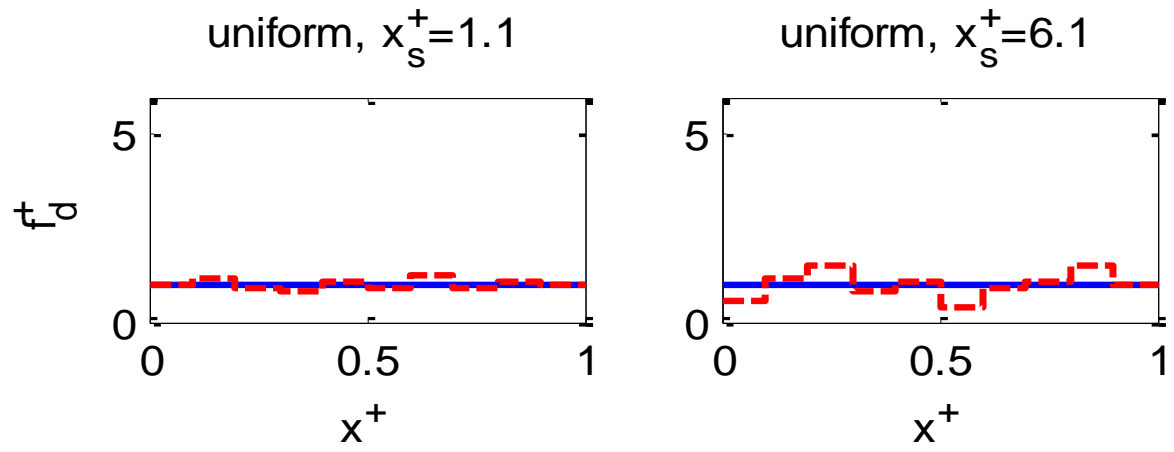

Hertzian, $x_{s}^{+}=1.1$

Hertzian, $x_{s}^{+}=6.1$
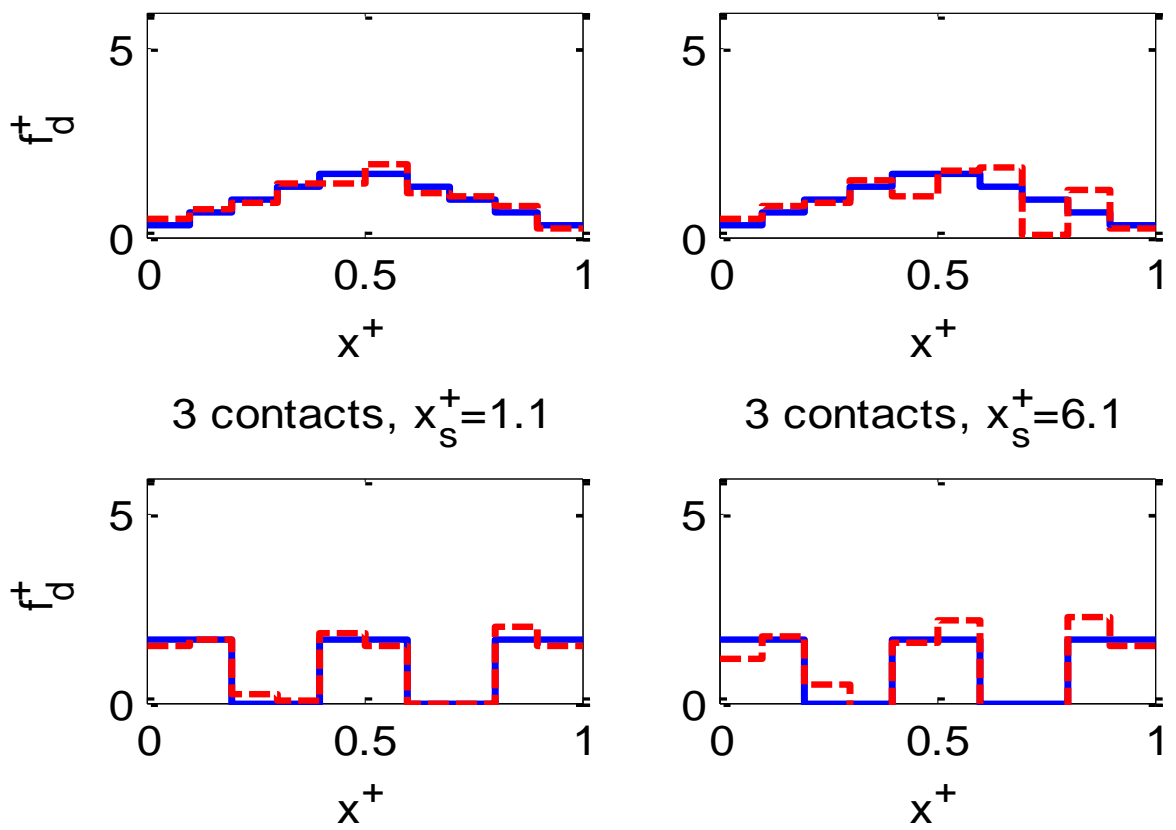

$$
\text { random, } x_{s}^{+}=1.1
$$

random, $x_{s}^{+}=6.1$
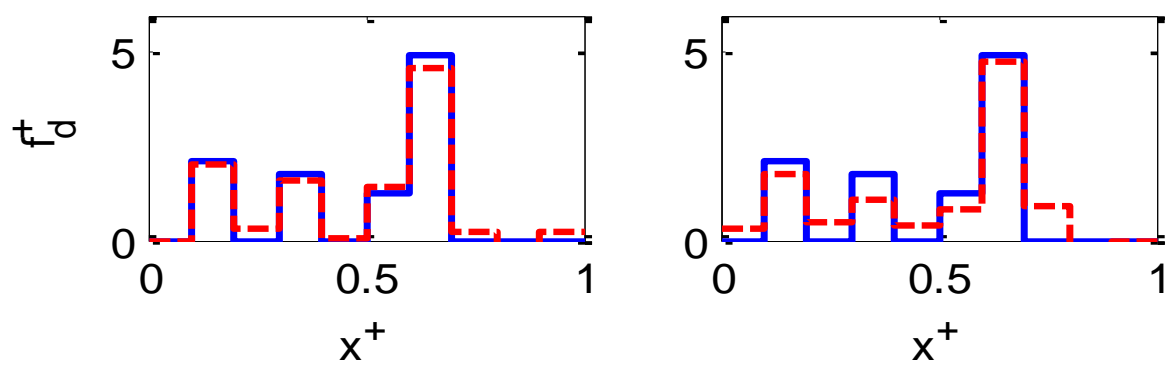

Figure 6.6: Estimated dimensionless contact distributions for various input distributions. The actual distribution is the solid line, and the estimated distribution is the dashed line. 
It can be noted that, for $x_{s}^{+}=6.1$, the estimation is of a noticeably lower quality. This makes sense because it is farther away than the $x_{s}^{+}=1.1$ case, which estimated the distribution quite well. The topography of the estimation very nearly matches that of what the input distribution actually was. This shows that the estimation method works well, not at just determining the average contact, as the flat distribution would indicate, but also a variety of others. Of particular interest is that the computer could randomly generate a distribution like in the fourth case, and the estimation technique could determine that distribution. This indicates that the solution does not bias towards a given result.

As part of the estimation routine, the sensitivity of the temperature to the parameters is computed. Thus, for each measurement, since there are ten parameters, there are ten sensitivity time traces. These can be used to demonstrate numerically why the quality of the estimation by the closer node is better than that of the farther node. The sensitivities are calculated by:

$$
X_{n}^{+}=\left.\frac{\partial \theta^{+}}{\partial \beta_{n}}\right|_{x^{+}=x_{s}^{+}}
$$

The sensitivity traces for a sample of the parameters is shown below in Figure 6.7. The sensitivity is significantly higher for the closer node than the further node. Also, since the model is linear in the parameters, the sensitivities do not change with the contact distribution. This graph also only has the sensitivity traces with respect to the first, fourth, seventh, and tenth parameters.
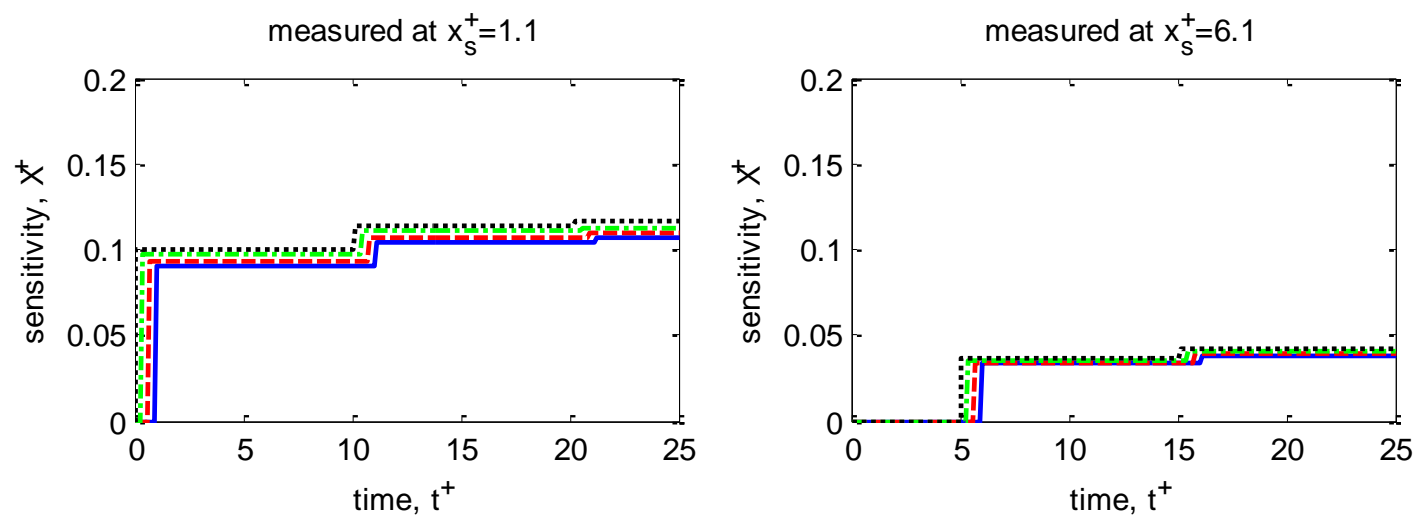

Figure 6.7: Selected sensitivity coefficients at measurement locations of 1.1 and 6.1. The selected parameters were $\beta_{1}, \beta_{4}, \beta_{7}$, and $\beta_{10}$. These corresponded to the value of the contact distribution at $\boldsymbol{x}^{+}=[0.1,0.4,0.7,1.0]$ 
The above graphs are useful, but because there can be many parameters, it can be very difficult to look at the sensitivities by themselves. However, by taking the RMS difference of the contact distributions for each of the estimations, one can arrive at a convenient means of determining the preferred measurement location. This RMS value is achieved by:

$$
\Delta f_{d_{R M S}}^{+}=\sqrt{\frac{1}{N} \sum_{n=1}^{N}\left(\beta_{n}-f_{d_{n}}^{+}\right)^{2}}
$$

This method essentially seeks the minimum RMS difference as a function of measurement location. Thus, by characterizing the quality of the estimation against the input distribution with a single value, then the data can be plotted in a convenient form. This is shown below in Figure 6.8 .

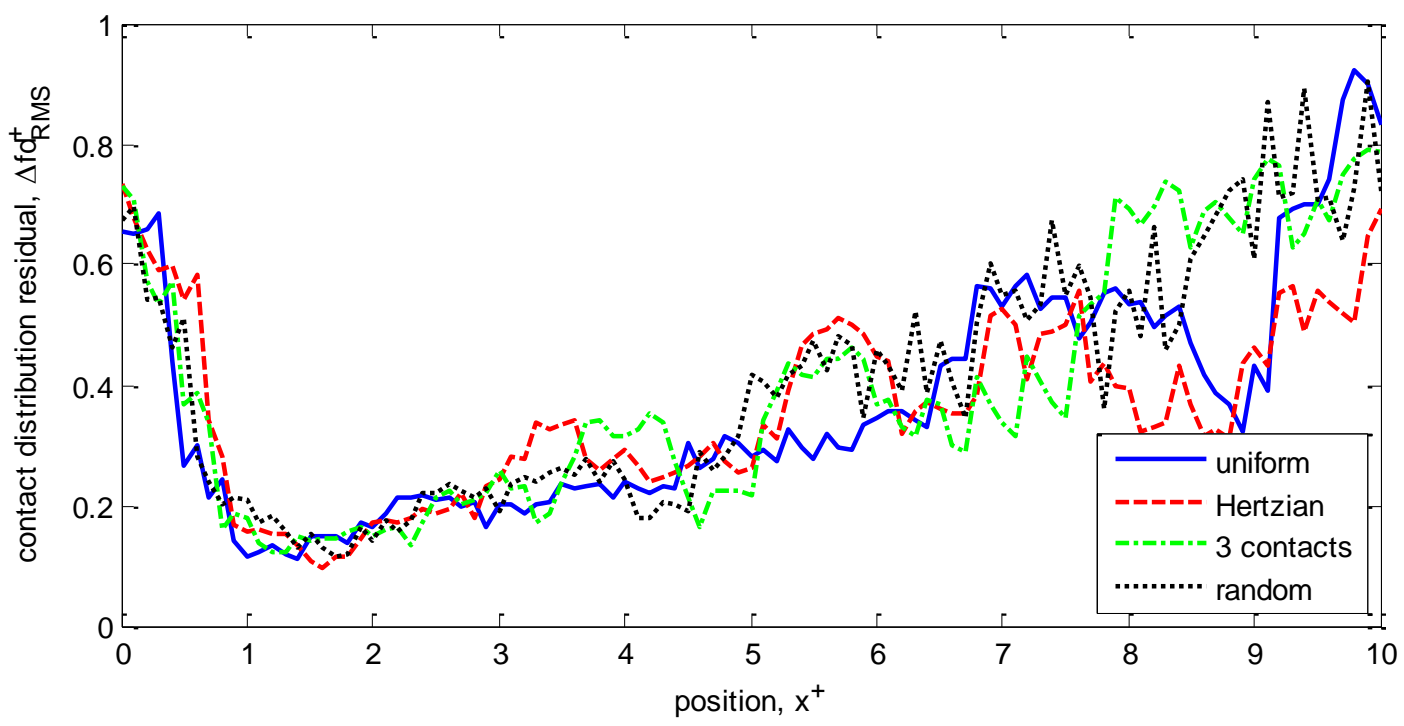

Figure 6.8: Contact distribution RMS residuals, with varying contact distributions.

This image shows that, regardless of the distribution being measured, the preferred measurement location is just downstream of the contact, with $1<x_{s}^{+}<2$. However, that information is available at some points further downstream. Care must be taken, however, that one is not in the insensitive region. In the far away region $x_{s}^{+}>7.5$, the data appears insensitive to the contact distribution, and as such, any distribution (within broad limitations) will result in a converged answer. That is useless. Thus, these results demonstrate two conclusions: that the preferred placement of the measurement does not change between distributions, and that the 
estimation system can return a reasonably accurate result without any form of a priori knowledge about the distribution, beyond the nominal contact zone.

\section{Effect of Error}

The next case is presented to show the effect of the simulated error. As a means to avoid observing 'wrap around' effects of the bulk transport, the $\frac{S t}{L_{y}^{+}}$term has been increased to ensure the data achieves a roughly steady state during the first cycle. The cases involved will study the effects of error on the $\Delta f_{d_{R M S}}^{+}$plot. The physical and measurement parameters as they were used for this study are below in Table 6.17.

Table 6.17: Parameter values used in the varied error case.

\begin{tabular}{|c|c|}
\hline \multicolumn{2}{|c|}{ Physical Parameters } \\
\hline$L_{x}^{+}$ & 10 \\
\hline$\frac{S t}{L_{y}^{+}}$ & 1 \\
\hline$f_{d}^{+}\left(x^{+}\right)$ & $\begin{cases}1 & x^{+} \leq 1 \\
0 & x^{+}>1\end{cases}$ \\
\hline \multicolumn{2}{|c|}{ Measurement Parameters } \\
\hline$N_{S}$ & 1 \\
\hline$x_{S}^{+}$ & {$[0 \rightarrow 10]$} \\
\hline $\pm e r r^{+}($simulated $)$ & $\pm[0,0.01,0.02]$ \\
\hline
\end{tabular}

The error was simulated once and then applied to each 'actual condition' node as required. These error traces are presented below in Figure 6.9. 


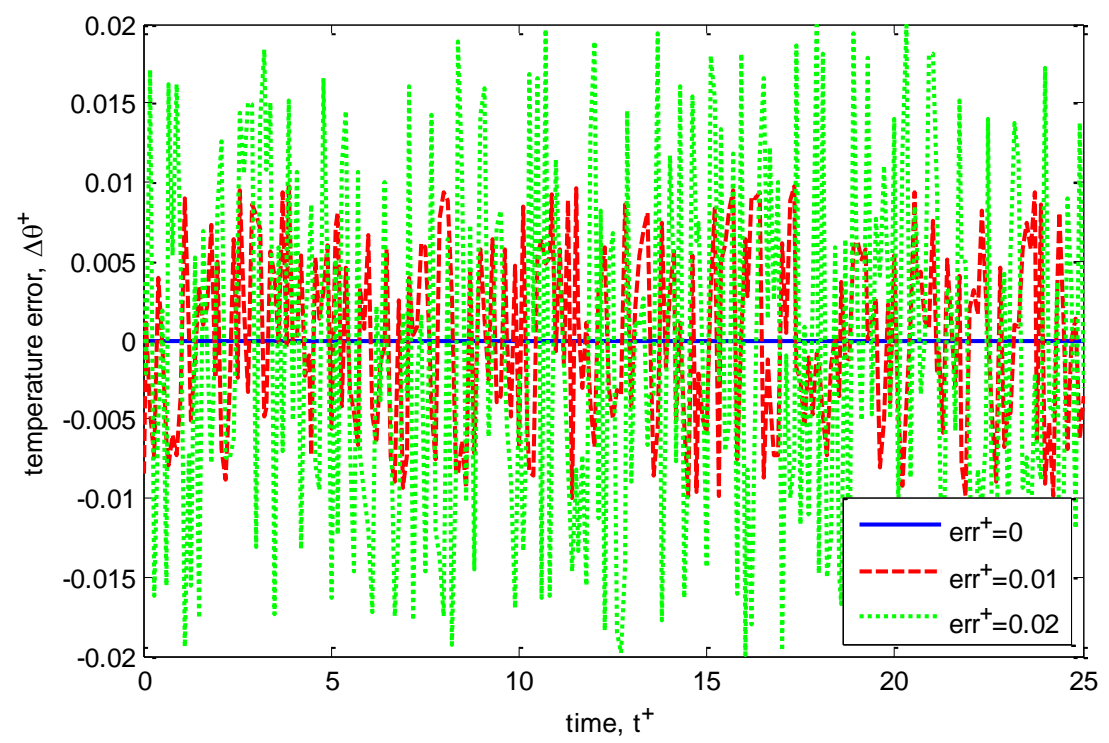

Figure 6.9: Simulated error traces.

As was done above in Figure 6.8, the contact distribution RMS residual plot was generated for each error trace. This is presented below in Figure 6.10. This results in roughly what one would expect, where the zero-error case has a rather smooth curve, and has the lowest valued minimum. The other traces are rougher, but follow the same basic shape, with larger residuals.

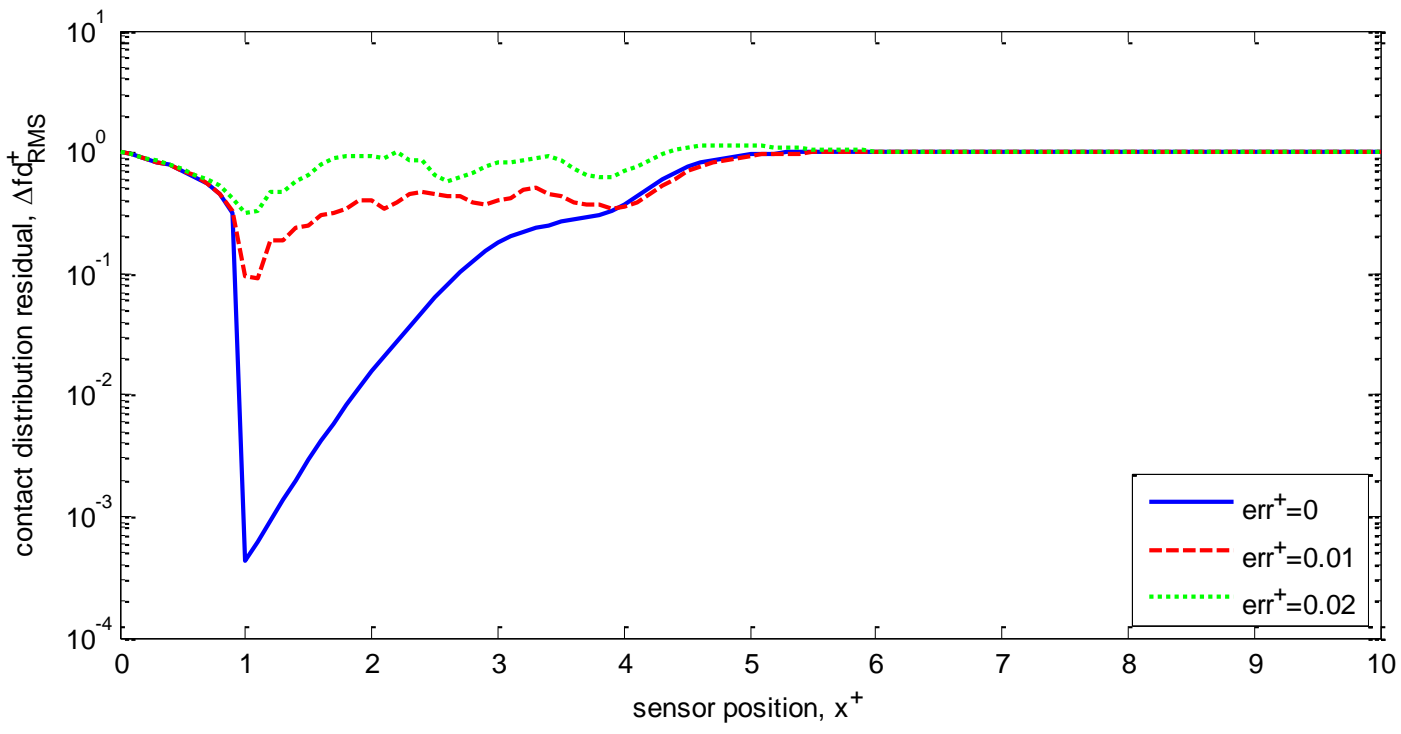

Figure 6.10: Contact distribution RMS residual for varied error case. 
Of particular note from this graph is the behavior of the roll-off from $x_{s}^{+}=0$ to 1 . The leveling off of information shows that the method, when there is no demonstrable sensitivity to a parameter, leaves that parameter at its initial seed value. The initial seed values used throughout this paper were zero (indicative of zero contact). Thus, because the model neglects axial conduction, the first node is only sensitive to the first contact parameter, and highly insensitive to all the others. Thus it predicts a distribution of $[1,0,0,0,0,0,0,0,0,0]$. The RMS difference between the two is $\sqrt{0.9}$. The next node has sensitivity to the first two nodes, and thus has an RMS difference of $\sqrt{0.8}$. This leads to the important observation that measurements taken where the RMS residual is unity indicates zero sensitivity to the estimation parameters, and thus that measurement location gives no indication of the contact area. Further, this roll-off behavior seems to be preserved throughout the selected error parameters.

\section{Effect of the Length of the Solid}

This next case is presented to show the effect of the length of the rotating body. In particular, this study involves a fixed number of parameters constant and constant nodal spacing, while allowing the overall length to change. Further, to observe the effect of any wrap around effects in the simulation, the Stanton parameter was varied. The physical and measurement parameters as they were used for this study are below in Table 6.18.

Table 6.18: Parameter values used in the total length study.

\begin{tabular}{|c|c|}
\hline \multicolumn{2}{|c|}{ Physical Parameters } \\
\hline$L_{x}^{+}$ & {$[2,5,10]$} \\
\hline$\frac{S t}{L_{v}^{+}}$ & {$[5,1,0.5]$} \\
\hline$f_{d}^{+}\left(x^{+}\right)$ & $\begin{cases}1 & x^{+} \leq 1 \\
0 & x^{+}>1\end{cases}$ \\
\hline \multicolumn{2}{|c|}{ Measurement Parameters } \\
\hline$N_{s}$ & 1 \\
\hline$x_{s}^{+}$ & {$\left[0 \rightarrow L_{x}^{+}\right]$} \\
\hline$\pm \operatorname{err}^{+}$(simulated $)$ & \pm 0.02 \\
\hline
\end{tabular}

The RMS contact distribution residual $\left(\Delta f_{d_{R M S}}^{+}\right)$traces of this case are shown below in Figure 6.11. For $\frac{S t}{L_{y}^{+}}=5$, the traces all fall directly on top of each other. This is what one would 
anticipate as the result because, as stated above, the Stanton parameter was selected to prevent the appearance of wrap around effects. Thus, the material must be roughly ambient temperature by $x^{+}=2$. Otherwise, if the data is allowed to wrap around, the quality of the data in the shorter material in the contact region is improved over the others because the information from the previous iteration is preserved. As $\frac{S t}{L_{y}^{+}}$is allowed to decrease for a given $L_{x}^{+}$, the residuals get smaller, and the effective measurement range increases.

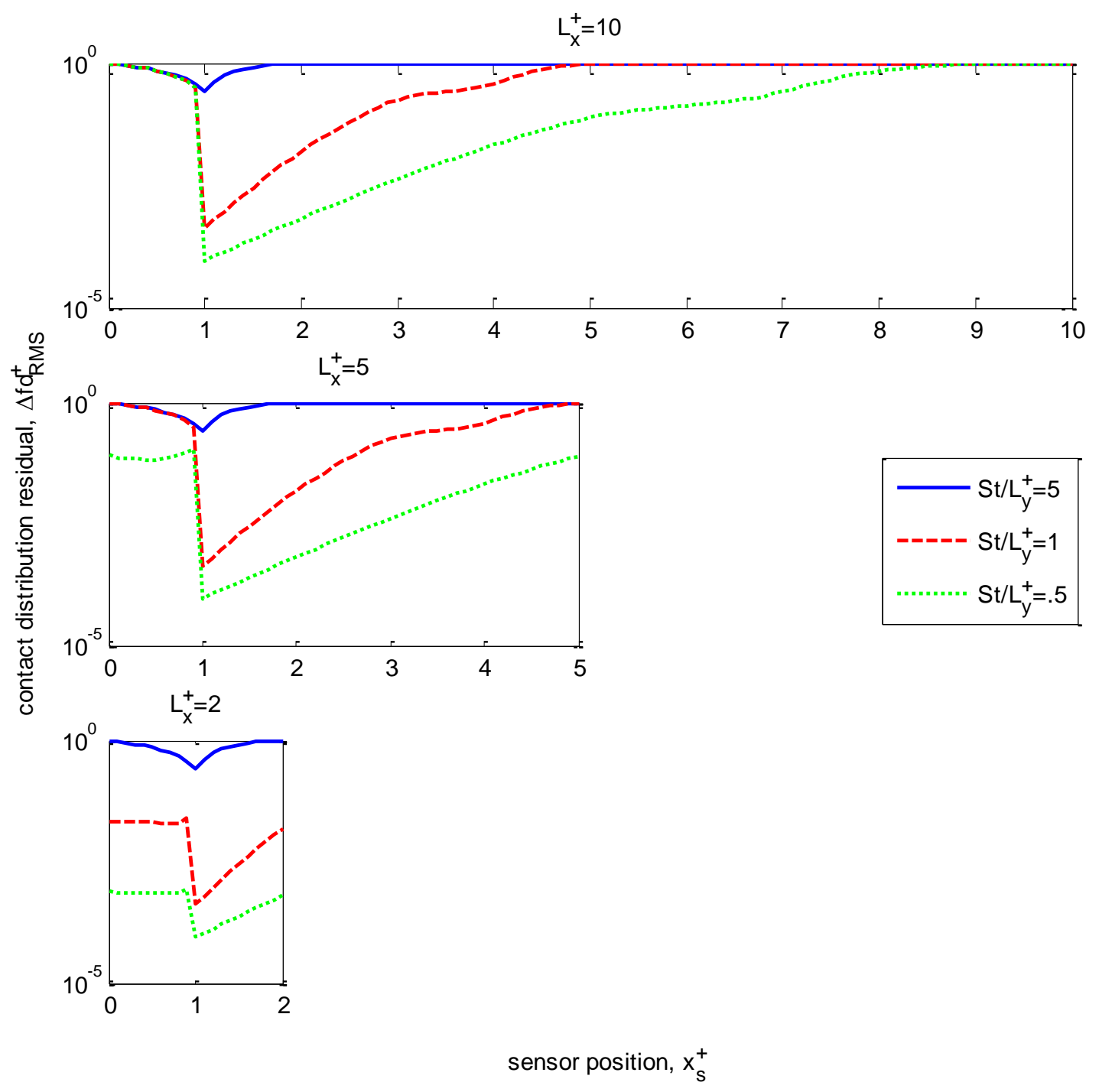

Figure 6.11: Contact distribution residual for the total lengths of $L_{x}^{+}=10,5,2$.

One of the critical observations from this graph is that the preferred measurement location seems to always situate itself on $x_{s}^{+}=1$. This makes intuitive sense as that is always 
the immediate downstream location that has the best access to information about the action from the whole contact.

\section{Effect of the Nominal Contact Zone}

This next case is presented to show the effect of the length of the nominal contact area. In particular, this study involves varying the number of parameters while maintaining the same nodal spacing and overall length $L_{x}$. The physical and measurement parameters as they were used for this study are below in Table 6.19.

Table 6.19: Parameter values used in the contact length study.

\begin{tabular}{|c|c|}
\hline \multicolumn{2}{|c|}{ Physical Parameters } \\
\hline$L_{x}^{+}$ & {$[2,5,10]$} \\
\hline$\frac{S t}{L_{v}^{+}}$ & 0.1 \\
\hline$f_{d}^{+}\left(x^{+}\right)$ & $\begin{cases}1 & x^{+} \leq 1 \\
0 & x^{+}>1\end{cases}$ \\
\hline \multicolumn{2}{|c|}{ Measurement Parameters } \\
\hline$N_{s}$ & 1 \\
\hline$x_{s}^{+}$ & {$\left[0 \rightarrow L_{x}^{+}\right]$} \\
\hline $\pm e r r^{+}$(simulated $)$ & \pm 0.02 \\
\hline
\end{tabular}

The critical results of this study are the behavior of the RMS contact residual trace as a function of $L_{x}^{+}$. Presented below in Figure 6.12 are the RMS residual traces for the above prescribed values of $L_{x}^{+}$. As $L_{x}^{+}$increases, since the total number of nodes was maintained to be the same, the number of parameters to be estimated is decreased. Thus, when $L_{x}^{+}=2$, the nominal contact consumes half the available surface. This means that sensors located immediately downstream of the contact are still half the total length away from the first node of potential contact, thus reducing its sensitivity to the value of the contact there. 


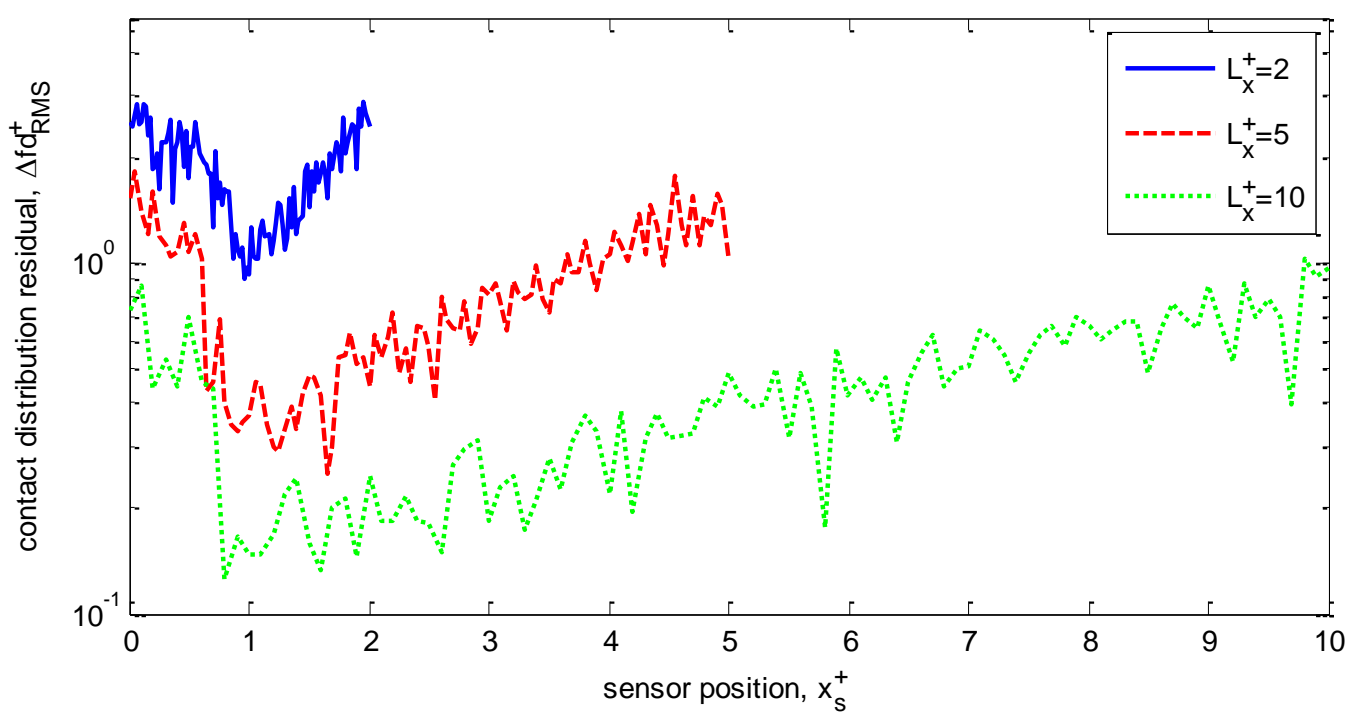

Figure 6.12: Contact distribution RMS residual for various dimensionless lengths.

Further, as the number of parameters increases, it is obvious that the method itself becomes unstable. A single data trace of 250 points was insufficient to gain any accuracy about the topography of the contact when the number of parameters being estimated was $50\left(L_{x}^{+}=2\right)$. This is well shown below in Figure 6.13. This is the contact distribution as estimated by $x_{s}^{+}=1$.

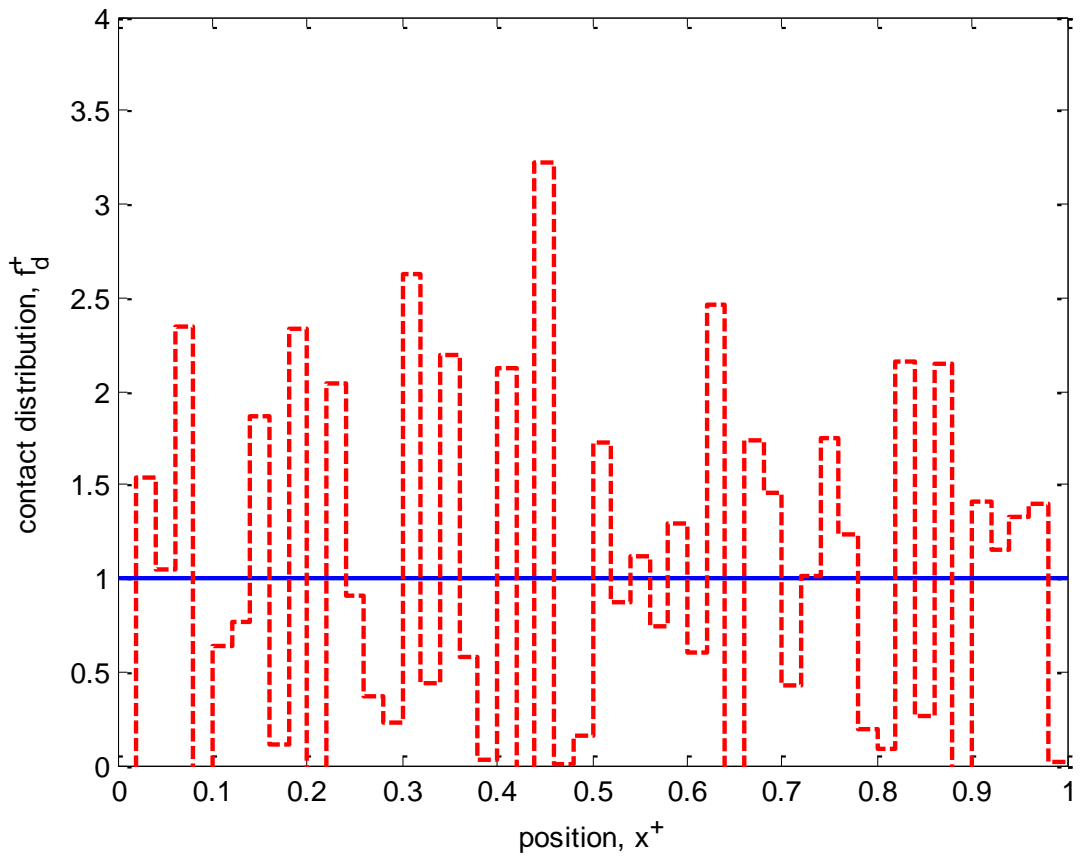

Figure 6.13: Sample contact distribution from the 50 parameter case. The 50 parameter case utilized a value of $L_{x}^{+}=2$. 
It should be noted that, while the average of the estimated distribution is indeed very close to 1 , it maintains no sense of the actual topography of the input function.

\section{Number of Sensors Study}

This final case study for the 1D, 1B model discusses the preferred positioning of up to three sensors worth of data collection. This is important because, if an experimenter has access to multiple measurement devices (as is frequently the case), understanding the interaction of the emplacements is essential to getting the best possible results from the measurement construct. The physical and measurement parameters as they were used for this study are below in Table 6.20 .

Table 6.20: Parameter values for the multi-sensor system.

\begin{tabular}{|c|c|}
\hline \multicolumn{2}{|c|}{ Physical Parameters } \\
\hline$L_{x}^{+}$ & {$[2,5,10]$} \\
\hline$\frac{S t}{L_{v}^{+}}$ & 1 \\
\hline$f_{d}^{+}\left(x^{+}\right)$ & $\begin{cases}1 & x^{+} \leq 1 \\
0 & x^{+}>1\end{cases}$ \\
\hline \multicolumn{2}{|c|}{ Measurement Parameters } \\
\hline$N_{S}$ & {$[1,2,3]$} \\
\hline$x_{s}^{+}$ & {$[0 \rightarrow 10]$} \\
\hline $\pm e r r^{+}($simulated $)$ & \pm 0.0 \\
\hline
\end{tabular}

Firstly, and most obviously, it is important to know where to measure with a single sensor. Thus, recalling from Figure 6.10, the contact distribution RMS residual for a single sensor shows the intuitive result that one would place the sensor immediately downstream of the contact.

In order to present the interaction of two sensors, the RMS residual data was generated for the entire nodal matrix. This sweep enables a density plot to be generated of the data as it varies in both variables. This can be seen below in Figure 6.14, where the darker the region, the smaller the value of the residual. Most of the graph is showing white, or nearly so. This is indicative of regions of essentially zero sensitivity, where one would not want to take data, if one 
was interested in the contact distribution. It should be noted that the same basic characteristics of the above graph are followed, in that the region where good measurements can be achieved is roughly between $x_{s}^{+}=1$ and 4 . It is notable that the minimum of the data shows that, at least computationally, the preferred measurement locations are immediately under the nodes at the end of the contact. This result is not anticipated, but makes sense as long as one recalls that the data was generated using zero simulated error. Thus, maintaining maximum sensitivity to as many nodes as possible is the best possible way to estimate the contact distribution.
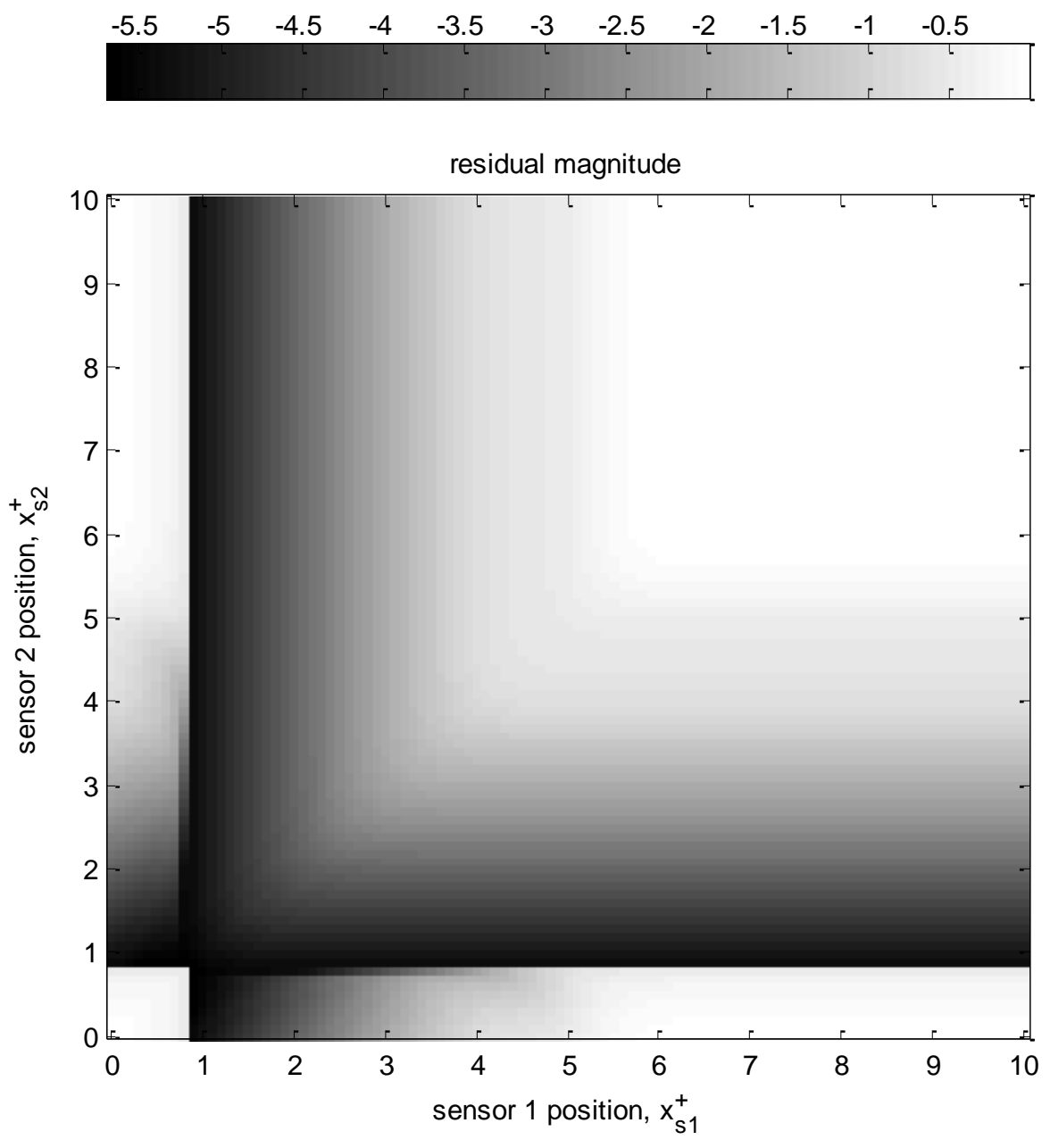

Figure 6.14: Contact distribution RMS residual density plot.

In order to present data on three sensors, one must generate a series of density plots. This is done by taking sections of the data with one sensor fixed. This result grants a density plot of the data as the other two sensors vary. Luckily, having done both the one sensor and two sensor 
cases, we know that a reasonable set of locations to take the sections is around the downstream nodes of contact. Thus, the sections will be taken at $x_{s_{3}}^{+}=[0.8,0.9,1.0,1.1]$. Further, as a result of the above data, the amount of data necessary to be generated to come to acceptable conclusions has been reduced, and thus data was only generated between $x_{s}^{+}=0$ and 2.5. These data are presented below in Figure 6.15.
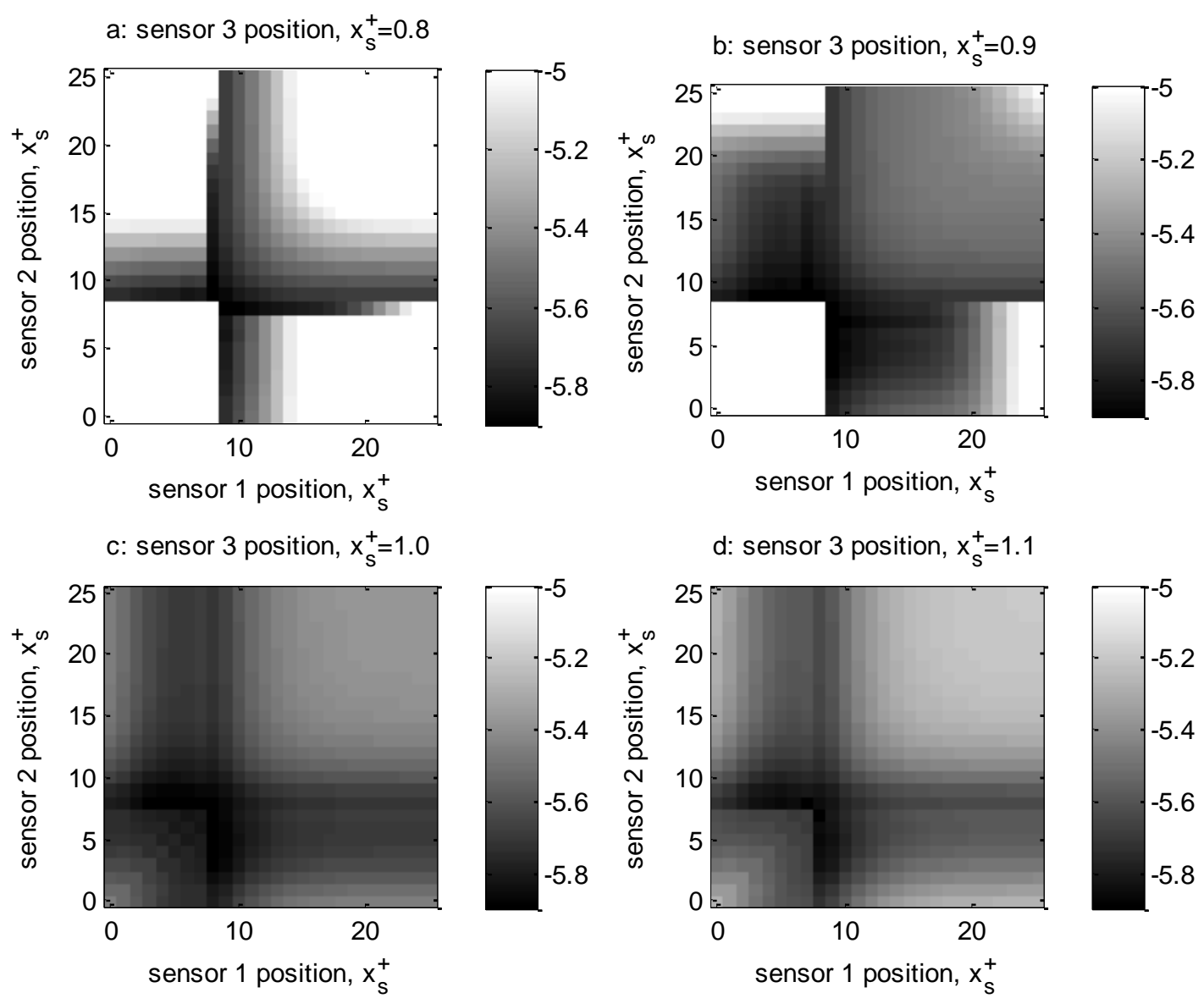

Figure 6.15: Contact distribution RMS residual plots for various sensor locations.

These data all shows trends similar to the two sensor data, but with mildly lower residuals. This shows that each sensor is still helping to reduce error in the estimation. However, it is obvious that the biggest single factor is the placement of the first sensor. The other sensors help only somewhat. This third sensor is exhibiting significant diminishing returns on the estimation quality. That said, it still exhibits the observed preference for each measurement being stacked on the trailing nodes of the contact. This result is suspected to be a result of having added no error to the simulated data for this case. 


\subsubsection{D, 1B Cases}

Now the cases to be covered are the two dimensional cases. These cases will give a better understanding of how the moving solid behaves in depth. These cases are built upon the understanding developed in the one dimensional cases. This formulation continues to assume that all the heat enters the moving body, but removes the assumption that the material is thin enough to assume roughly uniform temperatures in the ' $y$ ' direction. The non-dimensional formulation is presented above in Table 6.11. In addition to the required parameters in the 1D, 1B study, the 2D, 1B study requires the that the thickness $L_{y}^{+}$be specified.

\section{Numerical Resolution Case}

This first case again looks at the behavior of the direct model to ensure that the numerical method gives physical results that are reasonably converged. Again, with regard to the application of this paper, an exact numerical convergence is not required. The physical parameters used to generate the data are provided in Table 6.21.

Table 6.21: Physical parameters for the 2D numerical resolution study.

\begin{tabular}{|c|c|}
\hline \multicolumn{2}{|c|}{ Physical Parameters } \\
\hline$L_{x}^{+}$ & 10 \\
\hline$L_{y}^{+}$ & 0.1 \\
\hline$S t$ & 0.1 \\
\hline$L_{y}^{+}\left(x^{+}\right)$ & $\begin{cases}1 & x^{+} \leq 1 \\
0 & x^{+}>1\end{cases}$ \\
\hline
\end{tabular}

With the use of these parameters, the temperature solution can be determined. These results are presented below in Figure 6.16. The first plot in the figure shows the solution with 100 nodes in the ' $y$ ' direction at $t^{+}=25$. The second plot shows the solution with 200 nodes in the ' $\mathrm{y}$ ' direction, again at $t^{+}=25$. Both of these cases retain the use of 100 nodes in the ' $\mathrm{x}$ ' direction from the previous one dimensional cases. These plots, qualitatively demonstrate very similar behaviors. 


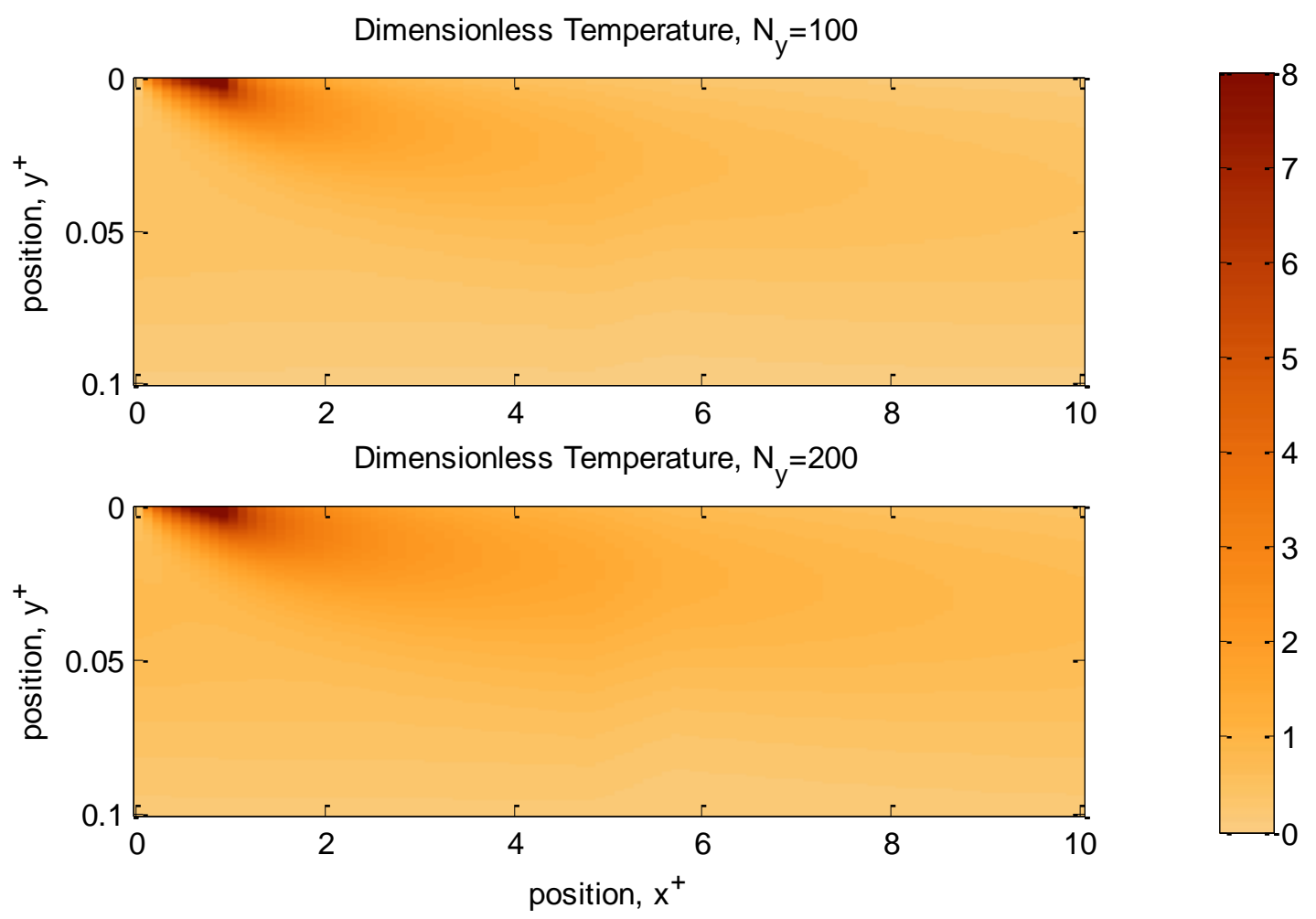

Figure 6.16: Comparison of dimensionless temperature using 100 and 200 ' $y$ ' nodes.

This figure shows some very interesting behavior. As a result of allowing the conductive mechanism, one can see that immediately downstream of the source the warmest part of the body is actually subsurface. This result would indicate that for some systems, it may be beneficial to measure the temperature downstream of the source and slightly subsurface. With regard to the numerical convergence of the solution, there is no immediately visible difference in the solution between the two graphs above. Thus, for the purposes of this study, 100 nodes in both the ' $x$ ' and ' $y$ ' directions for the $2 \mathrm{D}$ case is sufficiently converged.

\section{Varied ' $y$ ' Direction measurement location case}

This case study discusses the effect of the depth of the sensor on the measurement quality. This study is important because when the sensor is placed deeper into the material, the data becomes far less sensitive to the contact. This is because the only means by which energy is transferred to the deeper material is through conduction. Conduction, by and large, is far slower than bulk transport. Also, since it is diffusive in nature, the data becomes less sharp. Thus, it is useful to know how deep one can place the measurement location before the data is useless. The physical and measurement parameters as they were used for this study are below in Table 6.22. 
Table 6.22: Parameters for the varied ' $y$ ' measurement location.

\begin{tabular}{|c|c|}
\hline \multicolumn{2}{|c|}{ Physical Parameters } \\
\hline$L_{x}^{+}$ & 10 \\
\hline$L_{y}^{+}$ & 1 \\
\hline$S t$ & 1 \\
\hline$L_{y}^{+}$ & \\
\hline$f_{d}^{+}\left(x^{+}\right)$ & $\begin{cases}1 & x^{+} \leq 1 \\
0 & x^{+}>1\end{cases}$ \\
\hline \multicolumn{2}{|c|}{ Measurement Parameters } \\
\hline$N_{s}$ & 1 \\
\hline$x_{s}^{+}$ & {$[0 \rightarrow 2.5]$} \\
\hline$y_{s}^{+}$ & {$[0 \rightarrow 0.05]$} \\
\hline$\pm \operatorname{err}^{+}($simulated $)$ & \pm 0.0 \\
\hline
\end{tabular}

As this case allows both the ' $x$ ' and ' $y$ ' positions of the sensor to move, one can now determine a field much like those determined above in the multiple sensors in 1D case. This field will give the preferred location of a single sensor in two dimensions. Further, it will give information to the effect of how deep one can place the sensor before there is no useable information. The RMS residual plot of the one sensor in two dimensions is shown below in Figure 6.17.
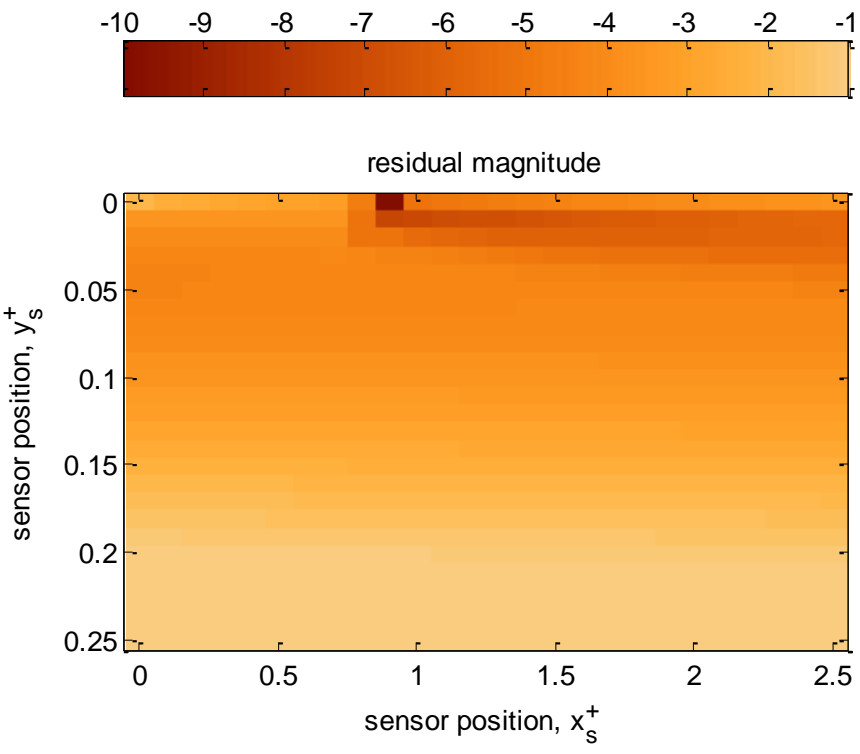

Figure 6.17: Contact distribution RMS residual plot for one sensor in two dimensions. 
With the relatively large Stanton number, the surface nodes, while providing the smallest residuals in the field, have a very limited zone where the results are good. However, these nodes fall off quickly, whereas the subsurface nodes, because they are not directly exposed to convection at the surface, retain their energy longer, and thus are more forgiving with regard to the location of the sensor in the ' $x$ ' direction.

\subsection{Two Body Problems}

The two body problems bring added physical realism and complexity to the problem. That said, the major issues and trends as presented in the one body problems do not change. As such, the addition of these new difficulties enables the application of the lessons learned above to extend the understanding of the more physically realistic situations. The major aspect that has now changed is that one must now account for the partition of heat. How one divides this is not a trivial matter, and was addressed in chapter 4.

\subsubsection{D, 2B Cases}

Now the case to be covered is the two body case. This case will give a better understanding of how the two solids interact. The system, as derived in chapter 3, is the 'pin-ondisk' arrangement. The non-dimensional formulation is presented above in Table 6.12. This implies that the nominal contact area is the whole extent of the static body. Thus, $L_{x_{1}}=L_{c o n}$, and thus we can continue to use $L_{x_{2}}=L_{x}$. These cases are built upon the understanding developed in the one dimensional, one body cases. Both bodies are examined and compared. The parameters used to generate the case are presented below in Table 6.23. 
Table 6.23: Parameters for the 1D, 2B sensor study.

\begin{tabular}{|c|c|}
\hline \multicolumn{2}{|c|}{ Physical Parameters } \\
\hline$L_{x}^{+}$ & 10 \\
\hline$\frac{S t}{L_{v_{2}}^{+}}$ & 0.5 \\
\hline$f_{d}^{+}\left(x^{+}\right)$ & $\begin{cases}1 & x^{+} \leq 1 \\
0 & x^{+}>1\end{cases}$ \\
\hline \multicolumn{2}{|c|}{ Measurement Parameters } \\
\hline$N_{s}$ & 1 \\
\hline$x_{s}^{+}$ & $\begin{cases}{[0 \rightarrow 1]} & \text { body } 1 \\
{[0 \rightarrow 10]} & \text { body } 2\end{cases}$ \\
\hline$\pm \operatorname{err}^{+}$(simulated) & \pm 0.0 \\
\hline
\end{tabular}

As this case allows the sensor position to be in either body, one can now compare the quality of the measurement in the static body with that of the moving body. This gives two RMS contact distribution residual traces. The two traces can be plotted together to give a comparison of their utility. The $\Delta f_{d_{R M S}}^{+}$plots of a single sensor in the different bodies are shown in Figure 6.18. The geometry of the system is shown directly above the plot in the figure. As a means of comparing the two residual plots, they are plotted against linear axes rather than semilogarithmic scales as done in the previous cases.

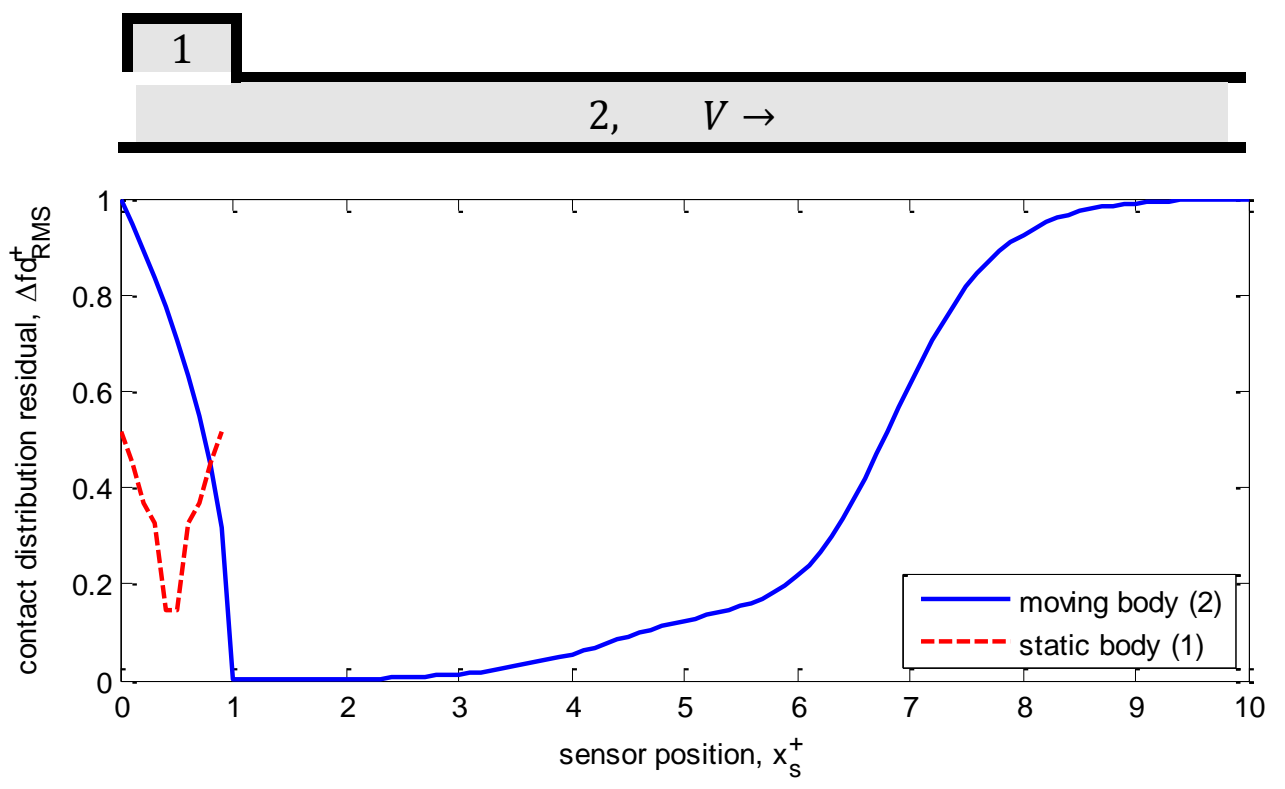

Figure 6.18: Contact distribution RMS residual plot for two bodies with geometry shown. 
These plots clearly demonstrate that the static body has a relatively poor capability to estimate the contact distribution compared to the moving body at its preferred location immediately downstream of the contact. However, the residuals do get reasonably small. This would seem to be because advection preserves the information more exactly. Further, if one looks at the sensitivity coefficients in the two regions, it shows that for the preferred nodes in either body, the node in the moving body sees the information from $x_{s}^{+}=0$ sooner than the static body. This is demonstrated in Figure 6.19.

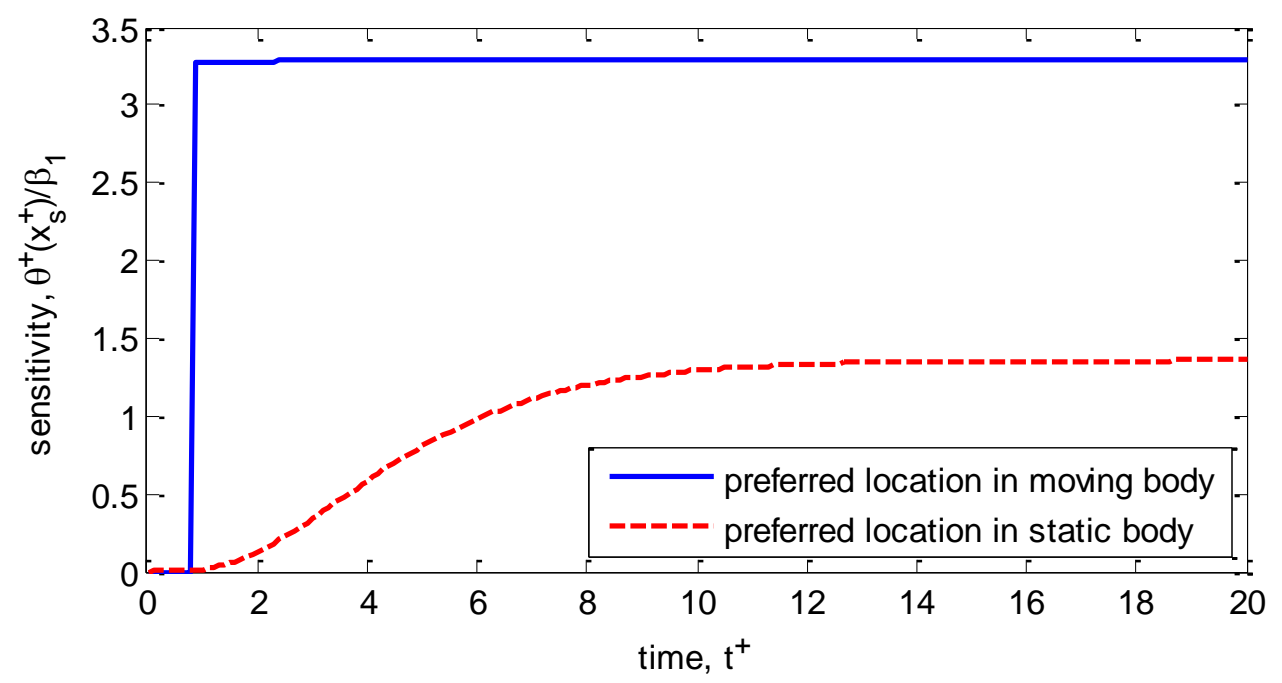

Figure 6.19: Sensitivity coefficients for each body at their preferred location. Each trace is for the parameter with which its communication is weakest.

The communication in the static body is slower, and thus the information is less well transmitted. Interestingly, this slow behavior was useful in the two dimensional case because the information is preserved as the body advects along. That allowed sensors to be placed much further away from the contact, as long as they were appropriately subsurface, thus making the measurement system more forgiving.

\subsubsection{D, 2B Case}

This case is a demonstration of the estimation algorithm with real material properties and stated dimensions. The non-dimensional formulation is presented above in Table 6.10. This is designed to show that the trends discovered above in the simpler models still apply and can be used in a more advanced situation. The parameters used in the estimation are stated in Table 
6.24. Of particular note is that, for this case a randomly generated contact distribution shows that the method can still estimate contact distributions in this complex case.

Table 6.24: Parameters for the demonstration of the 2D, 2B estimation.

\begin{tabular}{|c|c|}
\hline \multicolumn{2}{|c|}{ Physical Parameters } \\
\hline$h$ & $100 \frac{W}{m^{2} \cdot K}$ \\
\hline$T_{\infty}$ & $0^{\circ} \mathrm{C}$ \\
\hline$c_{C u}$ & $390 \frac{\mathrm{J}}{\mathrm{kg} \cdot \mathrm{K}}$ \\
\hline$\rho_{C u}$ & $8930 \frac{\mathrm{kg}}{\mathrm{m}^{3}}$ \\
\hline$k_{C u}$ & $400 \frac{W}{m \cdot K}$ \\
\hline$L_{x}$ & $1 \mathrm{~m}$ \\
\hline$L_{y_{1}}$ & $0.1 \mathrm{~m}$ \\
\hline$L_{y_{2}}$ & $0.1 \mathrm{~m}$ \\
\hline$q_{\text {fric }}$ & $1000000 \mathrm{~W}$ \\
\hline$V$ & $10 \frac{\mathrm{m}}{\mathrm{s}}$ \\
\hline$T_{0}$ & $0^{\circ} \mathrm{C}$ \\
\hline$L_{\text {con }}$ & $0.1 \mathrm{~m}$ \\
\hline \multicolumn{2}{|c|}{ Dimensionless Parameters } \\
\hline$P e$ & 8700 \\
\hline$S t$ & $2.9 \times 10^{-6}$ \\
\hline$L_{x}^{+}$ & 10 \\
\hline$L_{y_{2}}^{+}$ & 1 \\
\hline$f_{d}^{+}$ & randomly generated \\
\hline \multicolumn{2}{|c|}{ Measurement Parameters } \\
\hline$N_{S}$ & 1 \\
\hline$x_{S}$ & $14 \mathrm{~cm}$ \\
\hline$y_{s}$ & $3 \mathrm{~mm}$ \\
\hline err & $\pm 1^{\circ} \mathrm{C}$ \\
\hline
\end{tabular}

These parameters were selected such that it was assumed that the first millimeter of material was inaccessible for measurement (the first millimeter would be the top row of nodes) to simulate restricted access to the frictional surface. Thus, the measurements needed to be taken further downstream and not too deep, so as to have good sensitivity to the contact distribution parameters. Thus, the measurement was selected to be at a nodal position just a little under 5 centimeters downstream, and 3 millimeters deep. However, provided for comparison is the estimation with the sensor at the preferred measurement location. Both were simulated 
independently, and thus had differently generated contact distribution data. The estimated distributions are presented below in Figure 6.20.
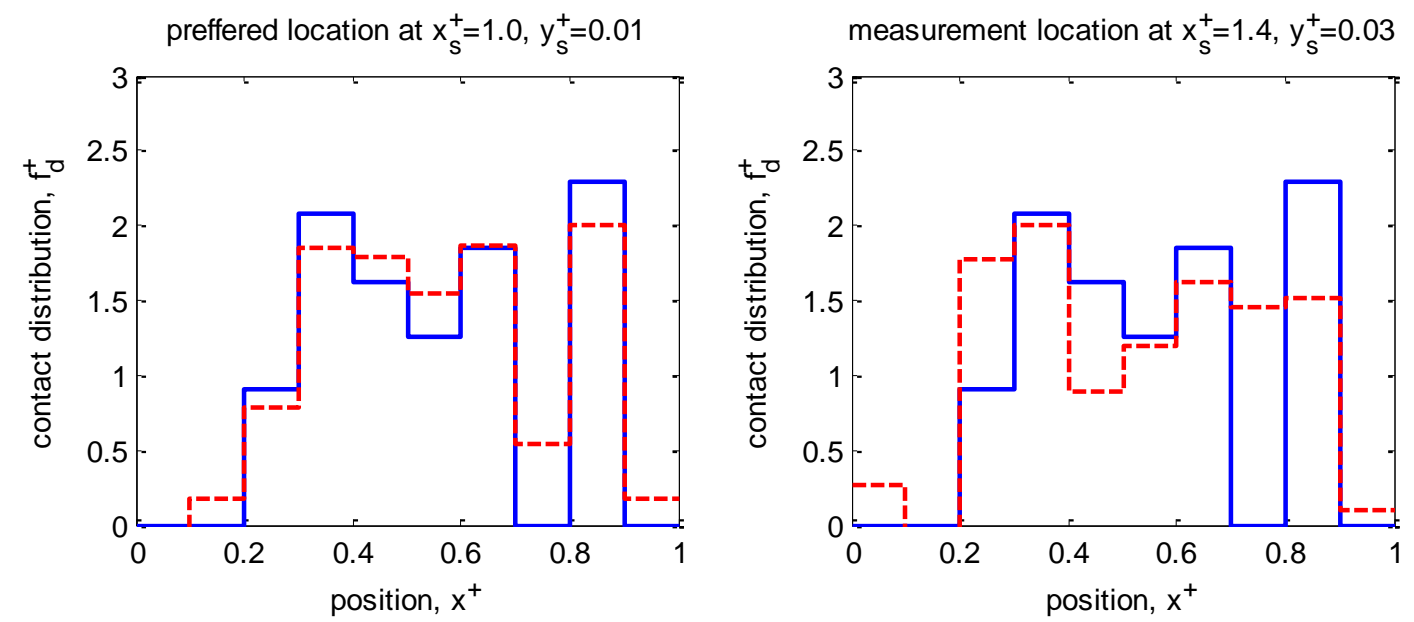

Figure 6.20: Actual and estimated contact distributions for the 2D, 2B demonstration. The solid line is the actual contact, and the dashed line is the estimated contact.

From this image, one sees that both measurement locations were able to reasonably back out the trends of the contact distribution. This success shows that the knowledge gained from the simpler models is very useful in selecting the measurement criteria in more complex models. This case helps validate the understanding gained from the simpler studies performed earlier. 


\section{Recommendations and Conclusions}

Here is a final summary of the work with final conclusions and recommendations for future work.

\subsection{Summary}

In summary, a means by which a time invariant real area of contact and surface temperature could be estimated using thermal measurements in $1 \mathrm{D}$ and $2 \mathrm{D}$ systems was developed. This technique utilized formulating a heat transfer model to simulate up to a two dimensional, two body sliding system. This estimation method was then used to provide experimental design guidance regarding measurement location for the estimation of the real contact area.

The thesis further covers the conversion of the above heat transfer model into a numerical, modified cellular automata technique. Once the rules for the modified cellular automata were developed, the inverse mathematics was covered to provide an understanding as to the method used to estimate the contact distribution. This method was then used on a variety of case studies to arrive at conclusions regarding the capabilities of this method to estimate the contact distribution.

\subsection{Conclusions}

This thesis has demonstrated that the preferred sensor location in the one and two dimensional systems does not change significantly when the contact distribution changes. Also, for the two dimensional estimations, the placement of the sensor was shown to be more forgiving if it was placed somewhat subsurface, as the information is preserved longer because the only transport mechanisms are conduction and advection. The addition of error to the data did not change the basic trends observed in the preferred location, but it did reduce the quality of the estimation of the contact distribution. Finally, it was shown that, while the moving body of the system was the preferred body to instrument, if necessary, the static body could be instrumented to achieve a reasonable approximation of the contact area.

This paper also utilized the two dimensional, two body case as an opportunity to demonstrate that the trends gleaned from simplified models can still be applied as the complexity increases. It showed that, through analysis of the residuals plots from simpler cases, information 
could be used to select the preferred measurement location, as well as acceptable measurement locations in the event that the preferred location is, for whatever reason, not accessible.

\subsection{Recommendations}

The success of the demonstration with the two dimensional, two body case indicates that the techniques used to develop these results shows promise and that there is significant future study that could be done that could provide useful insight. Of primary interest would be allowing the contact distribution to evolve in time. This investigation would significantly increase the number of parameters involved, but would model certain aspects of reality better. Secondly, and also of significant importance, would be an experimental validation of the modeling done within this paper. As this work was entirely computational, the question still remains about the full behavior in the real world. This work would likely best be done after the extension of this work into the time dependant contact distribution. Thirdly, and of particular interest to this analysis, would be the inclusion of further multi-physical modeling into the modified cellular automata method. This would imply the inclusion of physical processes such as chemical effects, mechanical vibrations, mechanics of solids, and electrical current flow through the contact. Each of these provides a potential benefit in terms of more accurate modeling of the system. These benefits add potential insight into the contact distribution.

While the above mentioned recommendations are probably the most important, there is further follow on work that could provide interesting information as well. These include the extension of the model into three dimensions, studying the effect of property ratios, and having multiple sensors in the higher dimensional systems. These all grant further capability to estimate the contact distribution. In particular, with regard to more experimental applications, the physical effects of thee dimensional systems or dissimilar materials can have great implications on the thermal field evolved in the solid. The addition of additional sensors in two or three dimensional systems can provide a great informational boon to the experimenter.

To conclude, this work has provided interesting insights to and developed trends regarding the estimation of the contact distribution in sliding systems using temperature measurements. It has further laid a ground work for a large body of immediate follow on work that could prove fruitful. 


\section{List of References}

1. Surface Temperatures and Tribological Behavior of Pure Metallic Elements. Vick, B., M. Furey, and K. Iskandar. Brisbane : s.n., 1998. AUSTRIB '98.

2. Vick, B. Image Collection. Blacksburg, 2009.

3. Coalescence and Breakup of Contact Areas: Effects of Surface Temperatures. Furey, M. J., B. Vick, H. M. R. Ghasemi and J. H. Bohn. 2007, Tribology International, Vol. 40, pp. 595-600.

4. Temperature of Rubbing Surfaces. Archard, J. 1959, Wear, pp. 438-455.

5. Flash Temperature Concept. Blok, H. 1963, Wear., pp. 483-494.

6. Possible Flash Temperatures in Slider and Recording Disk Transient Contact. Ettles, C. 1986, ASLE Transactions., pp. 321-328.

7. Tribochemistry and Tribopolymerization. Furey, M. J., and C. Kajdas, R. Kempinski, G.J. Molina, and B. Vick. Atlanta : s.n., 2006. 231st American Chemical Society National Meeting.

8. Moving Sources of Heat and Temperature at Sliding Contacts. Jaeger, J. 1942, Royal Society of New South Wales -- Journal and Proceedings., pp. 203-224.

9. Thermal and Thermomechanical Effects in Dry Sliding. Kennedy, F. 1984, Wear., pp. 453476.

10. Probable Interface Temperatures of Solids in Sliding Contact. Ling, F., and S. Pu. 1964, Wear., pp. 23-36.

11. Maximum and Average Flash Temperatures in Sliding Contacts. Tian, X. and F. Kennedy. 1994, Journal of Tribology, pp. 167-174.

12. Visualization of Temperatures in Sliding Contact. Vick, B. and M. Furey. Vienna : s.n., 2001. 2nd World Tribology Conference.

13. A Basic Theoretical Study of the Temperature Rise in Sliding Contact with Multiple Contacts.

Vick, B. and M. J. Furey. 2001, Tribology International, Vol. 34, pp. 823-829.

14. Thermal Analysis of Sliding Contact in Systems With Rotary Motion. Vick, B., and Furey. Washington, D.C. : s.n., 2005. Proceedings of WTC2005, World Tribology Conference III. 
15. Modeling of Contact Area, Contact Force, and Contact stiffness of Mechanical Systems with Friction. Abdo, J and Shamseldin, E. s.1. : ASME, 2005. 2005 ASME International Mechanical Engineering Congress and Exposition. pp. 295-302.

16. The Area of Contact between Stationary and Between Moving Surfaces. Bowden, F., and D.

Tabor. s.l. : The Royal Society, 1939. Proceedings of the Royal SOciety of London.

Series A, Mathematical and Physical Sciences. pp. 391-413.

17. Contact of Friction Surfaces in Clutches. Gekker, F. and Fedorov., and A. 1984, Soviet Journal of Friction and Wear., pp. 79-86.

18. Estimation of the Number and the Mean Area of Real Contact Points on the Basis of Surface Profles. Kimura, Y. 1969, Wear, pp. 47-55.

19. Estimation of the Thermal Properties and Interface Conditions of Heterogeneous Materials.

Loulou, T., E. P. Scott, and B. Vick. New Orleans : s.n., 2002. Proceedings of the A.S.M.E., IMECE'02.

20. Real Contact Area of Fractal-Regular Sufraces and Its Implications in the Law of Friction.

Wang, S. 2004, Journal of Tribology v. 126.

21. Inverse estimation of heat flux and temperature in multi-layer gun barrel. Tsung-Chien

Chen, Liu, Chiun-Chien, Jang, H.-Y. and Tuan, Pan-Chio. June 2007, International Journal of Heat and Mass Transfer, v 50, n 11-12, pp. 2060-2068.

22. Contact Analysis of Elastic-Plastic Fractal Surfaces. Yan, W. and K. Komvopoulos. 1998, Journal of Applied Physics, pp. 3617-3624.

23. Inverse heat transfer analysis of grinding, part 1: Methods. Guo, C. (Univ of

Massachusetts, Amherst, United States) and Malkin, S. 1996, Journal of engineering for industry, v 118, n 1, pp. 137-142.

24. Inverse heat transfer analysis of grinding, part 2: Applications. Guo, C. (Univ of

Massachusetts, Amherst, United States) and S. Malkin. 1996, Journal of engineering for industry,v 118, pp. 143-149.

25. Three-dimensional inverse heat transfer analysis during the grinding process. Wang, C.-C.

(Dept. of Mech. Eng., Nat. Cheng Kung Univ., Tainan, Taiwan) and Chen, C.-K. s.l. : Journal of Mechanical Engineering Science, 2002. Proceedings of the Institution of Mechanical Engineers, Part C. pp. 199-212. 
26. Method for Determination of Local Heat Flux from Transient Temperature Measurements.

Powell, W. and T. Price. 1964, Instrument Society of America, pp. 246-254.

27. Özisik, M., and H. Orlande. Inverse Heat Transfer: Fundamentals and Applications. New York : Taylor \& Francis, 2000.

28. Beck, J., B. BlackWell, and C. St. Clair. Inverse Heat Conduction: Ill-posed Problems. s.l. : John Wiley \& Sons, Inc., 1985.

29. On the Solution of the Inverse Heat Conduction Problem by Finite Differences. Farifo, L., V. Schrock, and E. Spedicato. 1975, Energia Nucleare, pp. 452-464.

30. The Accuracy and Resolving Power of One Dimensional Transient Inverse Heat Conduction Theory as Applied to Discrete and Inaccurate Measurements. Hills, R. and G.

Mulholland. 1979, Journal of Heat and Mass Transfer, pp. 1221-1229.

31. Optimal regularizaton methods for inverse heat transfer problems. Okamoto, Kei (School of Mechanical and Materials Engineering, Washington State University, Pullman, WA 99164) and Li, Ben Q. 2004. Proceedings of the ASME Heat Transfer/Fluids Engineering Summer Conference. pp. 825-834.

32. Methodology for Comparison of Inverse Heat Conduction Methods. Raynaud, M. and J. Beck. 1985, Journal of Heat Transfer, pp. 30-37.

33. The Inverse Problem in Transient Heat Conduction. Sparrow, E., A. Haji-Sheikh, and T. Lundgren. 1964, Journal of Applied Mechanics, pp. 369-375.

34. Combined parameter and function estimation with application to thermal conductivity and surface heat flux. Loulou, Tahar. October 2007, Journal of Heat Transfer, v 129, n 10, pp. 1309-1320.

35. Analysis and Solution of the Ill-Posed Inverse Heat Conduction Problem. Weber, C. 1981, Journal of Heat and Mass Transfer, pp. 1783-1792.

36. Incropera, F., D. DeWitt, T. Bergman, A. Lavine. Fundamentals of Heat and Mass Transfer, sixth ed. s.l. : John Wiley \& Sons, 2007.

37. Vick, B. Applied Mathematics: A Visual Approach. Course Notes for ME 5304: Heat Conduction. Blacksburg : Virginia Tech, 2008.

38. Thermal Analysis of Solids at High Peclet Numbers Subjected to Moving Heat Sources. Manca, O., B. Morrone, and S. Nardini. 1999, Journal of Heat Transfer, pp. 182-186. 
39. Multi-Physics Modeling Using Cellular Automata. Vick, B. 2007, Complex Systems, Vol. 17 (1 \& 2), pp. 65-78. 


\section{Appendix}

\section{Parameter Estimation Program:}

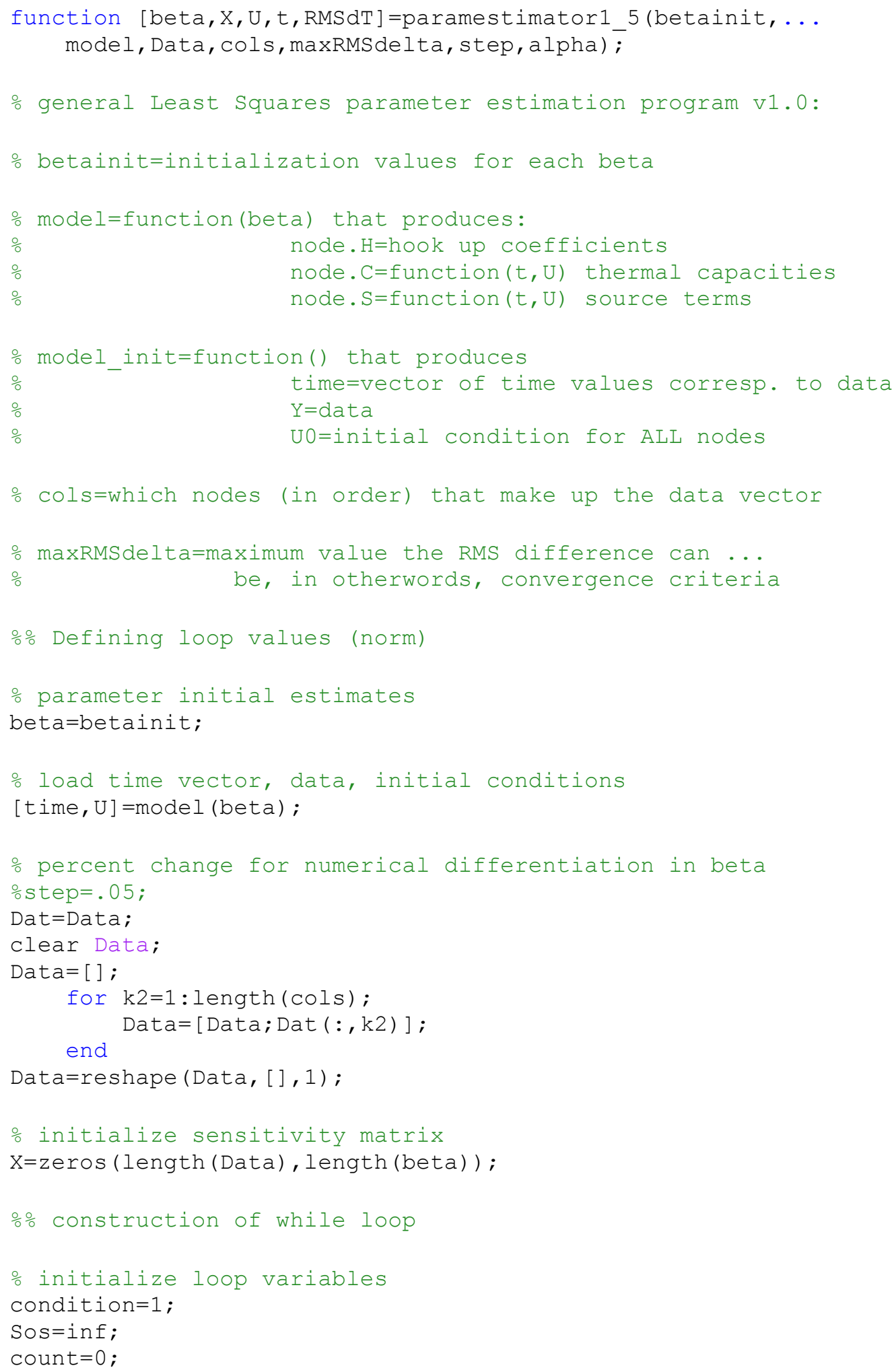




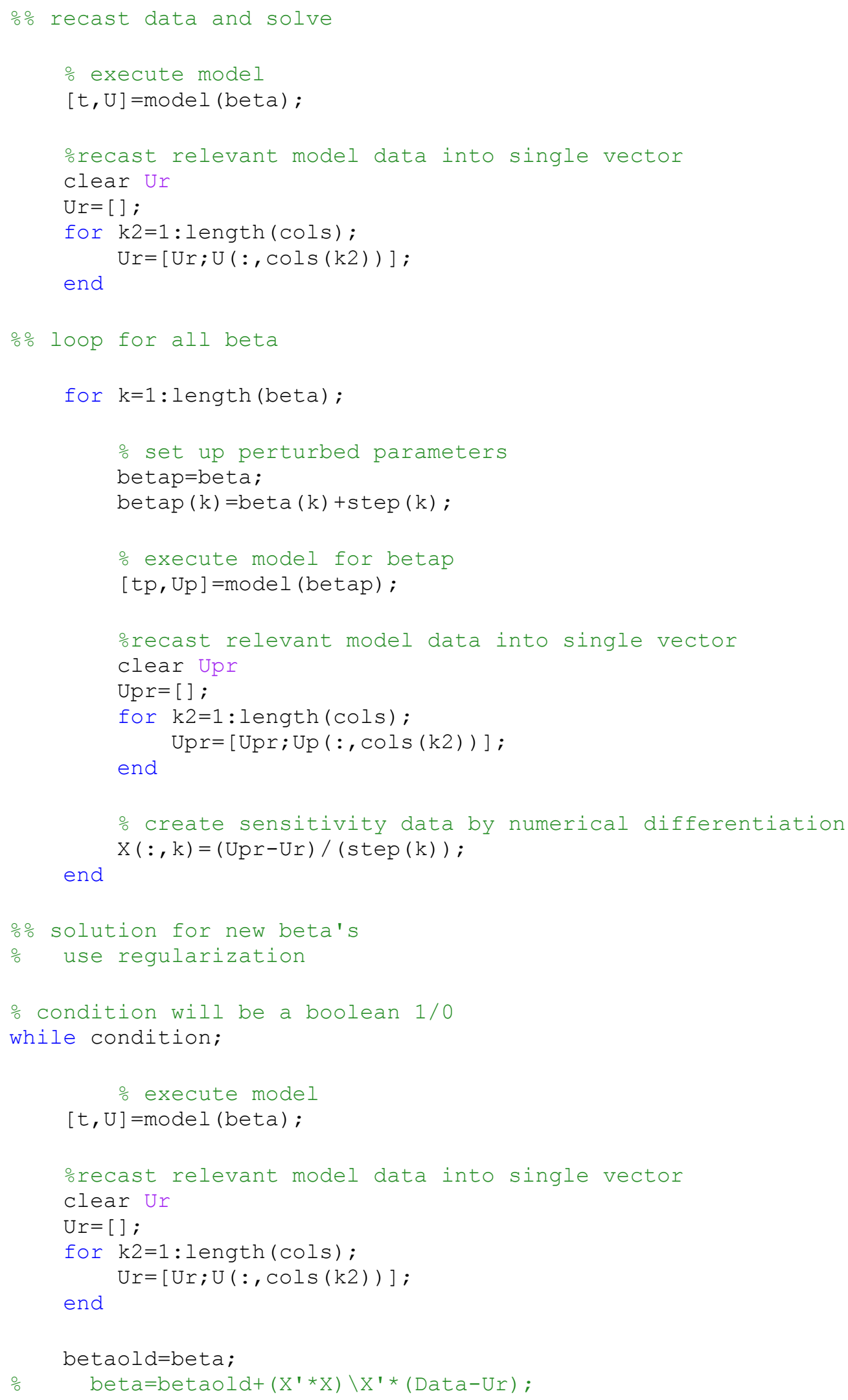




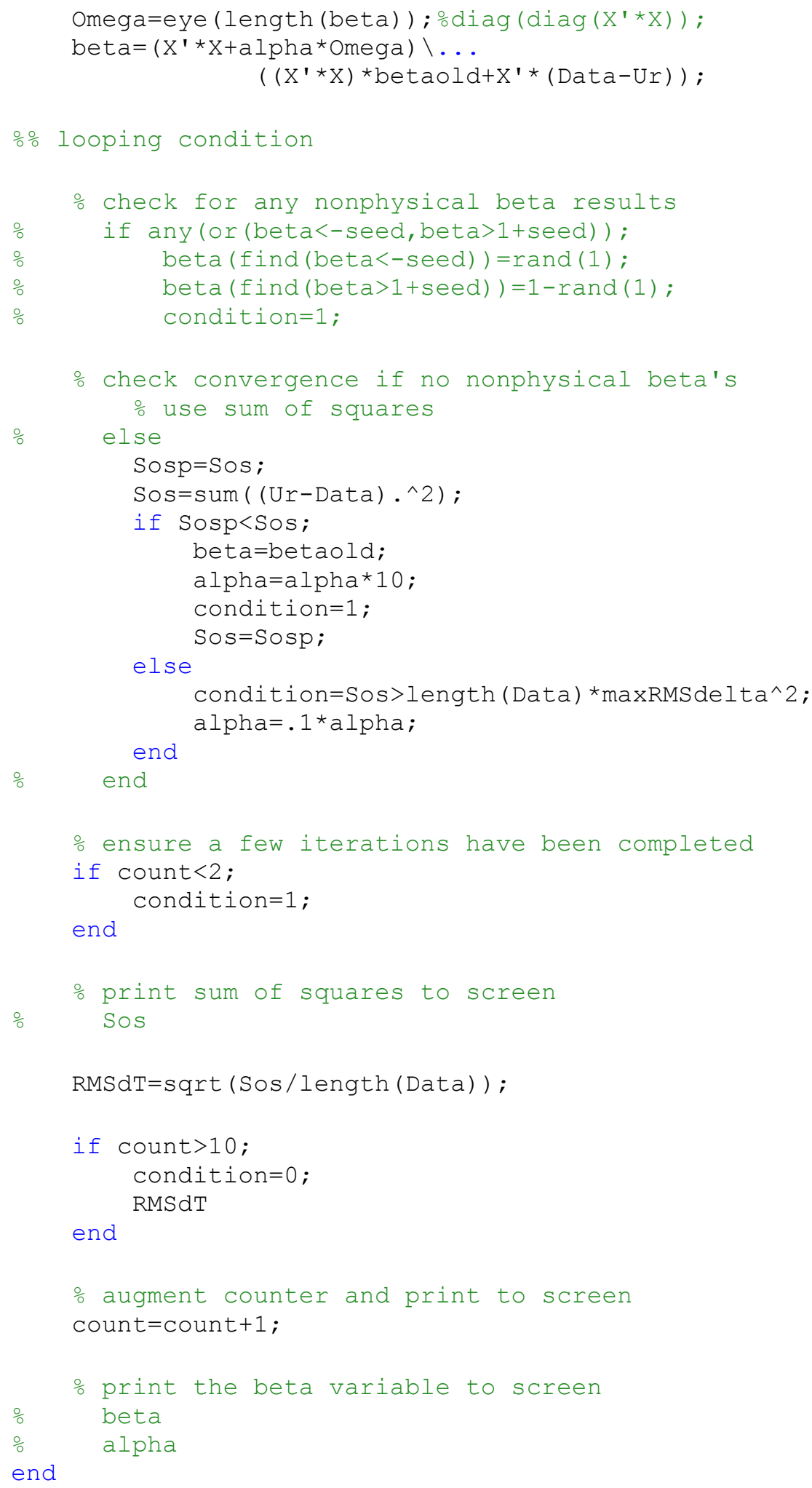




\section{D, 1B Direct Model:}

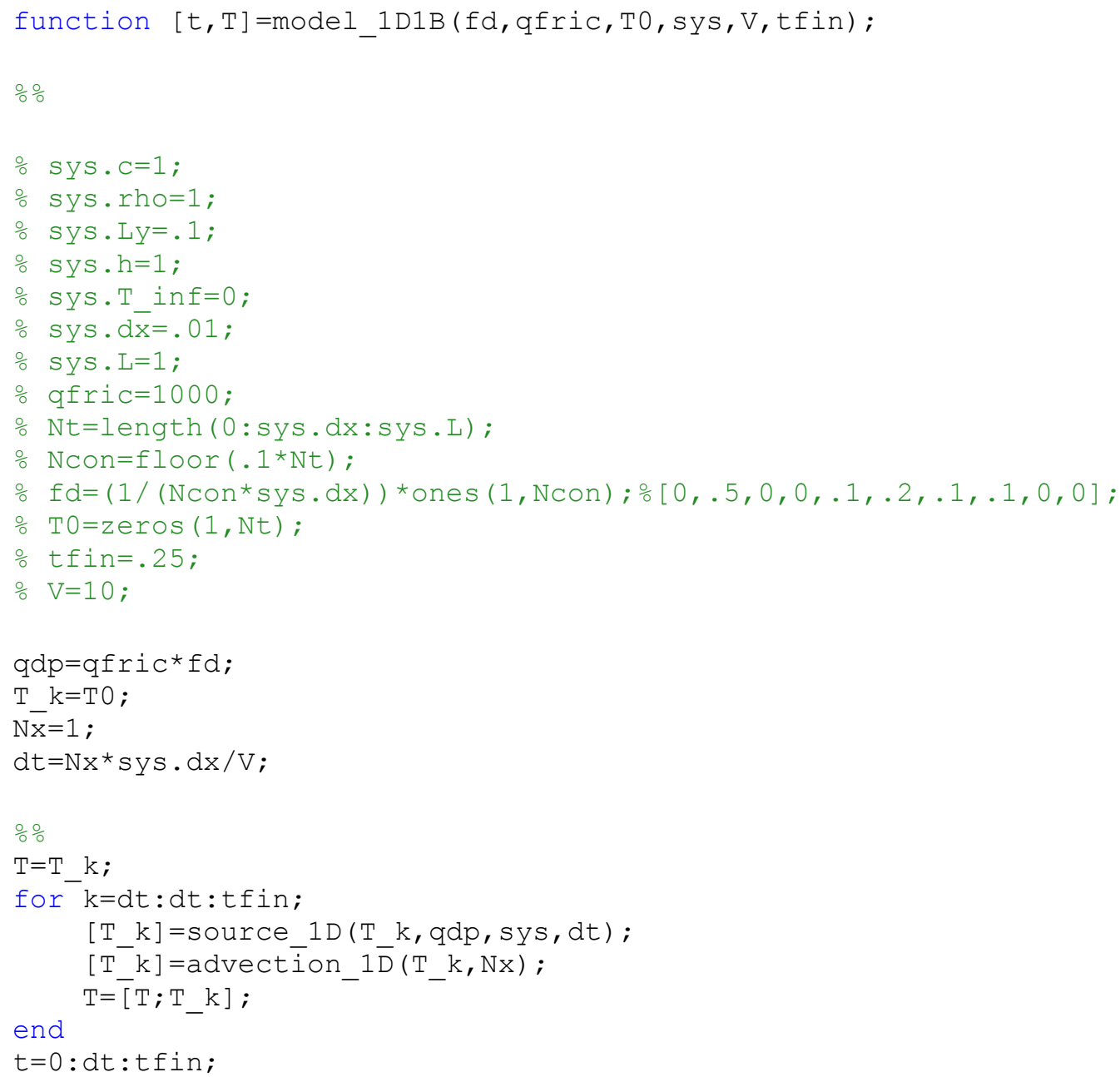

\section{D, 1B Direct Model Driver:}

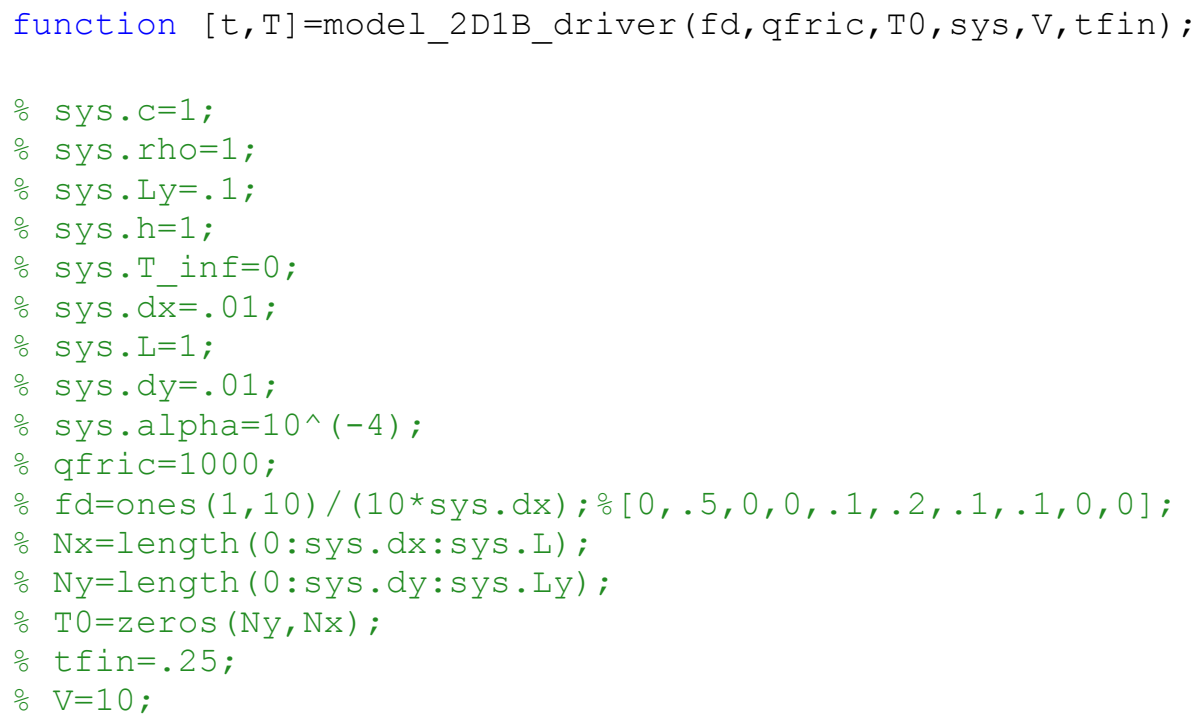




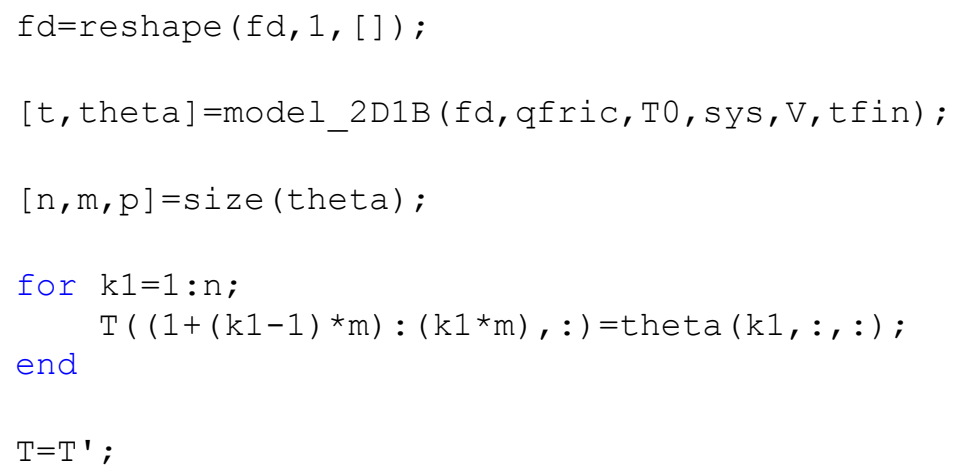

\section{D, 1B Direct Model:}

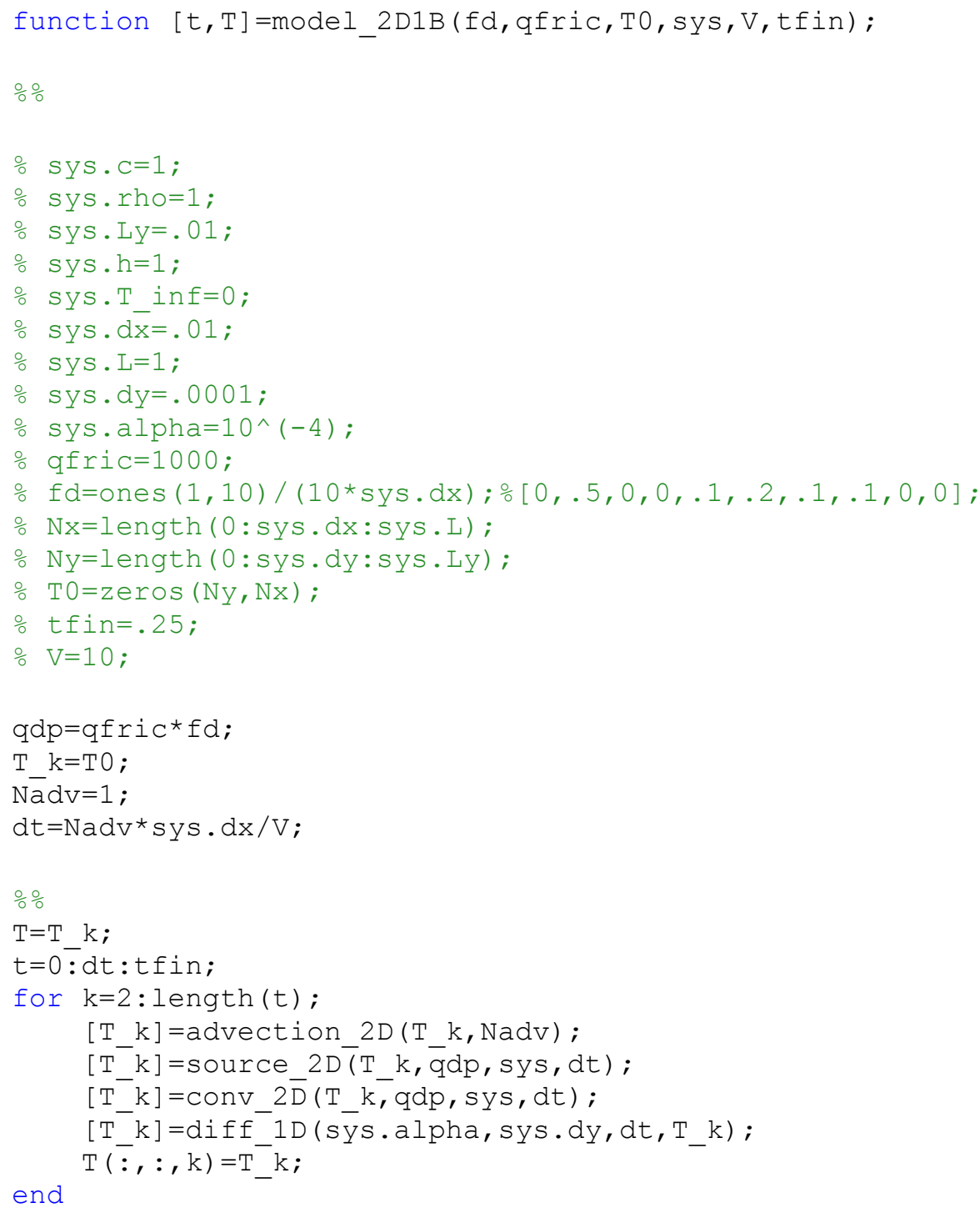




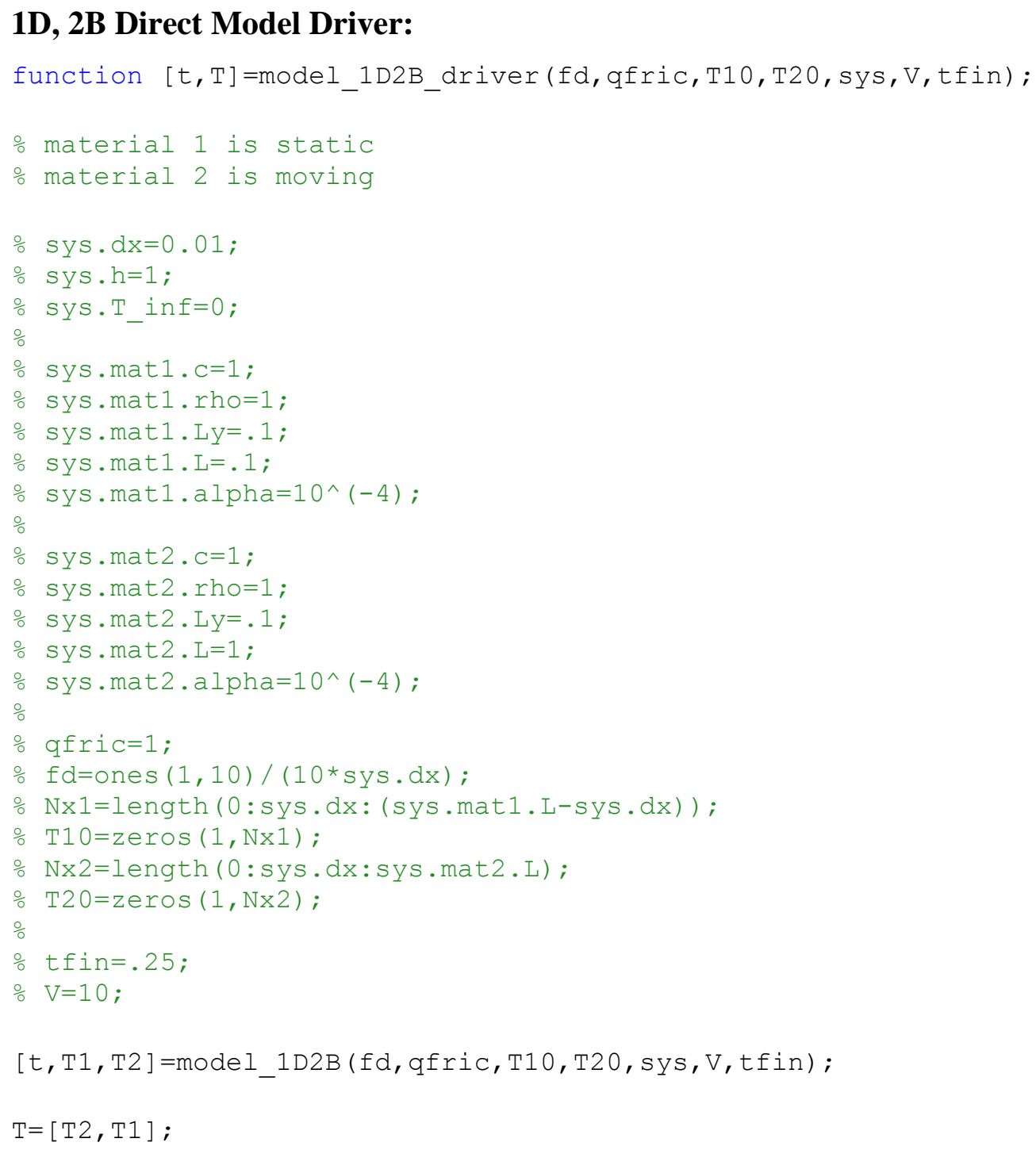

\section{D, 2B Direct Model:}

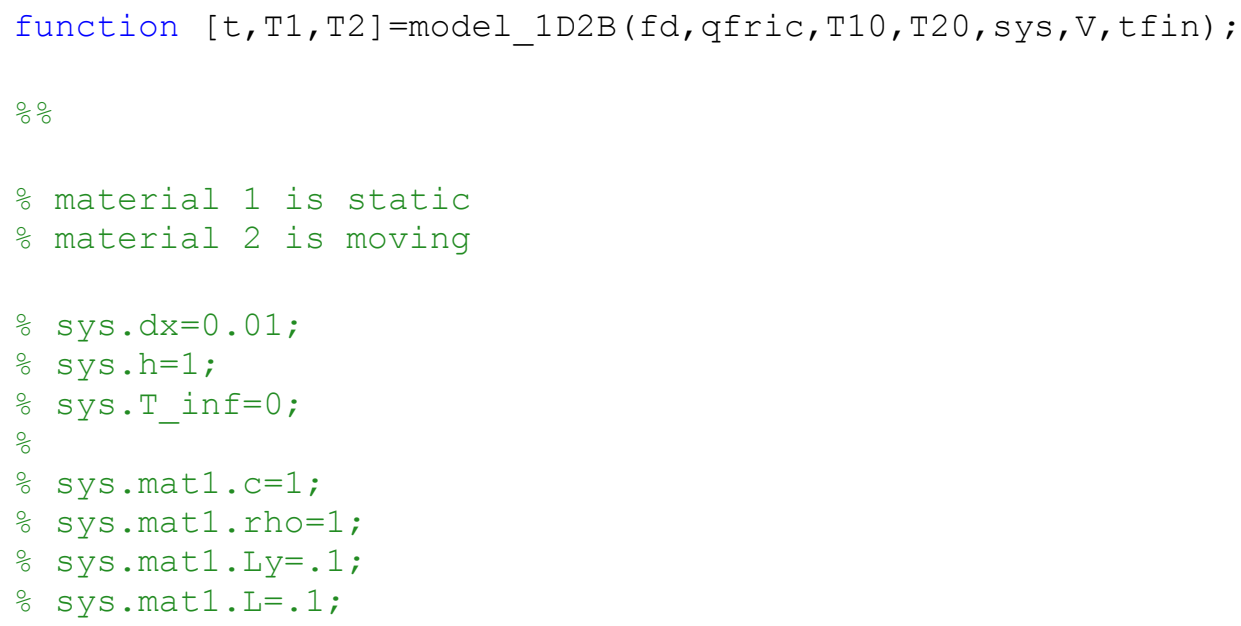




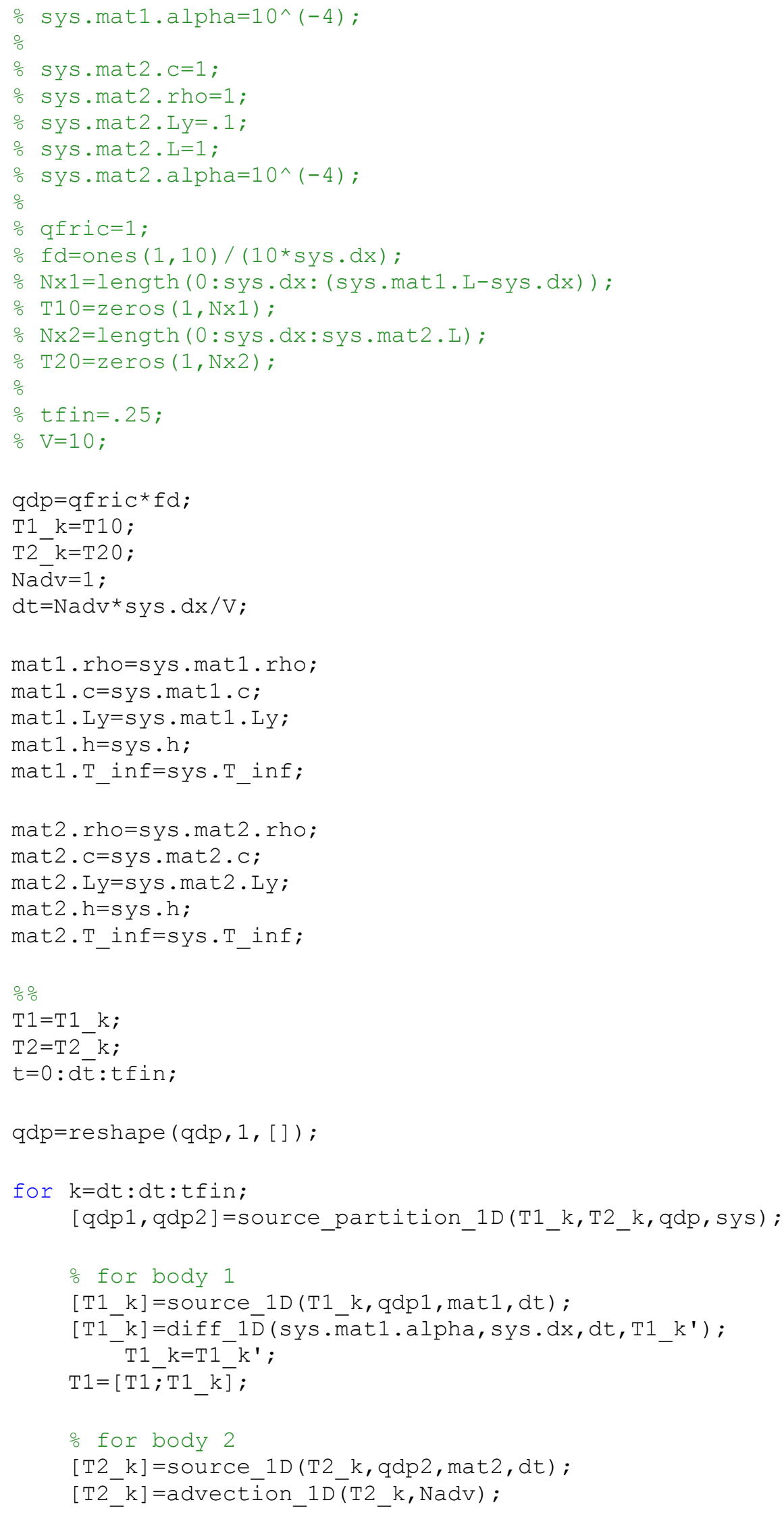


end

$\mathrm{T} 2=[\mathrm{T} 2 ; \mathrm{T} 2 \ldots \mathrm{k}]$

\section{D, 2B Direct Model Driver:}

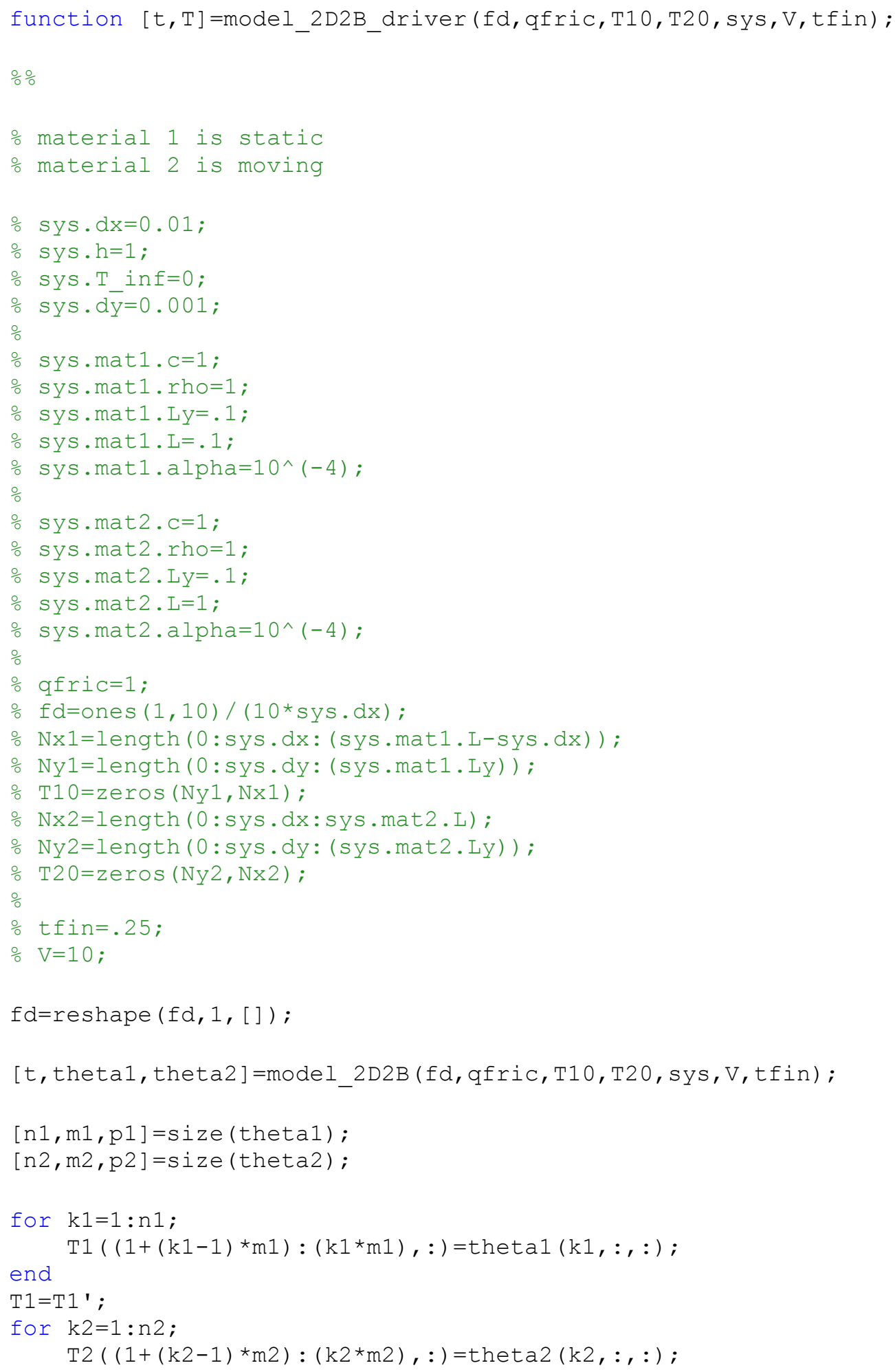


end

$\mathrm{T} 2=\mathrm{T}^{\prime}{ }^{\prime} ;$

$\mathrm{T}=[\mathrm{T} 2, \mathrm{~T} 1]$

\section{D, 2B Direct Model:}

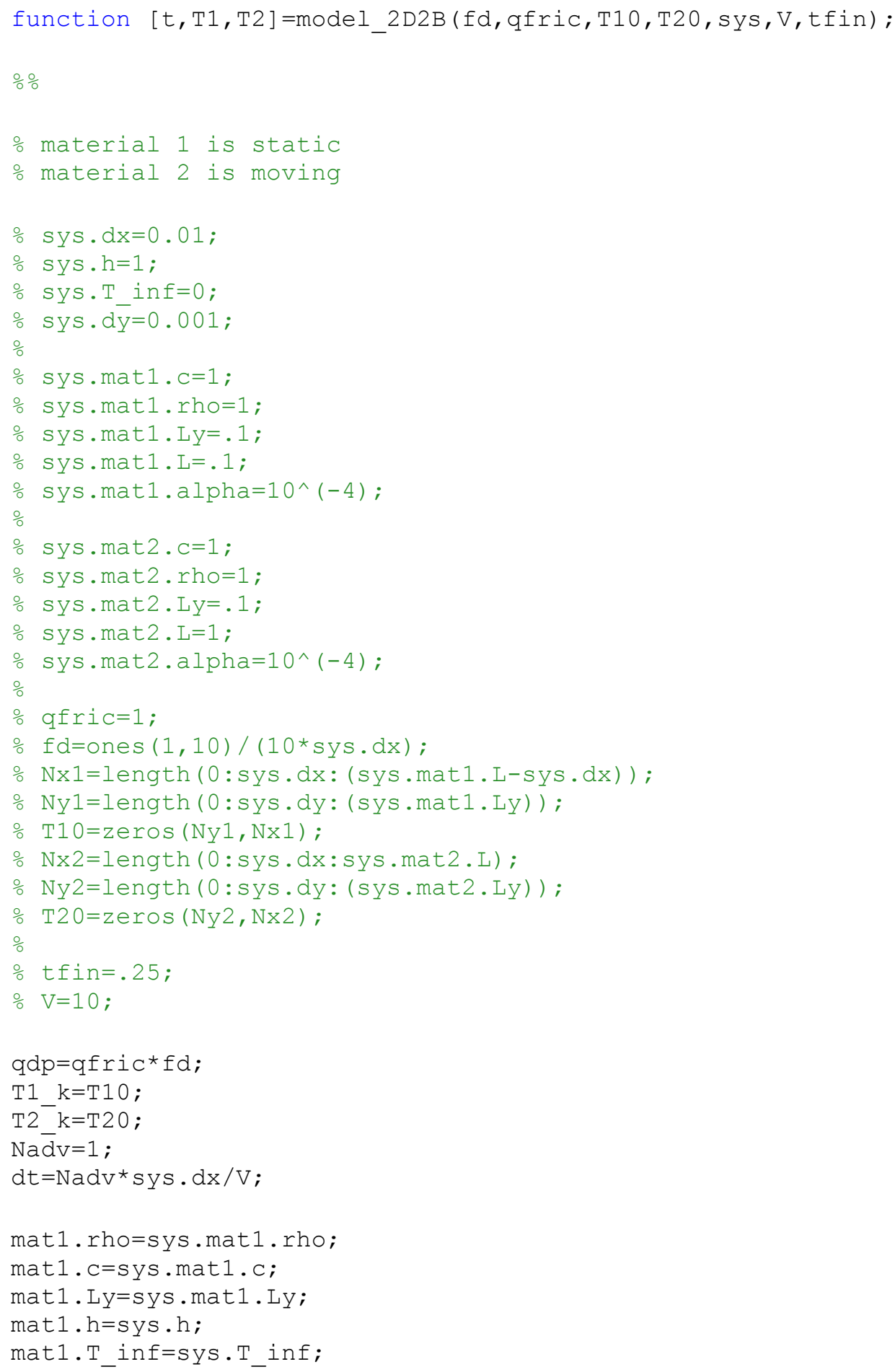




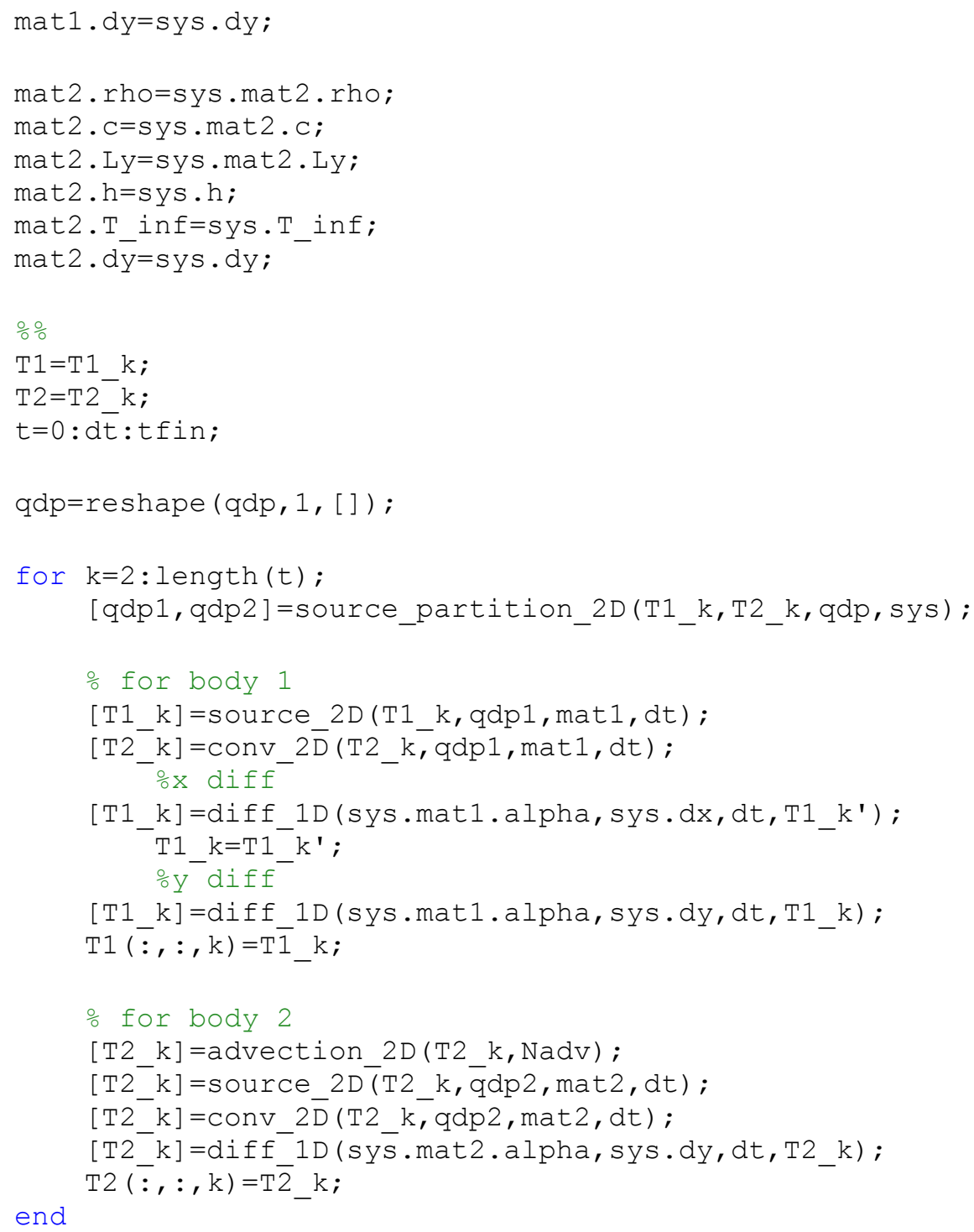

\section{Surface Temperature:}

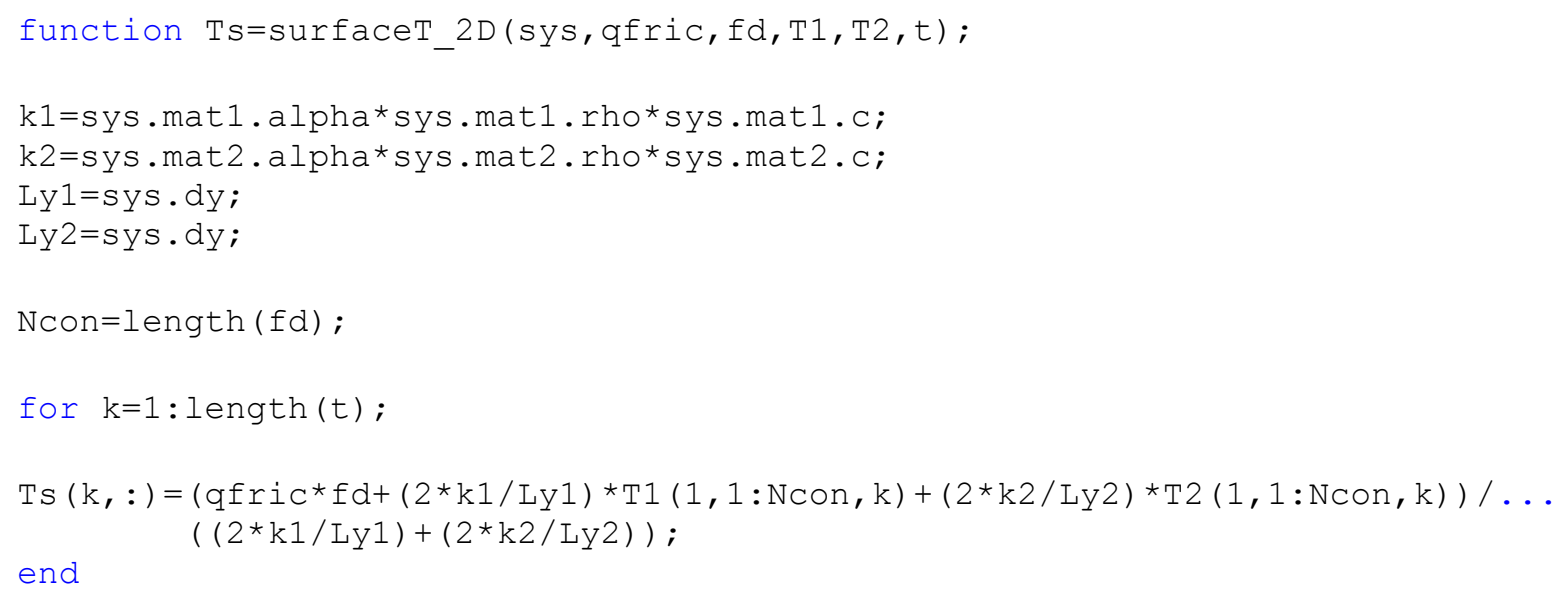




\section{D Advection Rule:}

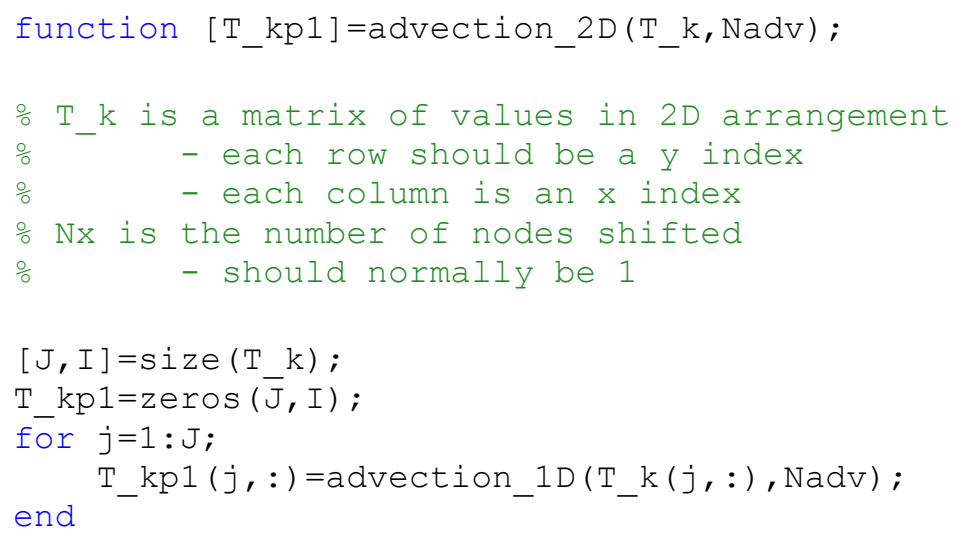

\section{D Advection Rule:}

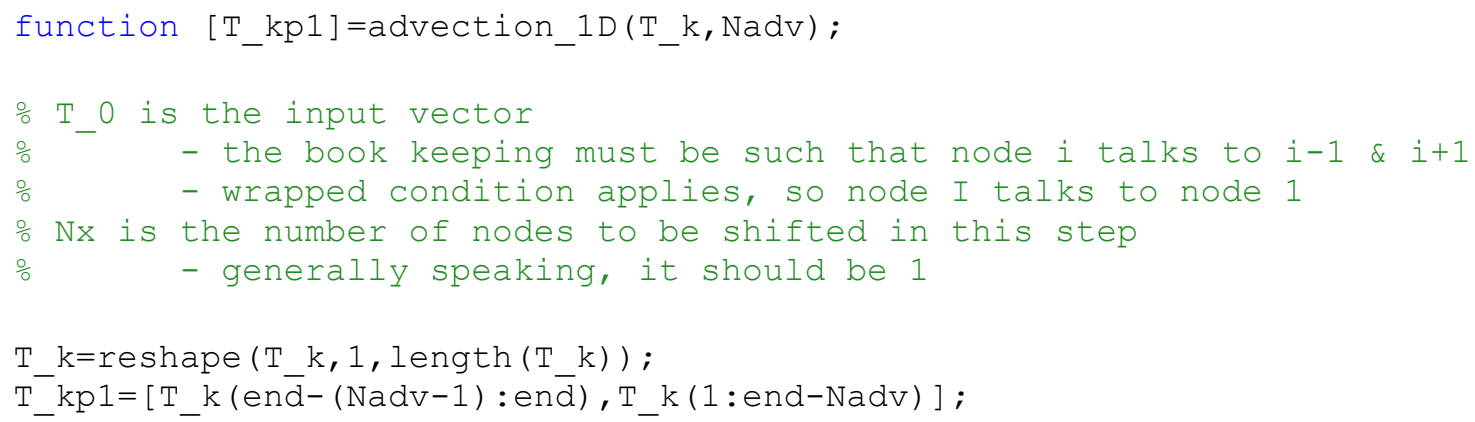

\section{Diffusion Rule:}

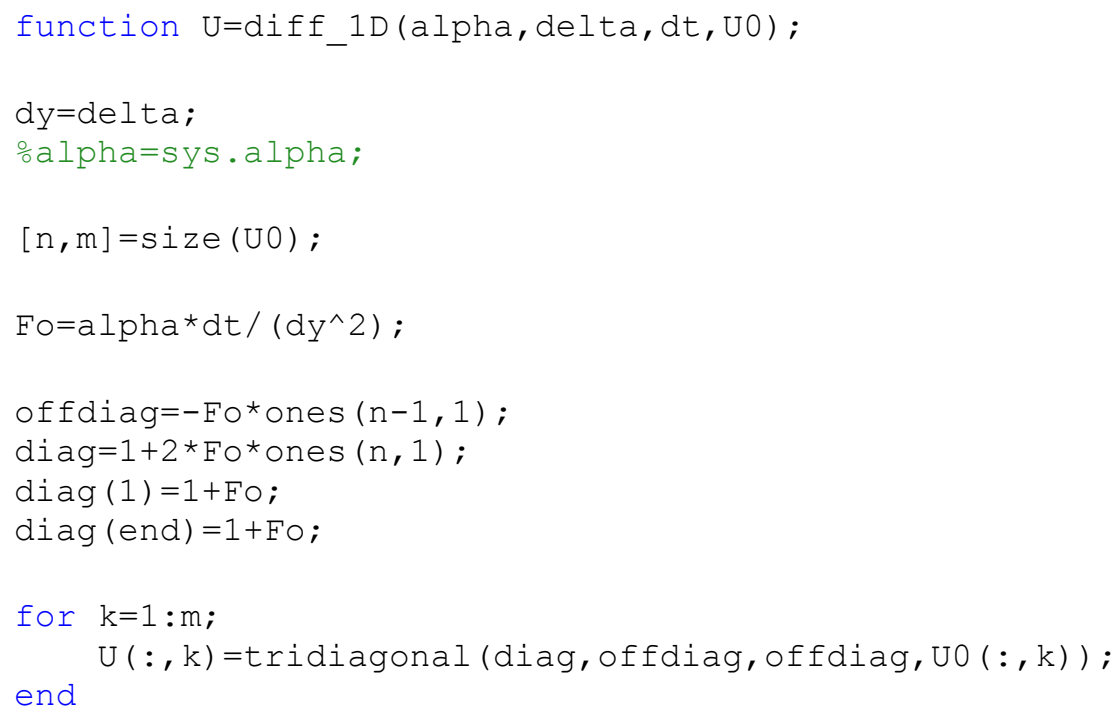




\section{Tridiagonal Solver (source uncertain):}

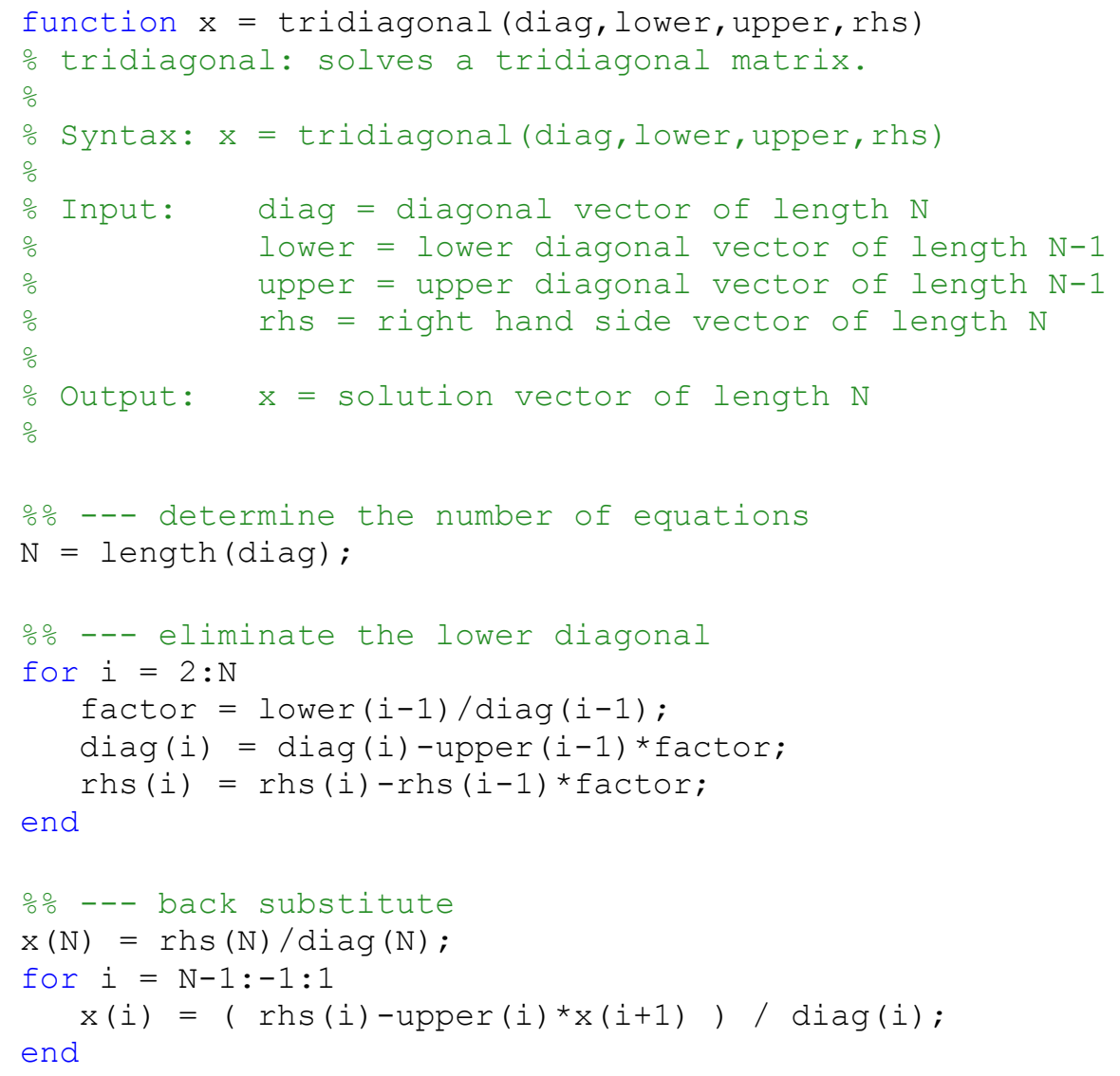

\section{D Source:}

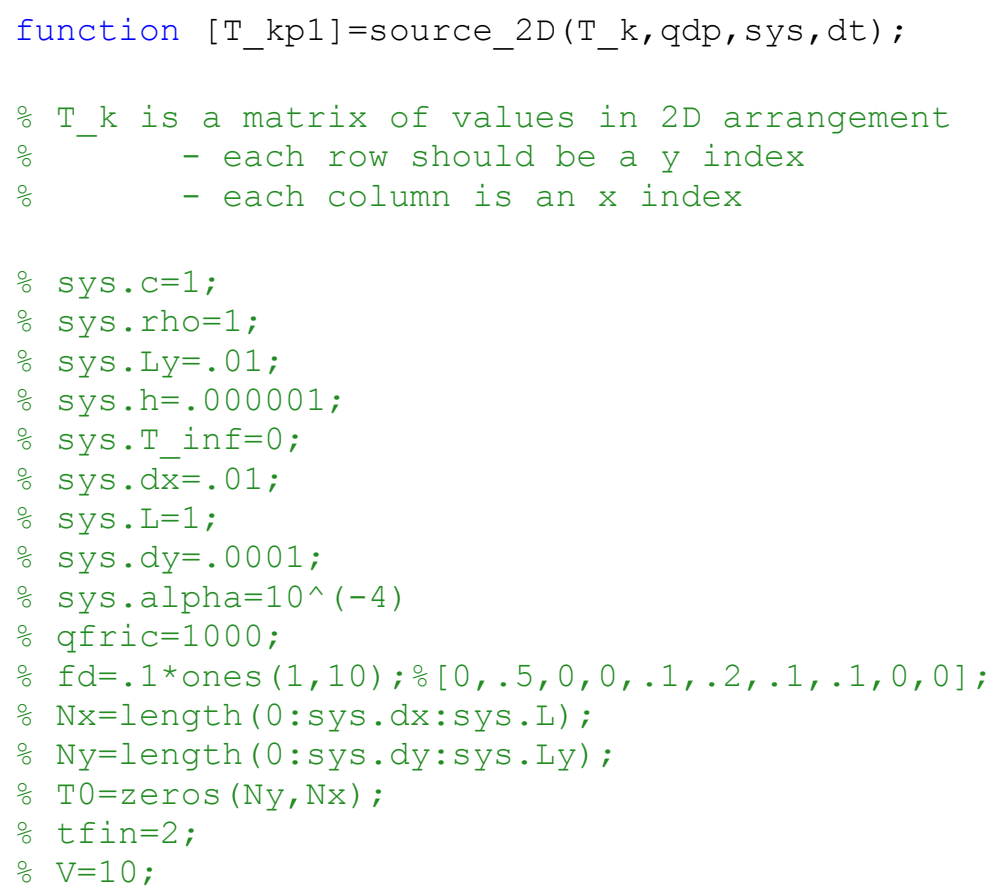


Ncon=length $(q d p)$;

$\mathrm{T}_{-} \mathrm{kpl}=\mathrm{T}_{-} \mathrm{k}$;

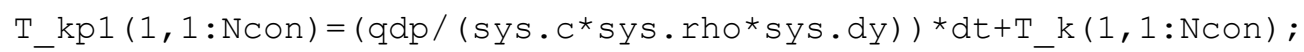

\section{D Source:}

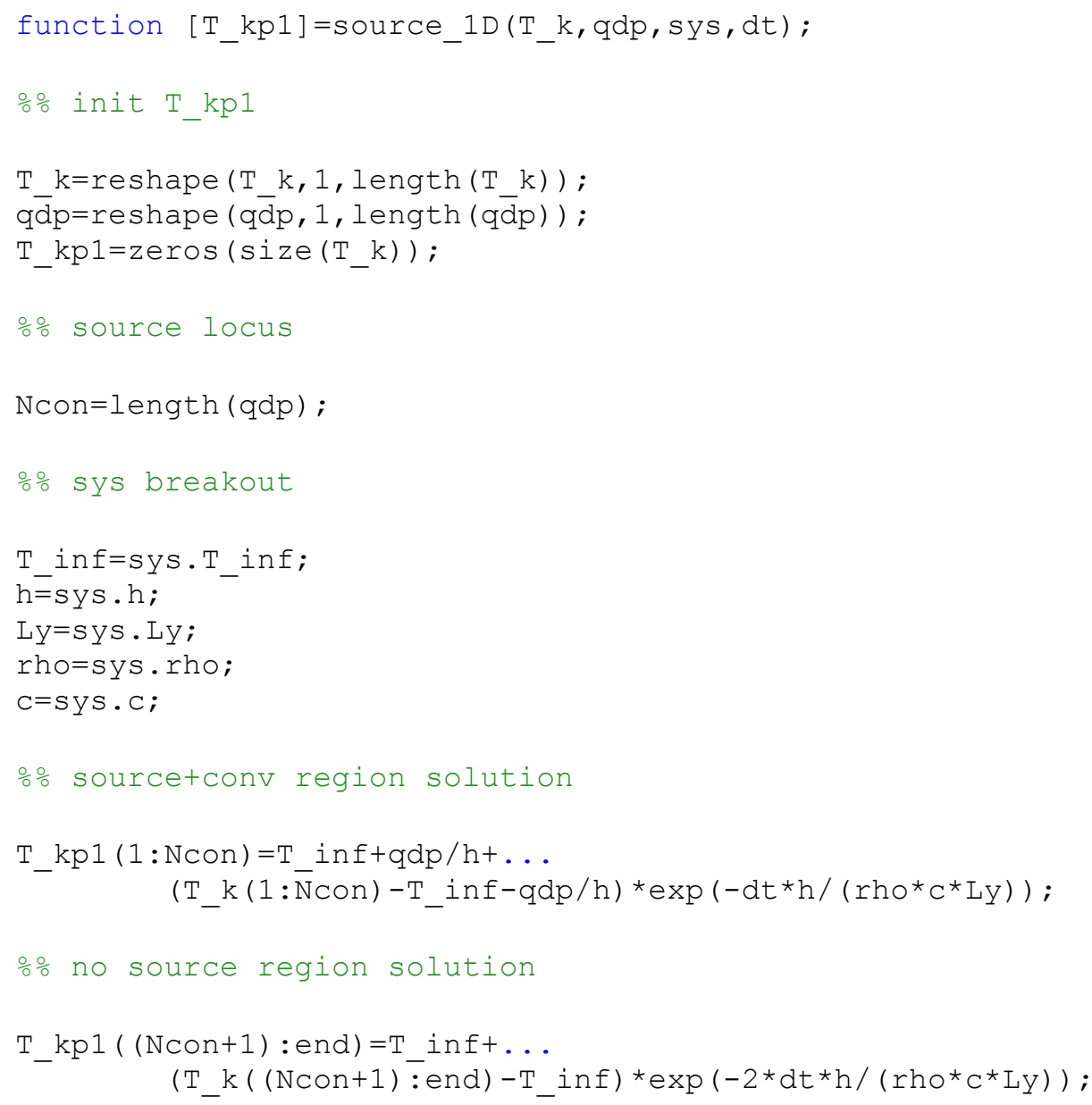

\section{D Convection:}

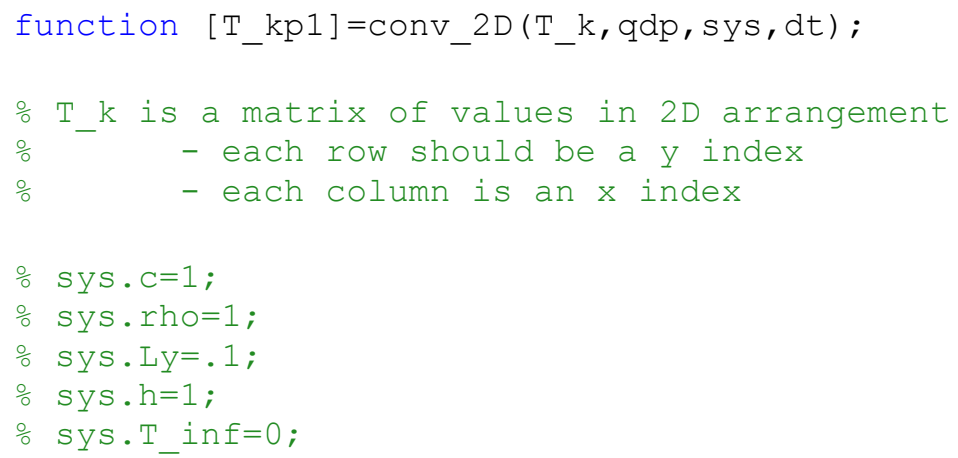




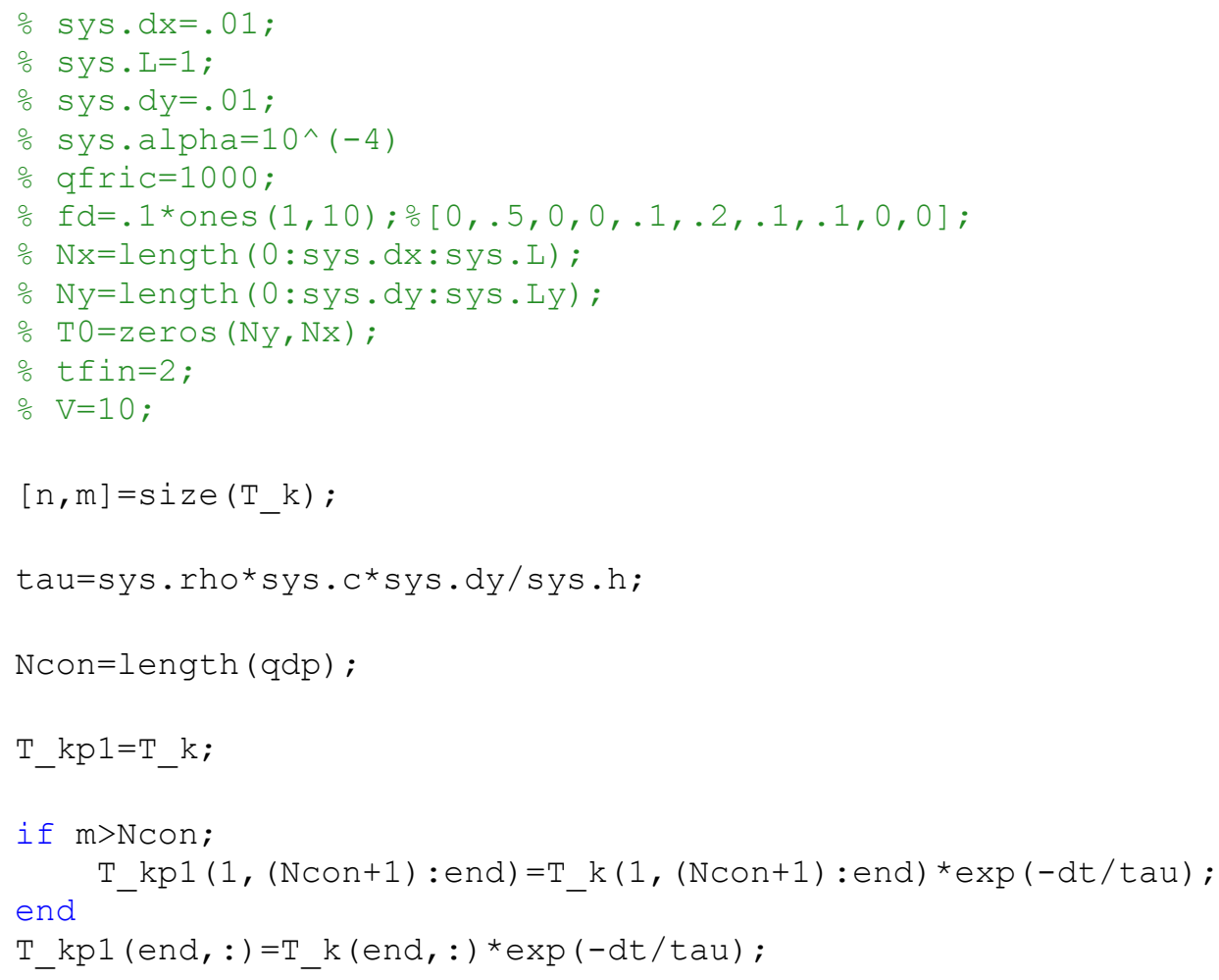

\section{D Source Partition:}

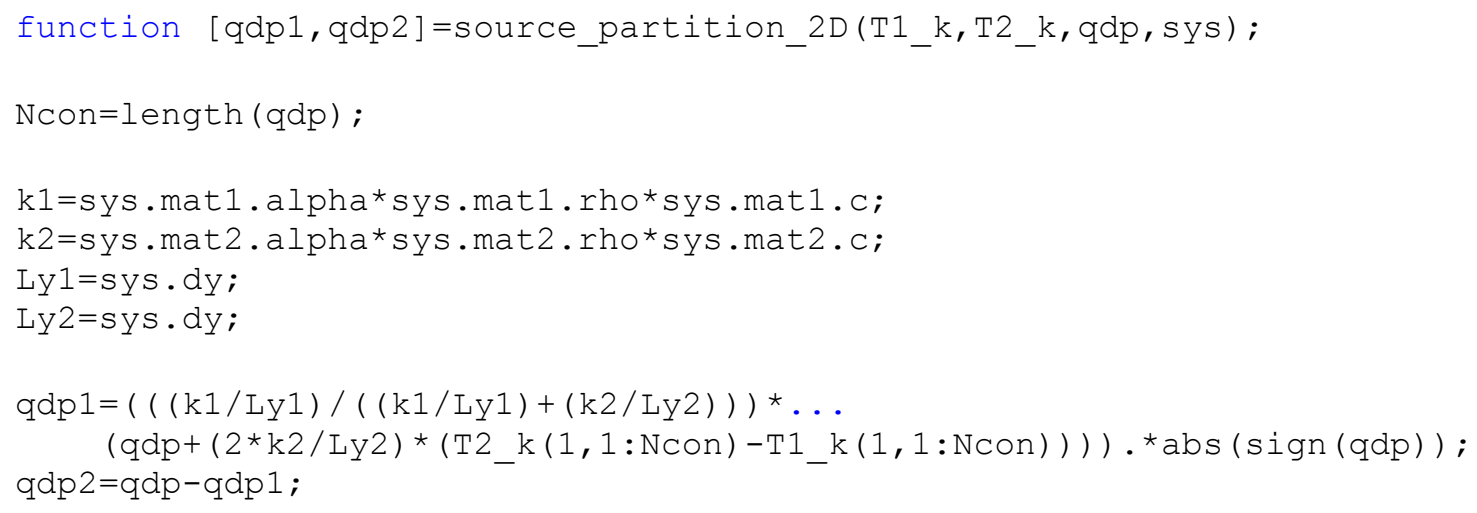

\section{D Source Partition:}

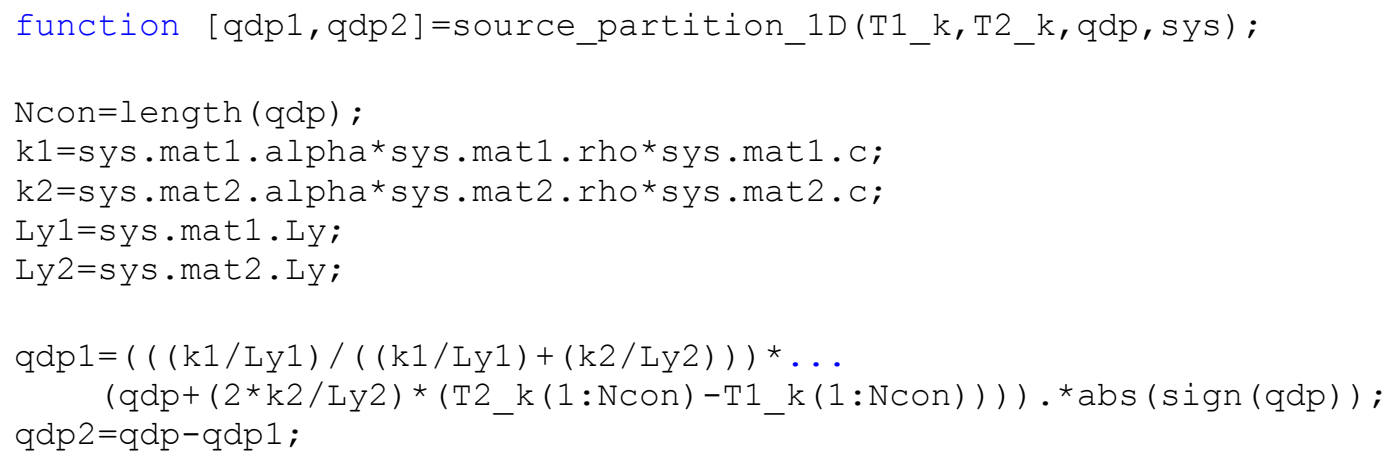

